\title{
The circular law for sparse non-Hermitian matrices
}

\author{
Anirban Basak* and Mark Rudelson ${ }^{\dagger}$ \\ Department of Mathematics \\ Weizmann Institute of Science \\ POB 26, Rehovot 76100, Israel \\ e-mail: anirban.basak@weizmann.ac.il \\ Department of Mathematics \\ University of Michigan \\ East Hall, 530 Church Street \\ Ann Arbor, Michigan 48109, USA \\ e-mail: rudelson@umich.edu
}

\begin{abstract}
For a class of sparse random matrices of the form $A_{n}=\left(\xi_{i, j} \delta_{i, j}\right)_{i, j=1}^{n}$, where $\left\{\xi_{i, j}\right\}$ are i.i.d. centered sub-Gaussian random variables of unit variance, and $\left\{\delta_{i, j}\right\}$ are i.i.d. Bernoulli random variables taking value 1 with probability $p_{n}$, we prove that the empirical spectral distribution of $A_{n} / \sqrt{n p_{n}}$ converges weakly to the circular law, in probability, for all $p_{n}$ such that $p_{n}=$ $\omega\left(\log ^{2} n / n\right)$. Additionally if $p_{n}$ satisfies the inequality $n p_{n}>\exp (c \sqrt{\log n})$ for some constant $c$, then the above convergence is shown to hold almost surely. The key to this is a new bound on the smallest singular value of complex shifts of real valued sparse random matrices. The circular law limit also extends to the adjacency matrix of a directed Erdős-Rényi graph with edge connectivity probability $p_{n}$.
\end{abstract}

MSC 2010 subject classifications: 15B52, 60B10, 60B20.

Keywords and phrases: Random matrix, sparse matrix, smallest singular value, circular law.

\section{Introduction}

For a $n \times n$ matrix $B_{n}$ let $\lambda_{1}\left(B_{n}\right), \lambda_{2}\left(B_{n}\right), \ldots, \lambda_{n}\left(B_{n}\right)$ be its eigenvalues. The empirical spectral distribution (ESD) of $B_{n}$, denoted hereafter by $L_{B_{n}}$, is given by

$$
L_{B_{n}}:=\frac{1}{n} \sum_{i=1}^{n} \delta_{\lambda_{i}}
$$

where $\delta_{x}$ is the dirac measure at $x$. If $B_{n}$ is a non-normal matrix (i.e. $B_{n} B_{n}^{*} \neq$ $B_{n}^{*} B_{n}$ ) then its eigenvalues are complex valued, resulting in $L_{B_{n}}$ being supported on the complex plane. Furthermore, when $B_{n}$ is random its ESD is a random probability measure. Thus, to study the asymptotics of ESDS of random matrices one needs to define appropriate notions of convergence. This can be done in one

\footnotetext{
${ }^{*}$ Research partially supported by grant 147/15 from the Israel Science Foundation.

${ }^{\dagger}$ Research partially supported by NSF grant DMS 1464514.
} 
of the two following ways: If $\left\{\mu_{n}\right\}$ is a sequence of random probability measures such that for every $f \in C_{b}(\mathbb{C})$, i.e. $f: \mathbb{C} \mapsto \mathbb{R}$ is bounded, $\int_{\mathbb{C}} f d \mu_{n} \rightarrow \int_{\mathbb{C}} f d \mu$ in probability, for some probability measure $\mu$ (possibly random), then $\mu_{n}$ is said to converge weakly to $\mu$, in probability. If $\int_{\mathbb{C}} f d \mu_{n} \rightarrow \int_{\mathbb{C}} f d \mu$ almost surely, then $\mu_{n}$ is said to converge to $\mu$ weakly, almost surely.

The study of the ESD for random matrices can be traced back to Wigner $[45,46]$ who showed that the ESD's of $n \times n$ Hermitian matrices with i.i.d. centered entries of variance $1 / n$ (modulo symmetry) satisfying appropriate moment bounds (for example, Gaussian) converge to the semicircle distribution. The conditions on the finiteness of the moments were relaxed in subsequent works, see $[6,34]$ and the references therein.

The rigorous study of non-Hermitian matrices, in particular non-normal matrices, emerged much later. The main difficulties were the sensitivity of the eigenvalues of non-normal matrices under small perturbations and the lack of appropriate tools. For example, Wigner's proof employed the method of moments. Noting that the moments of the semicircle law determine it, one computes by combinatorial means the expectation and the variance of the normalized trace of powers of the matrix to find the asymptotics of the moments of the ESDs. The analogue of this for non-normal matrices is to compute the mixed moments, i.e. compute

$$
\int_{\mathbb{C}} w^{k} \bar{w}^{\ell} d L_{B_{n}}(w)=\frac{1}{n} \sum_{i=1}^{n} \lambda_{i}\left(B_{n}\right)^{k} \bar{\lambda}_{i}\left(B_{n}\right)^{\ell} .
$$

For $B_{n}$ non-normal, the RHS of (1.1) cannot be expressed as powers of traces of $B_{n}$ and $B_{n}^{*}$. So the method of moment approach does not work. Another technique that works well for Hermitian matrices is the method of evaluating limiting Stieltjes transform (see [5]). Since the Stieltjes transform of a probability measure is well defined outside its support, and the ESDs of non-normal matrices are supported on $\mathbb{C}$, their Stieltjes transforms fail to capture the spectral measure.

In the 1950's, based on numerical evidences, it was conjectured that the ESD of $B_{n} / \sqrt{n}$, where $B_{n}$ is an $n \times n$ matrix with i.i.d. entries of zero mean and unit variance, converges to the circular law, the uniform distribution on the unit disk in the complex plane. In random matrix literature this conjecture is commonly known as the circular law conjecture.

Using the formula for the joint density function of the eigenvalues Ginibre [26] solved the case when the entries have a complex Gaussian distribution. The case of real Gaussian entries, where a similar formula is available, was settled by Edelman [22]. For the general case when there is no such formula, the problem remained open for a very long time. An approach to the problem, which eventually played an important role in the resolution of the conjecture, was suggested by Girko in the 1980's [27]. However mathematically it contained significant gaps. The first non-Gaussian case (assuming the existence of density for the entries) was rigorously treated by Bai [4], and the first result without the density assumption was obtained by Götze and Tikhomirov [28]. After a series of partial results (see [14] and the references therein), the circular law conjecture 
was established in its full generality in the seminal work of Tao and $\mathrm{Vu}$ [43]:

Theorem 1.1 (Circular law for i.i.d. entries [43, Theorem 1.10]). Let $M_{n}$ be an $n \times n$ random matrix whose entries are i.i.d. complex random variables with zero mean and unit variance. Then the ESD of $\frac{1}{\sqrt{n}} M_{n}$ converges weakly to the uniform distribution on the unit disk on the complex plane, both in probability and in the almost sure sense.

A remarkable feature of Theorem 1.1 is its universality. The asymptotic behavior of the ESD of $M_{n} / \sqrt{n}$ is insensitive to the specific details of the entry distributions as long as they are i.i.d. and have zero mean and unit variance. Since the work of Tao and $\mathrm{Vu}$, there have been numerous attempts to extend the universality of Theorem 1.1 for a wider class of random matrix ensembles. A natural extension would be to prove Theorem 1.1 for matrix ensembles with dependent entries. This has been shown in $[1,2,12,32,33]$.

Another direction to pursue is to study the asymptotics of the ESD of sparse matrices. Sparse matrices are abundant in statistics, neural network, financial modeling, electrical engineering, wireless communications, neuroscience, and in many other fields. We refer the reader to [6, Chapter 7] for other examples, and their relevant references. One model for sparse random matrices is the adjacency matrices of random $d$-regular directed graphs with $d=o(n)$ (for $\left\{a_{n}\right\}$ and $\left\{b_{n}\right\}$, sequences of positive reals, the notation $a_{n}=o\left(b_{n}\right)$ means $\left.\lim _{n \rightarrow \infty} a_{n} / b_{n}=0\right)$. Recently in $[7,20,31]$ the circular law conjecture was established for two different models of random $d$-regular directed graphs.

One of the most natural models for sparse random matrices is the Hadamard product of a matrix of i.i.d. entries with zero mean and unit variance, and a matrix of i.i.d. $\operatorname{Ber}\left(p_{n}\right)$ entries, with $p_{n}=o(1)$. In this paper we focus on the limiting spectral distribution of this class of sparse matrices. When $p_{n}=$ $n^{\alpha-1}$ for some $\alpha \in(0,1)$, it has been shown that, under the assumption of the existence of $(2+\delta)$-th moment of the entries, the ESD of these sparse matrices (properly scaled) converges weakly to the circular law, in probability and almost surely (see [28, 42]). Later in [47] the assumption on the existence of $(2+\delta)$-th moment was removed but the convergence was shown to hold in probability.

In this paper, we prove that the circular law limit continues hold when $n p_{n}$ grows at a sub-polynomial rate (in $n$ ). Namely, we show that under certain moment assumptions of the entries the circular law limit holds for sparse nonHermitian matrices whenever $n p_{n}$ grows at a poly-logarithmic rate. Under an additional assumption on $p_{n}$ (see (1.2) below), the convergence is shown to hold almost surely.

Before stating our result, let us recall the well-known definition of subGaussian random variables.

Definition 1.2. For a random variable $\xi$, the sub-Gaussian norm of $\xi$, denoted by $\|\xi\|_{\psi_{2}}$, is defined as

$$
\|\xi\|_{\psi_{2}}:=\sup _{k \geq 1} k^{-1 / 2}\|\xi\|_{k}, \quad \text { where }\|\xi\|_{k}:=\left(\mathbb{E}|\xi|^{k}\right)^{1 / k} .
$$

If the sub-Gaussian norm is finite, the random variable $\xi$ is called sub-Gaussian. 
We use the following standard notation: for two sequences positive reals $\left\{a_{n}\right\}$ and $\left\{b_{n}\right\}$ we write $a_{n}=\omega\left(b_{n}\right)$ if $b_{n}=o\left(a_{n}\right)$ and $a_{n}=O\left(b_{n}\right)$ if $\limsup _{n \rightarrow \infty} a_{n} / b_{n}<$ $\infty$.

The following is the main result of this article.

Theorem 1.3. Let $A_{n}$ be an $n \times n$ matrix with i.i.d. entries $a_{i, j}=\delta_{i, j} \xi_{i, j}$, where $\delta_{i, j}, i, j \in[n]$ are independent Bernoulli random variables taking value 1 with probability $p_{n} \in(0,1]$, and $\xi_{i, j}, i, j \in[n]$ are real-valued $i . i . d$. centered sub-Gaussian with unit variance.

(i) If $p_{n}$ is such that $n p_{n}=\omega\left(\log ^{2} n\right)$ the ESD of $A_{n} / \sqrt{n p_{n}}$ converges weakly to the circular law, as $n \rightarrow \infty$, in probability.

(ii) There exists a constant $c_{1.3}$, depending only on the sub-Gaussian norm of $\left\{\xi_{i, j}\right\}$, such that if $p_{n}$ satisfies the inequality

$$
n p_{n}>\exp \left(c_{1.3} \sqrt{\log n}\right)
$$

then the conclusion of part (i) holds almost surely.

It will be seen in Section 2 that a key to the proof of Theorem 1.3 is a uniform bound on $s_{\min }\left(A_{n}-w \sqrt{n p_{n}} I_{n}\right)$ for Lebesgue a.e. $w \in \mathbb{C}$, where $s_{\min }(\cdot)$ denotes the smallest singular value. We initiated this work in [9] and showed that the desired bound holds when $w \in \mathbb{R}$. The result of [9] relied on identifying the obstacles of arithmetic nature by methods of Fourier analysis, and using geometry to show that with high probability none of these obstacles realizes. However, even if the matrix $A_{n}$ is real valued, the extension to $w \in \mathbb{C} \backslash \mathbb{R}$ makes the set of potential arithmetic obstacles so rich that it cannot be handled within the framework of the previous argument. This required developing new methods providing both a more precise identification of the arithmetic obstacles arising from the complex structure and entropy bounds showing that with high probability these obstacles are avoided.

The main part of this paper is devoted to find the desired bound on $s_{\min }$ with a probability bound strong enough to apply Borel-Cantelli lemma in order to deduce the almost sure convergence of Theorem 1.3(ii). To remove the condition (1.2) one needs an improvement of [9, Proposition 3.1]. See Remark 7.4 for more details.

It is easy to see that if $p_{n}=\frac{\log n}{n}$, then the number of zero columns of $A_{n}$ is positive (and hence $s_{\min }\left(A_{n}\right)=0$ ) with probability bounded away from zero. So $\frac{\log n}{n}$ is a natural barrier in this set-up. To extend the bound on $s_{\min }$ beyond this barrier one needs to analyze the smallest singular value of the adjacency matrix of "core of the graph", when $A_{n}$ is viewed as the adjacency matrix of directed random weighted graph. We leave this effort to future ventures.

Another key ingredient for the proof of Theorem 1.3 is the bound on the smallish singular values of $\left(A_{n}-w \sqrt{n p_{n}} I_{n}\right)$ (see Theorem 2.10). This is derived by relating the inverse second moment of the singular values to that of the distance of a random vector from a random subspace. This idea originated in [43] and was crucial in relaxing the assumption on the existence of the $(2+\delta)$-th 
moment and prove Theorem 1.1 only under the second moment assumption. To carry out a similar scheme in the sparse set-up one needs to consider random subspaces of dimension $n-m$ with $m=o(n / \log n)$. Concentration inequalities yield a probability bound $\exp \left(-c m p_{n}\right)$, for some $c>0$. To accommodate a union bound we then need $n p_{n}=\omega\left(\log ^{2} n\right)$ which translates to the required lower bound on $p_{n}$ in Theorem 1.3(i).

Remark 1.4 (Sub-Gaussianity assumption). The sub-Gaussianity assumption of Theorem 1.3 is used in Theorem 2.2 to show that $\left\|A_{n}\right\|=O\left(\sqrt{n p_{n}}\right)$, where $\|\cdot\|$ denotes the operator norm. From [9, Remark 1.9] we note that if $\left\{\xi_{i, j}\right\}$ are such that

$$
\mathbb{E}\left|\xi_{i, j}\right|^{h} \leq C^{h} h^{\beta h}, \quad \text { for all } h \geq 1, \text { and for some constants } C \text { and } \beta,
$$

then $\left\|A_{n}\right\|=O\left(\sqrt{n p_{n}}\right)$, for all $p_{n}$ satisfying $n p_{n}=\Omega\left((\log n)^{2 \beta}\right.$ ) (for two sequences of positive reals $\left\{a_{n}\right\}$ and $\left\{b_{n}\right\}$ we write $a_{n}=\Omega\left(b_{n}\right)$ if $b_{n}=O\left(a_{n}\right)$ ). The case $\beta=1 / 2$ corresponds to the sub-Gaussian random variables. So we conclude that if $\left\{\xi_{i, j}\right\}$ satisfies the moment assumption (1.3), for some $\beta \geq$ $1 / 2$, then the conclusion of Theorem 1.3(i) holds for all $p_{n}$ satisfying $n p_{n} \geq$ $\omega\left(\log ^{2} n\right), \Omega\left((\log n)^{2 \beta}\right)$. It is easy to check that $p_{n}$ satisfies $(1.2)$ whenever $n p_{n}=$ $\omega\left((\log n)^{2 \beta}\right)$. Hence, the conclusion of Theorem 1.3(ii) also holds under the moment assumption (1.3). To retain the clarity of presentation we prove Theorem 1.3 for sub-Gaussian random variables.

Remark 1.5 (Circular law limit for shifted sparse matrices). It is well known that the spectrum of normal matrices is stable under small perturbations (see $[3$, Lemma 2.1.19] and [5, Lemma 2.2]). However, for a general non-normal matrix its spectrum is highly sensitive to small perturbations, for example see [41, Section 2.8.1]. So there are no analogues of [3, Lemma 2.1.19] and [5, Lemma 2.2] for an arbitrary non-normal matrix. Nevertheless, in [47] it was shown that if $D_{n}$ is any $n \times n$ matrix with $\operatorname{rank}\left(D_{n}\right)=o(n)$ and $\operatorname{Tr}\left(D_{n} D_{n}^{*}\right)=O\left(n^{2} p_{n}\right)$ then the ESD of $\left(A_{n}+D_{n}\right) / \sqrt{n p_{n}}$ admit a circular law limit. Investigating our proof one can deduce that the ESD of $\left(A_{n}+D_{n}\right) / \sqrt{n p_{n}}$ have a circular law limit for any sequence real diagonal matrices $\left\{D_{n}\right\}$ such that $\left\|D_{n}\right\|=O\left(\sqrt{n p_{n}}\right)$ and $\operatorname{Tr}\left(D_{n}^{2}\right)=o\left(n^{2} p_{n}\right)$. It is possible to modify the proof of Theorem 1.3 to establish the circular law limit for general shifts. We do not pursue this direction here.

We next show that the circular law limit holds for the adjacency matrix of a directed Erdős-Rényi random graph which may be of interest in computer science and graph theory. Let us begin with the relevant definitions.

Definition 1.6. Let $\mathrm{G}_{n}$ be a random directed graph on $n$ vertices, with vertex set $[n]$, such that for every $i \neq j$, a directed edge from $i$ to $j$ is present with probability $p$, independently of everything else. Assume that the graph $\mathrm{G}_{n}$ is simple, i.e. no self-loops or multiple edges are present. We call this graph $\mathrm{G}_{n}$ a directed Erdős-Rényi graph with edge connectivity probability $p$. For any such graph $G_{n}$ we denote $\operatorname{Adj}_{n}:=\operatorname{Adj}\left(G_{n}\right)$ to be its adjacency matrix. That is, for 
any $i, j \in[n]$,

$$
\operatorname{Adj}_{n}(i, j)= \begin{cases}1 & \text { if a directed edge from } i \text { to } j \text { is present in } \mathrm{G}_{n} \\ 0 & \text { otherwise. }\end{cases}
$$

Theorem 1.7. Let $\mathrm{Adj}_{n}$ be the adjacency matrix of a directed Erdös-Rényi graph, with edge connectivity probability $p_{n} \in(0,1)$. Denote $\bar{p}_{n}:=\min \left\{p_{n}, 1-\right.$ $\left.p_{n}\right\}$.

(i) If $p_{n}$ is such that $n \bar{p}_{n}=\omega\left(\log ^{2} n\right)$ the $\mathrm{ESD}$ of $\operatorname{Adj}_{n} / \sqrt{n p_{n}\left(1-p_{n}\right)}$ converges weakly to the circular law, as $n \rightarrow \infty$, in probability.

(ii) There exists an absolute constant $c_{1.7}$ such that if $p_{n}$ satisfies the inequality

$$
n \bar{p}_{n}>\exp \left(c_{1.7} \sqrt{\log n}\right)
$$

then the conclusion of part (i) holds almost surely.

The proof of Theorem 1.7 follows from a relatively standard modification of that of Theorem 1.3. We refer the reader to the arXiv version of this paper [8].

Remark 1.8. Theorem 1.3 and Theorem 1.7 find the asymptotics of the eigenvalues of a large class of sparse non-Hermitian random matrices at a macroscopic level. An interesting question would be to prove the universality of the eigenvalue distribution at the microscopic level. This has been shown for a wide class of Hermitian random matrices (see [11] and references therein). For dense non-Hermitian random matrices, it was shown in [15] that the local circular law holds. In a forthcoming article [10] we establish the same for sparse nonHermitian random matrices.

\section{Outline of the paper}

Section 2 provides a brief outline of the proof techniques of Theorem 1.3. We begin Section 2 with a replacement principle (see Lemma 2.1) which is a consequence of Girko's method. The replacement principle allows us to focus only on the integrability of $\log (\cdot)$ with respect to the ESD of $\widetilde{\boldsymbol{B}}_{n}^{w}:=\left[\left(B_{n}-w I_{n}\right)^{*}\left(B_{n}-\right.\right.$ $\left.\left.w I_{n}\right)\right]^{1 / 2}$ for $w \in \mathbb{C}$, where $B_{n}$ is any random matrix. To implement this scheme one requires a good control on $s_{\min }\left(\widetilde{\boldsymbol{B}}_{n}^{w}\right)$ as well as on its smallish singular values. One also needs to establish weak convergence of the ESDs of $\widetilde{\boldsymbol{B}}_{n}^{w}$.

The required control on $s_{\min }$ and smallish singular values are derived in Theorem 2.2 and Theorem 2.10, and we outline of their proofs in Section 2.1 and Section 2.2, respectively. The limit of the ESDs of $\widetilde{\boldsymbol{B}}_{n}^{w}$ is derived in Theorem 2.11 with the outline of the proof appearing in Section 2.3.

Section 3 - Section 7 are devoted to the proof of Theorem 2.2. Since $s_{\min }\left(M_{n}\right)$ equals the infimum of $\left\|M_{n} u\right\|_{2}\left(\|\cdot\|_{2}\right.$ denotes the Euclidean norm) over all vectors $u$ of unit $\ell_{2}$ norm, we split the unit sphere into three parts: compressible vectors, dominated vectors and the complement of their union. The compressible vectors and dominated vectors are treated with results from [9]. The majority 
of the work is to control infimum over the vectors that are neither compressible nor dominated. Using a result of [37] (see Lemma 3.5 there) this boils down to controlling the inner product of the first column of $\left(A_{n}-w \sqrt{n p_{n}} I_{n}\right)$ and the vector normal to $H_{n}^{w}$, the subspace spanned by the last $(n-1)$ columns of $\left(A_{n}-w \sqrt{n p_{n}} I_{n}\right)$. In Section 7 , it is shown that the last assertion can be proved using Berry-Esséen Theorem. However, the probability bounds obtained from Berry-Esséen Theorem is too weak to prove the almost sure convergence of Theorem 1.3(ii).

In Section 3 - Section 6, we derive a better probability bound that is suitable for the proof of Theorem 1.3(ii). We split the set of vectors into two categories: genuinely complex and essentially real. Roughly speaking, the set of essentially real vectors are those for which the real and the imaginary parts are almost linearly dependent, and its complement is the set of genuinely complex vectors.

In Section 3, we show that the vector normal to $H_{n}^{w}$ has a non-dominated real part, with high probability. We construct a net of small cardinality for the genuinely complex vectors in Section 4. We then use this net in Section 5 and results of Section 3 to show that with high probability, the normal vector cannot be a genuinely complex vector with a sub-exponential (in $\sqrt{n p_{n}}$ ) LCD. A similar result for essentially real vectors is obtained in Section 6 . Then we finish the proof of Theorem 2.2 in Section 7.

In Section 8 we prove Theorem 2.10. The key idea is to show that the distance of any row of $A_{n}$ from any given subspace of relatively small dimension cannot be too small with large probability. This observation together with [43, Lemma A.4] finishes the proof.

Section 9 is devoted to the proof of Theorem 2.11, which establishes the weak convergence of the empirical measure of the singular values of $\left(A_{n} / \sqrt{n p_{n}}-w I_{n}\right)$. The weak convergence is established by appealing to the Lindeberg replacement lemma, which was introduced by Chatterjee in [17], in conjunction with the standard concentration inequalities for the spectral measure of a Hermitian matrix and a truncation argument.

Finally in Section 10, combining the results of Section 7 - Section 9, we finish the proof of Theorem 1.3.

\section{Preliminaries and Proof Outline}

In this section we provide an outline of the proof of Theorem 1.3 and introduce necessary definitions and notation. As mentioned in Section 1, the standard technique to find the limiting spectral distribution of a non-normal matrix is the Girko's method. We refer the reader to [7] for a detailed description of it. The utility of Girko's method, in the context of our set-up, can be captured by the following replacement principle. The replacement principle, which has its origin in [43], gives a sufficient criterion for the ESD of two random matrix ensembles to have the same limit. To state the relevant result, first we introduce a few definitions. A sequence of random variables $\left\{X_{n}\right\}$ is said to be bounded 
in probability if

$$
\lim _{K \rightarrow \infty} \limsup _{n \rightarrow \infty} \mathbb{P}\left(\left|X_{n}\right| \leq K\right)=1
$$

and $\left\{X_{n}\right\}$ is said to be almost surely bounded if

$$
\mathbb{P}\left(\limsup _{n}\left|X_{n}\right|<\infty\right)=1 \text {. }
$$

Next, for a matrix $B_{n}$, we denote $\left\|B_{n}\right\|_{2}$ its Frobenius norm, i.e. $\left\|B_{n}\right\|_{2}:=$ $\sqrt{\operatorname{Tr}\left(B_{n}^{*} B_{n}\right)}$.

Lemma 2.1 (Replacement lemma). (a) Let $B_{n}^{(1)}$ and $B_{n}^{(2)}$ be two sequences of $n \times n$ random matrices, such that

(i) The expression

$$
\frac{1}{n}\left\|B_{n}^{(1)}\right\|_{2}^{2}+\frac{1}{n}\left\|B_{n}^{(2)}\right\|_{2}^{2} \quad \text { is bounded in probability, }
$$

and

(ii) For Lebesgue almost all $w \in \mathbb{D} \subset B_{\mathbb{C}}(0, R) \subset \mathbb{C}$, for some domain $\mathbb{D}$ and some finite $R$, $\frac{1}{n} \log \left|\operatorname{det}\left(B_{n}^{(1)}-w I_{n}\right)\right|-\frac{1}{n} \log \left|\operatorname{det}\left(B_{n}^{(2)}-w I_{n}\right)\right| \rightarrow 0, \quad$ in probability.

Then for every $f \in C_{c}^{2}(\mathbb{C})$ supported on $\mathbb{D}$,

$$
\int f(z) d L_{B_{n}^{(1)}}(w)-\int f(z) d L_{B_{n}^{(2)}}(w) \rightarrow 0, \quad \text { in probability. }
$$

(b) If (2.1) is almost surely bounded and (2.2) holds almost surely then (2.3) holds almost surely as well.

The replacement principle of [43] requires a uniform control on $s_{\min }\left(A_{n}-\right.$ $w \sqrt{n p} I_{n}$ ) for Lebesgue almost every $w \in \mathbb{C}$. Theorem 2.2 (see below) provides such a control only when $w$ is away from the real line. Therefore we need to use Lemma 2.1, borrowed from [7], instead of [43, Theorem 2.1].

Lemma 2.1(a) was proved in [7]. Repeating the proof of [7, Lemma 10.1] one can derive Lemma 2.1(b). Details are left to the reader.

We apply Lemma 2.1 with $B_{n}^{(1)}=\frac{1}{\sqrt{n p_{n}}} A_{n}$ and $B_{n}^{(2)}$ which is the matrix of i.i.d. centered complex Gaussian entries with variance $1 / n$. The assumption (i) is straightforward to verify: it follows from laws of large numbers. It is well known that $\frac{1}{n} \log \left|\operatorname{det}\left(B_{n}^{(2)}-w I_{n}\right)\right|$ admits a limit. Hence, establishing assumption (ii) of Lemma 2.1 boils down to showing that $\log (\cdot)$ is integrable with respect to the empirical measure of the singular values of $B_{n}^{(1)}-w I_{n}$. As $\log (\cdot)$ is unbounded near zero, one needs to establish the weak convergence of the empirical measure of the singular values, find bounds on $s_{\min }$, and show that there are not many singular values in an interval near zero (the unboundedness of $\log (\cdot)$ near infinity is not a problem since the maximal singular value of $B_{n}^{(1)}-w I_{n}$ is $O(1)$ with probability $1-o(1))$. These are the three ingredients of the proof of Theorem 1.3 . 


\subsection{Smallest singular value}

The desired bound on $s_{\min }\left(A_{n}-\sqrt{n p_{n}} w I_{n}\right)$ is derived in the theorem below.

Theorem 2.2. Let $A_{n}$ be an $n \times n$ matrix with i.i.d. entries $a_{i, j}=\delta_{i, j} \xi_{i, j}$, where $\left\{\delta_{i, j}\right\}$ are independent Bernoulli random variables taking value 1 with probability $p_{n} \in(0,1]$, and $\left\{\xi_{i, j}\right\}$ are i.i.d. real-valued centered sub-Gaussian with unit variance. Fix $R \geq 1, r \in(0,1]$ and let $D_{n}$ be a diagonal matrix such that $\left\|D_{n}\right\| \leq R \sqrt{n p_{n}}$ and $\operatorname{Im}\left(D_{n}\right)=r^{\prime} \sqrt{n p_{n}} I_{n}$ for some $r^{\prime}$ with $\left|r^{\prime}\right| \in[r, 1]$. Then there exist constants $0<c_{2.2}, \bar{c}_{2.2}, c_{2.2}^{\prime}, C_{2.2}, C_{2.2}^{\prime}, \bar{C}_{2.2}<\infty$, depending only on $R, r$, and the sub-Gaussian norm of $\left\{\xi_{i, j}\right\}$, such that for any $\varepsilon>0$ we have the following:

(i) If

$$
p_{n} \geq \frac{\bar{C}_{2.2} \log n}{n}
$$

then

$$
\mathbb{P}\left(s_{\min }\left(A_{n}+D_{n}\right) \leq c_{2.2} \varepsilon \exp \left(-C_{2.2} \frac{\log \left(1 / p_{n}\right)}{\log \left(n p_{n}\right)}\right) \sqrt{\frac{p_{n}}{n}}\right) \leq \varepsilon+\frac{C_{2.2}^{\prime}}{\sqrt{n p_{n}}} .
$$

(ii) Additionally, if

$$
\log \left(1 / p_{n}\right)<\bar{c}_{2.2}\left(\log n p_{n}\right)^{2}
$$

then

$$
\begin{aligned}
\mathbb{P}\left(s_{\min }\left(A_{n}+D_{n}\right) \leq c_{2.2} \varepsilon \exp \left(-C_{2.2} \frac{\log \left(1 / p_{n}\right)}{\log \left(n p_{n}\right)}\right) \sqrt{\frac{p_{n}}{n}}\right) \\
\leq \varepsilon+\exp \left(-c_{2.2}^{\prime} \sqrt{n p_{n}}\right) .
\end{aligned}
$$

Remark 2.3. It is easy to check that if $n p_{n}>\exp \left(c_{\star} \sqrt{\log n}\right)$ for some constant $c_{\star}$ then $p_{n}$ satisfies (2.4). Therefore, it is enough to prove Theorem 2.2(ii) under the assumption (2.4) in order to apply it to the proof of Theorem 1.3(ii).

Remark 2.4 (Optimality of Theorem 2.2). It is believed that for $A_{n}$ and $D_{n}$ as in Theorem 2.2 one has that $s_{\min }\left(A_{n}+D_{n}\right) \sim p_{n}^{1 / 2} n^{-1 / 2}$. Hence, for $p_{n} \sim n^{\alpha-1}$ for some $\alpha>0$, Theorem 2.2 gives an optimal lower bound on $s_{\min }\left(A_{n}+D_{n}\right)$. However, when $n p_{n}$ grows at a rate sub-polynomial in $n$, we get an additional factor $n^{-o(1)}$. This is due to the fact that one needs $\rho=o(1)$ in [9, Proposition 3.1]. To obtain the optimal lower bound on $s_{\min }\left(A_{n}+D_{n}\right)$ one needs $\rho=\Omega(1)$ there.

We also add that the optimal probability bound for the event on the LHS of (2.5) is $\varepsilon+\exp \left(-c_{2.2}^{\prime} n p_{n}\right)$. The sub-optimality of the probability bound in (2.5) is again due to the fact that we can only allow $\rho=o(1)$ in [9, Proposition 3.1].

Similar to [9], without loss of generality, we can and will assume that $p \leq$ $c R^{-2}$, for some small positive constant $c$. For larger values of $p$ the entries $a_{i, j}$ have variance bounded below by an absolute constant. In such case, we can 
ignore sparsity and regard entries $a_{i, j}$ as i.i.d. centered sub-Gaussian random variables whose variance is bounded below.

To prove Theorem 2.2 we follow the same scheme as in [9] and borrow some of its results. Recalling the definition of the smallest singular value we have

$$
s_{\min }\left(A_{n}+D_{n}\right)=\inf _{z \in S_{\mathbb{C}}^{n-1}}\left\|\left(A_{n}+D_{n}\right) z\right\|_{2},
$$

where $S_{\mathbb{C}}^{n-1}:=\left\{z \in \mathbb{C}^{n}:\|z\|_{2}=1\right\}$. Thus, to bound $s_{\min }$ we need a lower bound on this infimum. To obtain such a bound we decompose the unit sphere into compressible, dominated, and incompressible vectors, and obtain necessary bounds on the infimum on each of these parts separately. The definitions of compressible, dominated, and incompressible vectors are borrowed from [9]. However, we now need to treat complex shifts of the matrix $A_{n}$ which necessitates a straightforward modification of those definitions to accommodate vectors with complex valued entries. We start with the definition of compressible and incompressible vectors.

Definition 2.5. Fix $m<n$. The set of $m$-sparse vectors is given by

$$
\operatorname{Sparse}(m):=\left\{z \in \mathbb{C}^{n}|| \operatorname{supp}(z) \mid \leq m\right\},
$$

where $|S|$ denotes the cardinality of a set $S$ and $\operatorname{supp}(\cdot)$ denotes the support. Furthermore, for any $\delta>0$, the vectors which are $\delta$-close to $m$-sparse vectors in Euclidean norm, are called $(m, \delta)$-compressible vectors. The set of all such vectors, hereafter will be denoted by $\operatorname{Comp}(m, \delta)$. Thus,

$$
\operatorname{Comp}(m, \delta):=\left\{z \in S_{\mathbb{C}}^{n-1} \mid \exists y \in \operatorname{Sparse}(m) \text { such that }\|z-y\|_{2} \leq \delta\right\} .
$$

The vectors in $S_{\mathbb{C}}^{n-1}$ which are not compressible are defined to be incompressible, and the set of all incompressible vectors is denoted as $\operatorname{Incomp}(m, \delta)$.

Next we define the dominated vectors. These are close to sparse vectors but in a different sense.

Definition 2.6. For any $z \in S_{\mathbb{C}}^{n-1}$, let $\pi_{z}:[n] \rightarrow[n]$ be a permutation which arranges the absolute values of the coordinates of $z$ in a non-increasing order. For $1 \leq m \leq m^{\prime} \leq n$, denote by $z_{\left[m: m^{\prime}\right]} \in \mathbb{C}^{n}$ the vector with coordinates

$$
z_{\left[m: m^{\prime}\right]}(j):=z(j) \cdot \mathbf{1}_{\left[m: m^{\prime}\right]}\left(\pi_{z}(j)\right) .
$$

In other words, we include in $z_{\left[m: m^{\prime}\right]}$ the coordinates of $z$ which take places from $m$ to $m^{\prime}$ in the non-increasing rearrangement of its absolute values. For $\alpha<1$ and $m \leq n$ define the set of vectors with dominated tail as follows:

$$
\operatorname{Dom}(m, \alpha):=\left\{z \in S_{\mathbb{C}}^{n-1} \mid\left\|z_{[m+1: n]}\right\|_{2} \leq \alpha \sqrt{m}\left\|z_{[m+1: n]}\right\|_{\infty}\right\} .
$$

Note that by definition, $\operatorname{Sparse}(m) \cap S_{\mathbb{C}}^{n-1} \subset \operatorname{Dom}(m, \alpha)$, since for $m$-sparse vectors, $z_{[m+1: n]}=0$. 
While studying the behavior of $s_{\min }$ of real shifts of $A_{n}$ in [9], we noted that the control of the infimum over compressible and dominated vectors can be extended when they are viewed as subsets of $S_{\mathbb{C}}^{n-1}$ (cf. [9, Remark 3.10]). So we only need to control the infimum over vectors that are neither compressible nor dominated. The infimum over the incompressible vectors is tackled by associating it with the average distance of a column of the matrix $A_{n}$ from the subspace spanned by the rest of the columns. We use the following result:

Lemma 2.7 (Invertibility via distance [37, Lemma 3.5]). Let $\tilde{A}_{n}$ be any $n \times n$ random matrix. For $j \in[n]$, let $\tilde{A}_{n, j} \in \mathbb{C}^{n}$ be the $j$-th column of $\tilde{A}_{n}$, and let $H_{n, j}$ be the subspace of $\mathbb{C}^{n}$ spanned by $\left\{\tilde{A}_{n, i}, i \in[n] \backslash\{j\}\right\}$. Then for any $\varepsilon, \rho>0$, and $M<n$,

$$
\mathbb{P}\left(\inf _{z \in \operatorname{Incomp}(M, \rho)}\left\|\tilde{A}_{n} z\right\|_{2} \leq \varepsilon \rho^{2} \sqrt{\frac{p}{n}}\right) \leq \frac{1}{M} \sum_{j=1}^{n} \mathbb{P}\left(\operatorname{dist}\left(\tilde{A}_{n, j}, H_{n, j}\right) \leq \rho \sqrt{p} \varepsilon\right) .
$$

We should mention here that Lemma 2.7 can be extended to the case when the event on the LHS of (2.6) is intersected with an event $\Omega$, and in that case Lemma 2.7 continues to hold if the RHS of (2.6) is replaced by intersecting each of the event under the summation sign with the same event $\Omega$ (see also [9, Remark 2.5]). We will actually use this generalized version of Lemma 2.7.

In order to apply Lemma 2.7 in our set-up, denote by $B^{D, n-1}$ the $(n-1) \times n$ matrix obtained by collecting the last $(n-1)$ rows of $\left(A_{n}+D_{n}\right)^{\mathrm{T}}$. Hereafter, for brevity, we will often write $B^{D}$ instead of $B^{D, n-1}$. We note that any unit vector $z$ such that $B^{D} z=0$ is a vector normal to the subspace spanned by the last $(n-1)$ columns of $\left(A_{n}+D_{n}\right)$. Thus, applying Lemma 2.7 and the fact that the columns of $\bar{A}_{n}$ are i.i.d., we see that it is enough to find bounds on $\left\langle A_{n, 1}^{D}, z\right\rangle$, such that $B^{D} z=0$, where $A_{n, 1}^{D}$ is the first column of $\left(A_{n}+D_{n}\right)$.

The small ball probability bounds on $\left\langle A_{n, 1}^{D}, z\right\rangle$ depend on the additive structure of the vector $z$. Following [9], we see that with high probability, we can assume that any $z \in \operatorname{Ker}\left(B^{D}\right)$ is neither compressible nor dominated, where $\operatorname{Ker}\left(B^{D}\right):=\left\{u \in \mathbb{C}^{n}: B^{D} u=0\right\}$. Therefore, it is enough to obtain estimates on the small ball probability for incompressible and non-dominated vectors. To this end, we define the following notion of Lévy concentration function:

Definition 2.8. Let $Z$ be a random variable in $\mathbb{C}^{n}$. For every $\varepsilon>0$, the Lévy concentration function of $Z$ is defined as

$$
\mathcal{L}(Z, \varepsilon):=\sup _{u \in \mathbb{C}^{n}} \mathbb{P}\left(\|Z-u\|_{2} \leq \varepsilon\right)
$$

The Berry-Esséen bound of [35, Theorem 2.2.17] yields a weak control on Lévy concentration function which is enough to prove Theorem 2.2(i). To prove Theorem 2.2(ii) a significant amount of additional work is needed which is the key contribution of this paper. 
To obtain a strong probability bound on the Lévy concentration function, the standard approach is to first quantify the additive structure present in an incompressible vector via the definition of least common denominator (LCD). When the LCD is large, one can derive a good bound on the Lévy concentration function using Esséen's inequality [24] (see also [44, Theorem 6.3]). However, Esséen's inequality does not yield a strong small ball probability estimate for vectors with small values of LCD. Nevertheless, these vectors are shown to admit a special net of small cardinality and therefore one can still apply the union bound to complete the proof. For example, see [9, 37, 38]. One would hope to carry out the same program here. However, when we view the incompressible and non-dominated vectors of small LCD as a subset of $S_{\mathbb{C}}^{n-1}$, its real dimension is twice as large as in the proof [9, Proposition 4.1]. On the other hand, for the real-valued random variables in $A_{n}$, one does not expect to obtain better control on the Lévy concentration function. Thus the proof of [9, Proposition 4.1] breaks down as the bounds on the Lévy concentration function and the size of the net do not match (see also [9, Remark 4.5]).

To tackle this obstacle we decompose the vectors according to the angle between their real and imaginary parts. More precisely, we define the realimaginary de-correlation as follows:

Definition 2.9. Let $z \in \mathbb{C}^{m}$ for some positive integer $m$. Denote $V:=V(z):=$ $\left(\begin{array}{l}x^{\top} \\ y^{\top}\end{array}\right)$, where $z=x+\mathrm{i} y$. Then we denote the real-imaginary de-correlation of $z$ by

$$
d(z):=\left(\operatorname{det}\left(V V^{\top}\right)\right)^{1 / 2} .
$$

This notion of real-imaginary de-correlation was introduced in [39] to study the no-gap deocalization property of the eigenvectors of a wide class of random matrices. In [39] it is termed as "real-complex correlation". Here we deviate from that terminology upon noting that small values of $d(z)$ indicate that the real and the imaginary part of $z$ are close to being linearly dependent.

If a vector $z \in S_{\mathbb{C}}^{n-1}$ has a large value of $d(z)$, then we call this vector genuinely complex, whereas vectors with small real-imaginary de-correlations are termed essentially real vectors (See (5.1) and (6.1) for a precise formulation). The real and imaginary parts of essentially real vectors being almost linearly dependent it allows us to construct a net whose cardinality is a polynomial of degree $n$ in terms of the mesh. Therefore, one can use the small ball probability estimates from [9] to show that with high probability, there does not exist any essentially real vector in the kernel of $B^{D}$ with a small LCD.

The analysis of genuinely complex vectors is more delicate. Following the recent work of [39] we define a notion of a two-dimensional LCD. Roughly speaking the two-dimensional LCD $D_{2}(\cdot)$ of $z=x+\mathrm{i} y$ identifies a non-trivial $\theta^{\star}(z):=\left(\theta_{1}^{\star}(z), \theta_{2}^{\star}(z)\right) \in \mathbb{R}^{2}$ such that $\theta_{1}^{\star}(z) x+\theta_{2}^{\star}(z) y$ is close to an integer point and $\theta^{\star}(z)$ has the least possible Euclidean norm among all such choices. See Definition 4.3 for a precise formulation. Using a result of [39] (Theorem 7.5 there) we show that the small ball probability bound of genuinely complex vectors decays roughly as the inverse of the $(2 n)$-th power of $D_{2}(\cdot)$ (see the bound in (5.7)). This 
probability bound balances the cardinality of the net of genuinely complex vectors for which $\Delta\left(z_{\text {small }}\right):=\left\|\theta_{1}^{\star}\left(z_{\text {small }}\right) x_{\text {small }}+\theta_{2}^{\star}\left(z_{\text {small }}\right) y_{\text {small }}\right\|_{2}$ (precise definition of $\Delta(\cdot)$ can be found in Definition 4.4) is large, where $z_{\text {small }}=x_{\text {small }}+\mathrm{i} y_{\text {small }}$ is the part of $z$ containing the coordinates of small modulus. It allows us to take the union bound over the net of such vectors. To treat the remaining set of genuinely complex vectors, using results from [9], we show that, with high probability, there cannot exist a vector $z \in \operatorname{Ker}\left(B^{D}\right)$ with a dominated real part. This additional observation then shows that for any $z \in \operatorname{Ker}\left(B^{D}\right)$ the quantity $\Delta\left(z_{\text {small }}\right)$ must also be large. This finishes the outline of the proof of Theorem 2.2 .

\subsection{Intermediate singular values}

We also need to show that there are not too many singular values of $\left(A_{n}-\right.$ $\left.w \sqrt{n p} I_{n}\right)$ in a small interval around zero. The following theorem does that job. Before stating the theorem, for $i \in[n]$, let us denote $s_{i}(\cdot)$ to be the $i$-th largest singular value.

Theorem 2.10. Let $A_{n}$ be an $n \times n$ matrix whose entries are $\left\{\xi_{i, j} \delta_{i, j}\right\}_{i, j=1}^{n}$ where $\left\{\xi_{i, j}\right\}_{i, j=1}^{n}$ are i.i.d. real-valued random variables with zero mean and unit variance, and $\left\{\delta_{i, j}\right\}_{i, j=1}^{n}$ are i.i.d. $\operatorname{Ber}\left(p_{n}\right)$ random variables. There exist con-

stants $c_{2.10}$ and $C_{2.10}{ }^{1}$ such that the following holds: Let $\psi: \mathbb{N} \mapsto \mathbb{N}$ be such that $\psi(n)<n$ and $\min \left\{p_{n} \psi(n), \psi^{2}(n) / n\right\} \geq C_{2.10} \log n$. Then for any $w \in B_{\mathbb{C}}(0,1)$ we have

$$
\mathbb{P}\left(\bigcup_{i=3 \psi(n)}^{n-1}\left\{s_{n-i}\left(\frac{A_{n}}{\sqrt{n p_{n}}}-w I_{n}\right) \leq c_{2.10} \frac{i}{n}\right\}\right) \leq \frac{2}{n^{2}}
$$

To prove Theorem 2.10 we follow the approach of [43], which was adapted to the sparse case in $[13,47]$. We first show that the distance of any row of $A_{n}$ from any given subspace of not very large dimension cannot be too small with large probability. This observation together with a result from [43] finishes the proof.

\subsection{Weak convergence}

Recall that to show the integrability of $\log (\cdot)$ we further need to establish the weak convergence of the empirical measure of the singular values of $\frac{1}{\sqrt{n p}} A_{n}-$ $w I_{n}$. Define

$$
\boldsymbol{A}_{n}^{w}:=\left[\begin{array}{cc}
0 & \frac{1}{\sqrt{n p}} A_{n}-w I_{n} \\
\frac{1}{\sqrt{n p}} A_{n}^{*}-\bar{w} I_{n} & 0
\end{array}\right]
$$

\footnotetext{
${ }^{1}$ the constants $c_{2.10}$ and $C_{2.10}$ can potentially depend on the tail of the distribution of $\left\{\xi_{i}\right\}_{i=1}^{n}$.
} 
and denote by $\nu_{n}^{w}$ the ESD of $\boldsymbol{A}_{n}^{w}$. It can be easily checked that $\nu_{n}^{w}$ is the symmetrized version of the empirical measure of the singular values $\frac{1}{\sqrt{n p}} A_{n}-$ $w I_{n}$. Thus, it is enough to prove the weak convergence of $\nu_{n}^{w}$.

Theorem 2.11. (i) Let $A_{n}$ be an $n \times n$ matrix with entries $a_{i, j}=\delta_{i, j} \cdot \xi_{i, j}$, where $\delta_{i, j}$ are i.i.d. Bernoulli random variables with $\mathbb{P}\left(\delta_{i, j}=1\right)=p_{n}$, and $\xi_{i, j}$ are centered i.i.d. real-valued random variables with unit variance. Assume $p_{n}=\omega\left(\frac{\log n}{n}\right)$. Fix any $w \in B_{\mathbb{C}}(0,1)$. Then there exists a probability measure $\nu_{\infty}^{w}$ such that $\nu_{n}^{w}$ converges weakly to $\nu_{\infty}^{w}$, in probability.

(ii) If additionally $\left\{\xi_{i, j}\right\}_{i, j=1}^{n}$ have finite fourth moment and $\sum_{n=1}^{\infty}\left(n^{2} p_{n}\right)^{-1}<$ $\infty$ then the above convergence holds almost surely.

To prove Theorem 2.11 we first apply a standard truncation technique which shows that it is enough to prove the weak convergence of $\nu_{n}^{w}$ to $\nu_{\infty}^{w}$ for bounded $\left\{\xi_{i, j}\right\}_{i, j=1}^{n}$ (see Lemma 9.1). This truncation argument requires the additional assumptions of part (ii) of Theorem 2.11 to establish the almost sure convergence.

It is well known that $\nu_{G_{n}}^{w}$, the symmetrized version of the empirical law of the singular values of $\frac{1}{\sqrt{n}} G_{n}-w I_{n}$, where $G_{n}$ is a complex Ginibre matrix, converges weakly, almost surely to $\nu_{\infty}^{w}$. Therefore, to obtain Theorem 2.11 it is enough to show that for bounded $\left\{\xi_{i, j}\right\}_{i, j=1}^{n}$, the signed measure $\nu_{n}^{w}-\nu_{G_{n}}^{w}$ converges weakly to the point mass to zero, as $n \rightarrow \infty$, in probability or almost surely, depending on the sparsity parameter $p$.

This is done in Section 9 using the following two-fold argument. First we establish that both the random probability measures $\nu_{n}^{w}$ and $\nu_{G_{n}}^{w}$ are close to their expectations, denoted hereafter by $\mathbb{E} \nu_{n}^{w}$ and $\mathbb{E} \nu_{\boldsymbol{G}_{n}}^{w}$, respectively. This step uses standard concentration inequalities for the spectral measure of Hermitian random matrices.

To complete the proof of Theorem 2.11 we then need to show that $\mathbb{E} \nu_{n}^{w}$ and $\mathbb{E} \nu_{\boldsymbol{G}_{n}}^{w}$ are themselves close to each other. Here we appeal to the Lindeberg replacement principle which was introduced to the random matrix theory in [17, 18] to prove the semicircle law for random symmetric matrices with exchangeable entries on and above the diagonal. Subsequently, this technique has been used on numerous occasions in the random matrix theory literature.

\section{The structure of the kernel: Vectors with non-dominated real part}

Recall from Section 2.1 that the main challenge in proving Theorem 2.2 is to show that there does not exist a genuinely complex vector $z \in \operatorname{Ker}\left(B^{D}\right)$ with a small two-dimensional LCD. As a first step we show that for any $z \in \operatorname{Ker}\left(B^{D}\right)$, its real part must have a non-dominated component with high probability. This is shown in the following result, which is the main result of this section. Before stating the result let us introduce some notation, which is borrowed from [39]: For a number $M<n / 2$, to be determined during the course of the proof, we denote by $\operatorname{small}(z)$ the set of the $(n-M)$ coordinates of $z$ having the smallest 
absolute values. The ties are broken arbitrarily. We also write $z_{\text {small }}=x_{\text {small }}+$ $\mathrm{i} y_{\text {small }}:=z_{\text {small }(z)}$. Hereafter, we drop the subscript in $p_{n}$ and for ease we write $p$ instead.

Proposition 3.1. Let $A_{n}$ be a matrix with i.i.d. entries $a_{i, j}=\xi_{i j} \delta_{i j}$, where $\left\{\xi_{i j}\right\}$ are i.i.d. centered real-valued random variables with unit variance and finite fourth moment, and $\left\{\delta_{i j}\right\}$ are i.i.d. $\operatorname{Ber}(p)$ random variables. Set

$$
\ell_{0}:=\left\lceil\frac{\log 1 /(8 p)}{\log \sqrt{p n}}\right\rceil .
$$

Fix $r \in(0,1]$ and $R \geq 1$ such that $\operatorname{Im}\left(D_{n}\right)=r^{\prime} \sqrt{n p} I_{n}$ with $\left|r^{\prime}\right| \in[r, 1]$ and $\left\|D_{n}\right\| \leq R \sqrt{n p}$. Fix another positive real $K \geq 1$. Then there exist constants $C_{3.1}, \widetilde{C}_{3.1}, c_{3.1}$, and $\bar{c}_{3.1}$, depending only on $r, R, K$, and the fourth moment of $\left\{\xi_{i j}\right\}$, such that the following holds. Denote $\rho:=\left(\widetilde{C}_{3.1}(K+R)\right)^{-\ell_{0}-6}$ and assume that

$$
c_{3.1} \rho^{5} p n>1 .
$$

Set $M=C_{3.1} \rho^{-4} p^{-1}$. Then

$$
\begin{aligned}
\mathbb{P}\left(\exists z \in \operatorname{Ker}\left(B^{D}\right) \cap S_{\mathbb{C}}^{n-1}:\left\|\frac{x_{\text {small }}}{\left\|x_{\text {small }}\right\|_{2}}\right\|_{\infty} \geq \rho p^{1 / 2}\right. \text { and } \\
\left.\qquad\left\|A_{n}\right\| \leq K \sqrt{n p}\right) \leq \exp \left(-\bar{c}_{3.1} n p\right) .
\end{aligned}
$$

Remark 3.2. For clarity we only prove Proposition 3.1 for $r^{\prime} \in[r, 1]$. It will be evident that the proof of the case $r^{\prime} \in[-1,-r]$ is exactly the same. We spare the details.

The key to the proof of Proposition 3.1 is in showing that if the real part of a vector $z$ is compressible then $\left\|B^{D} z\right\|_{2}$ cannot be too small. This is derived in the following lemma:

Lemma 3.3. Let $B^{D}, A_{n}, \rho, K, R, r$, and $r^{\prime}$ be as in Proposition 3.1. Then there exist constants $0<c_{3.3}, c_{3.3}^{\prime}, c_{3.3}^{\prime \prime}, \bar{c}_{3.3}<\infty$, depending only on $K, R, r$, and the fourth moment of $\left\{\xi_{i, j}\right\}$, such that for any $p^{-1} \leq M \leq c_{3.3}^{\prime} n / \log (1 / \rho)$,

$$
\begin{aligned}
& \mathbb{P}\left(\exists z=x+\mathrm{i} y \in S_{\mathbb{C}}^{n-1}:\left\|B^{D} z\right\|_{2} \leq c_{3.3} \rho \sqrt{n p},\right. \\
& \left.\left\|x_{\text {small }}\right\|_{2} \leq c_{3.3}^{\prime \prime} \rho, \text { and }\left\|A_{n}\right\| \leq K \sqrt{n p}\right) \leq \exp \left(-\bar{c}_{3.3} n p\right) .
\end{aligned}
$$

To prove Lemma 3.3 we borrow results from [9]. In [9, Proposition 3.1] we showed that, with high probability, there does not exist any real-valued compressible or dominated vector $z$ such that $\left\|\operatorname{Re}\left(B^{D}\right) z\right\|_{2}$ is small, where $\operatorname{Re}\left(B^{D}\right)$ denotes the real part of the matrix $B^{D}$. In [9, Remark 3.10] it was also argued that the same conclusion holds for $\left\|B^{D} z\right\|_{2}$ when $z$ is now allowed to be complex valued. We will need this result to prove Lemma 3.3. For completeness we state it below. 
Proposition 3.4 ([9, Proposition 3.1, Remark 3.10]). Let $A_{n}$ be as in Proposition 3.1. Fix $K, R \geq 1$, and assume that $D_{n}$ is a non-random diagonal matrices with complex entries such that $\left\|D_{n}\right\| \leq R \sqrt{p n}$. Then there exist constants $0<c_{3.4}, \bar{c}_{3.4}, c_{3.4}^{\prime}, C_{3.4}, \widetilde{C}_{3.4}, \bar{C}_{3.4}<\infty$, depending only on $K, R$, and the fourth moment of $\left\{\xi_{i j}\right\}$, such that for

$$
\frac{\bar{C}_{3.4} \log n}{n} \leq p \leq \frac{1}{10}
$$

and any $p^{-1} \leq M \leq c_{3} .4^{n,}$ we have

$$
\begin{aligned}
& \mathbb{P}\left(\exists z \in \operatorname{Dom}\left(M,\left(C_{3.4}(K+R)\right)^{-4}\right) \cup \operatorname{Comp}(M, \rho)\right. \\
& \left.\quad\left\|\left(A_{n}+D_{n}\right) z\right\|_{2} \leq c_{3.4}^{\prime}(K+R) \rho \sqrt{n p} \text { and }\left\|A_{n}\right\| \leq K \sqrt{p n}\right) \leq \exp \left(-\bar{c}_{3.4} p n\right),
\end{aligned}
$$

where $\rho=\left(\widetilde{C}_{3.4}(K+R)\right)^{-\ell_{0}-6}$ and $\ell_{0}$ are as in Proposition 3.1 .

Observe that Proposition 3.4 is stated for the square matrix $A_{n}$. To prove Lemma 3.3 we need a version of Proposition 3.4 for $(n-1) \times n$ matrices. As noted in [9, Remark 3.9] this follows from an easy adaptation. So, without loss of generality we will use Proposition 3.4 also for $(n-1) \times n$ matrices. The final ingredient for the proof of Lemma 3.3 is an estimate on the Lévy concentration function for incompressible and non-dominated vectors. Such an estimate was derived in [9, Corollary 3.7] for real valued vectors and matrices with zero diagonal and i.i.d. off-diagonal entries. One can investigate its proof to convince oneself that the same proof works for complex valued vectors and matrices with i.i.d. entries. We state this modified version below.

Lemma 3.5 ([9, Corollary 3.7]). Let $A_{n}$ be as in Proposition 3.1. Then for any $\alpha>1$, there exist $\beta, \gamma>0$, depending on $\alpha$ and the fourth moment of $\left\{\xi_{i j}\right\}$, such that for $z \in \mathbb{C}^{n}$, satisfying $\|z\|_{\infty} /\|z\|_{2} \leq \alpha \sqrt{p}$, we have

$$
\mathcal{L}\left(A_{n} z, \beta \cdot \sqrt{p n}\|z\|_{2}\right) \leq \exp (-\gamma n) .
$$

We now proceed to the proof of Lemma 3.3.

Proof of Lemma 3.3. The proof is based on ideas from [25]. For ease of writing let us write $c_{0}:=\left(C_{3.4}(K+R)\right)^{-4}$. We also denote

$$
\begin{aligned}
\Omega_{D, C}:=\left\{\operatorname{Re}\left(B^{D}\right):\right. & \exists z \in \operatorname{Dom}\left(M, c_{0}\right) \cup \operatorname{Comp}(M, \rho) \\
& \left.\left\|B^{D} z\right\|_{2} \leq c_{3.4}^{\prime}(K+R) \rho \sqrt{n p} \text { and }\left\|A_{n}\right\| \leq K \sqrt{n p}\right\},
\end{aligned}
$$

Using Proposition 3.4 we see that $\mathbb{P}\left(\Omega_{D, C}\right) \leq \exp \left(-\bar{c}_{3.4} n p\right)$. We now make the following claim.

Claim. Fix any $J \subset[n]$ of cardinality $M$ and let

$$
\mathcal{Z}_{J}^{\prime}:=\left\{z=x+\mathrm{i} y:\left\|x_{\text {small }}\right\|_{2} \leq c^{\prime \prime} \rho \text { and } \operatorname{supp}\left(x_{[1: M]}\right) \subset J\right\},
$$


for some small constant $c^{\prime \prime}$ to be determined later. Then

$$
\mathbb{P}\left(\left\{\exists z \in \mathcal{Z}_{J}^{\prime} \text { such that }\left\|B^{D} z\right\|_{2} \leq c \rho \sqrt{n p}\right\} \cap \Omega_{D, C}^{c}\right) \leq \exp (-\bar{c} n)
$$

for some small constants $c$ and $\bar{c}$.

The conclusion of the lemma immediately follows from the claim by taking a union bound over $J \subset[n]$, such that $|J|=M$. Thus we now only need to prove the claim.

To prove this claim we will first show that if $z \in \mathcal{Z}_{J}^{\prime}$ such that $\left\|B^{D} z\right\|_{2}$ is small, then $y$, the imaginary part of $z$, belongs to a small neighborhood of a linear image of the subspace spanned by the largest $M$ coordinates of $x$, the real part of $z$. This together with the fact that $\left\|x_{\text {small }}\right\|_{2}$ is small enables us to obtain a net of $\mathcal{Z}_{J}^{\prime}$ with small cardinality. Finally using the estimate on Lévy concentration function of Lemma 3.5 and the union bound we finish the proof of the claim. Below we carry out the details.

Fix any $J \subset[n]$ and let $\left.\operatorname{Re}\left(B^{D}\right)\right|_{J}$ denote the sub-matrix induced by the columns of $\operatorname{Re}\left(B^{D}\right)$ indexed by $J$. We first condition on a realization of $\left.\operatorname{Re}\left(B^{D}\right)\right|_{J}$ and show that for every such realization the conditional probability of the event in the claim is less than $e^{-\bar{c} n}$. Then taking an average over the realizations of $\left.\operatorname{Re}\left(B^{D}\right)\right|_{J}$ the proof will be completed.

So let us assume that $z \in \mathcal{Z}_{J}^{\prime}$ be such that $\left\|B^{D} z\right\|_{2} \leq c \rho \sqrt{n p}$. Then we see that

$$
\left\|\operatorname{Re}\left(B^{D}\right) x-\operatorname{Im}\left(B^{D}\right) y\right\|_{2} \leq\left\|B^{D} z\right\|_{2} \leq c \rho \sqrt{n p}
$$

Notice that $\left\|x_{[M+1: n]}\right\|_{2} \leq\left\|x_{\text {small }}\right\|_{2}$ as $x_{[M+1: n]}$ consists of the smallest in the absolute value coordinates of $x$. Since

$$
\left\|\left.\operatorname{Re}\left(B^{D}\right)\right|_{J^{c}}\right\| \leq\left\|\operatorname{Re}\left(B^{D}\right)\right\| \leq\left\|B^{D}\right\| \leq\left\|A_{n}\right\|+\left\|D_{n}\right\| \leq(K+R) \sqrt{n p},
$$

applying the triangle inequality we further deduce that

$$
\begin{aligned}
\left\|\operatorname{Im}\left(B^{D}\right) y-\left.\operatorname{Re}\left(B^{D}\right)\right|_{J} x_{[1: M]}\right\|_{2} & \leq c \rho \sqrt{n p}+\left\|\left.\operatorname{Re}\left(B^{D}\right)\right|_{J^{c}} x_{[M+1: n]}\right\|_{2} \\
& \leq c \rho \sqrt{n p}+\left\|\left.\operatorname{Re}\left(B^{D}\right)\right|_{J^{c}}\right\| \cdot\left\|x_{\text {small }}\right\|_{2} \\
& \leq 2 c \rho \sqrt{n p}
\end{aligned}
$$

where in the last step we choose $c^{\prime \prime}$ so that $c^{\prime \prime}(K+R) \leq c$.

Hereafter, we write $\operatorname{Im}\left(B^{D}\right)$ to denote the imaginary part of the matrix $B^{D}$. Hence $\operatorname{Im}\left(B^{D}\right)$ is a $(n-1) \times n$ matrix whose first column is zero and the last $(n-1)$ columns form a diagonal matrix whose entries are all equal to $r^{\prime} \sqrt{n p}$. Therefore denoting $\left.y\right|_{[2: n]}$ to be the $(n-1)$ dimensional vector consisting of the last $(n-1)$ coordinates of $y$ we further have that

$$
\left\|\left.y\right|_{[2: n]}-\left.\frac{1}{r^{\prime} \sqrt{n p}} \operatorname{Re}\left(B^{D}\right)\right|_{J} x_{[1: M]}\right\|_{2} \leq 2 r^{\prime-1} c \rho \leq 2 r^{-1} c \rho .
$$

Thus (3.4) implies that the vector $\left.y\right|_{[2: n]}$ belongs to a $\left(2 r^{-1} c \rho\right)$-neighborhood of the linear subspace $\mathscr{E}_{J}^{\prime}:=\operatorname{span}\left(\left.\operatorname{Re}\left(B^{D}\right)\right|_{J} \mathbb{R}^{J}\right) \subset \mathbb{R}^{n-1}$. Since $\left\|x_{[M+1: n]}\right\|_{2} \leq$ 
$c^{\prime \prime} \rho \leq r^{-1} c \rho$ we have that for any $z \in \mathcal{Z}_{J}^{\prime}$, such that $\left\|B^{D} z\right\|_{2} \leq c \rho \sqrt{n p}$, belongs to a $\left(3 r^{-1} c \rho\right)$-neighborhood of the set

$$
\mathscr{E}_{J}:=\left\{x+\mathrm{i} y: \operatorname{supp}(x) \subset J,\left.y\right|_{[2: n]} \in \mathscr{E}_{J}^{\prime}, y_{1} \in[-1,1]\right\},
$$

with $\operatorname{dim}\left(\mathscr{E}_{J}\right) \leq 2 M+1$. Since $\mathcal{Z}_{J}^{\prime} \subset S_{\mathbb{C}}^{n-1}$, applying the triangle inequality and choosing $c \leq r / 3$ we further see that every vector in $z \in \mathcal{Z}_{J}^{\prime}$, such that $\left\|B^{D} z\right\|_{2} \leq c \rho \sqrt{n p}$, belongs to a $\left(3 r^{-1} c \rho\right)$-neighborhood of $\left(2 B_{\mathbb{C}}^{n}\right) \cap \mathscr{E}_{J}$. Therefore we can choose a $\left(r^{-1} c \rho\right)$-net $\mathcal{N} \subset\left(2 B_{\mathbb{C}}^{n}\right) \cap \mathscr{E}_{J}$ of cardinality

$$
|\mathcal{N}| \leq\left(\frac{12}{c \rho}\right)^{2 M+1} \leq \exp (3 M \log (12 /(c \rho))) .
$$

Note that, using the triangle inequality we see that $\mathcal{N}$ is $\left(4 r^{-1} c \rho\right)$-net of the set of all vectors $z \in \mathcal{Z}_{J}^{\prime}$ such that $\left\|B^{D} z\right\|_{2} \leq c \rho \sqrt{n p}$. Thus, for a $z \in \mathcal{Z}_{J}^{\prime}$ with $\left\|B^{D} z\right\|_{2} \leq c \rho \sqrt{n p}$, there must exist at least one $w \in \mathcal{N}$ such that $\left\|B^{D} w\right\|_{2} \leq$ $5 r^{-1}(K+R) c \rho \sqrt{n p}$. Now shrink $c$ such that $10 r^{-1} c \leq c_{3.4}^{\prime}$. With this choice of the constant $c$ we see that $w \notin \operatorname{Dom}\left(M, c_{0}\right) \cup \operatorname{Comp}(M, \rho)$ on the event $\Omega_{D, C}^{c}$.

However, for any $w \notin \operatorname{Dom}\left(M, c_{0}\right)$ we have

$$
\frac{\left\|w_{[M+1: n]}\right\|_{\infty}}{\left\|w_{[M+1: n]}\right\|_{2}} \leq\left(c_{0} \sqrt{M}\right)^{-1} \leq c_{0}^{-1} \sqrt{p}
$$

where in the last step we used the fact that $M \geq p^{-1}$. Thus applying Lemma 3.5 there exists constants $c_{\star}$ and $\bar{c}$ such that

$$
\begin{aligned}
& \mathbb{P}\left(\left\|B^{D} w\right\|_{2} \leq c_{\star} \rho \sqrt{n p}\left|\operatorname{Re}\left(B^{D}\right)\right|_{J}\right) \\
\leq & \mathcal{L}\left(\left.\operatorname{Re}\left(B^{D}\right)\right|_{J^{c}} w_{[M+1: n]}, c_{\star}\left\|w_{[M+1: n]}\right\|_{2} \sqrt{n p}\right) \leq \exp (-2 \bar{c} n),
\end{aligned}
$$

Hence, by the union bound,

$$
\mathbb{P}\left(\exists w \in \mathcal{N}:\left\|B^{D} w\right\|_{2} \leq c_{\star} \rho \sqrt{n p}\left|\operatorname{Re}\left(B^{D}\right)\right|_{J}\right) \leq|\mathcal{N}| \cdot \exp (-2 \bar{c} n) \leq \exp (-\bar{c} n),
$$

where the last step follows from the bound (3.5) and the fact that $M \log (1 / \rho)<$ $c^{\prime} n$ for a sufficiently chosen small constant $c^{\prime}$. Thus shrinking $c$ again such that $5 r^{-1}(K+R) c \leq c_{\star}$ we obtain that

$$
\mathbb{P}\left(\left\{\exists z \in \mathcal{Z}_{J}^{\prime} \text { such that }\left\|B^{D} z\right\|_{2} \leq c \rho \sqrt{n p}\right\} \cap \Omega_{D, C}^{c}\left|\operatorname{Re}\left(B^{D}\right)\right|_{J}\right) \leq \exp (-\bar{c} n) .
$$

Finally taking an average over all the realizations of $\left.\operatorname{Re}\left(B^{D}\right)\right|_{J}$ completes the proof.

We are now ready to prove Proposition 3.1. 
Proof of Proposition 3.1. Let $z \in \operatorname{Ker}\left(B^{D}\right) \cap S_{\mathbb{C}}^{n-1}$. Assume that the event $\Omega_{D, C}^{c}$ defined above occurs, so $z \notin \operatorname{Dom}\left(M, c_{0}\right) \cup \operatorname{Comp}(M, \rho)$. We note that if $c_{3.1}$ is chosen sufficiently small then the assumption (3.1) implies that

$$
\frac{M \log (1 / \rho)}{c_{3.3^{n}}^{\prime}}<1,
$$

whenever $n$ is large enough. So Lemma 3.3 can be applied, which implies that, with high probability, $\left\|x_{\text {small }}\right\|_{2}>c_{3.3}^{\prime \prime} \rho$. On the other hand,

$$
\left\|x_{\text {small }}\right\|_{\infty} \leq\left\|z_{\text {small }}\right\|_{\infty} \leq \frac{1}{c_{0} \sqrt{M}}
$$

Combining the last two inequalities, we show that on the event $\Omega_{D, C}^{c}$,

$$
\frac{\left\|x_{\text {small }}\right\|_{\infty}}{\left\|x_{\text {small }}\right\|_{2}} \leq \frac{1}{c_{3.3}^{\prime \prime} \rho c_{0} \sqrt{M}}
$$

and the result follows upon choosing $C_{3.1}$ sufficiently large.

Remark 3.6. Note that the inequality (3.6) continues to hold even if the constant $C_{3.1}$ is increased without changing other constants $\widetilde{C}_{3.1}, c_{3.1}$, and $\bar{c}_{3.1}$, appearing in Proposition 3.1. This implies that, if needed, we can arbitrarily increase the constant $C_{3.1}$. This observation will be used later in the paper.

\section{Net construction: Genuinely complex case}

In this section we show that the set of genuinely complex vectors admits a net of small cardinality. We begin with the relevant definitions.

Definition 4.1. For $y>0$, denote $\log _{1}(y):=\log y \cdot \mathbb{I}(y \geq e)$. Fixing $L \geq 1$, for a non-zero vector $x \in \mathbb{R}^{m}$, we set

$$
D_{1}(x):=\inf \left\{\theta>0: \operatorname{dist}\left(\theta x, \mathbb{Z}^{m}\right)<2^{5} L \sqrt{\log _{1} \frac{\|\theta x\|_{2}}{2^{6} L}}\right\} .
$$

If $V$ is a $2 \times m$ matrix, define

$$
D_{2}(V):=\inf \left\{\|\theta\|_{2}: \theta \in \mathbb{R}^{2}, \operatorname{dist}\left(V^{\top} \theta, \mathbb{Z}^{m}\right)<L \sqrt{\log _{1} \frac{\left\|V^{\top} \theta\right\|_{2}}{2^{8} L}}\right\} .
$$

We will call the first version of the LCD one-dimensional, and the second one two-dimensional. Note that $D_{1}(\cdot)$ matches with the definition of the LCD used in [9] up to constants.

Remark 4.2. The different powers of 2 appearing in the definitions (4.1) and (4.2) play only a technical role. They do not affect most of the proof, and they will be needed in Section 6 to compare the one and the two-dimensional LCD for almost real vectors (see Lemma 6.1). 
Observe that $D_{1}(\cdot)$ and $D_{2}(\cdot)$ are defined for real-valued vectors and matrices, respectively. However, both these notions can be extended for complex valued vectors by the following simple adaptation.

Definition 4.3. Consider a complex vector $z=x+\mathrm{i} y \in \mathbb{C}^{m}$. Denote $\tilde{z}:=$ $\tilde{z}(z):=\left(\begin{array}{l}x \\ y\end{array}\right) \in \mathbb{R}^{2 m}$, and define a $2 \times m$ matrix $V:=V(z):=\left(\begin{array}{l}x^{\top} \\ y^{\top}\end{array}\right)$. Using these two different representations of $z \in \mathbb{C}^{m}$, we now define:

$$
D_{2}(z):=D_{2}(V) \text { and } \quad D_{1}(z):=D_{1}(\tilde{z}) .
$$

Let us assume that the infimum in (4.2) is attained at $\theta^{\star}$. Then from Definition 4.1 we have that $D_{2}(V)$ equals $\left\|\theta^{\star}\right\|_{2}$. We will see below that the cardinality of the desired net for the genuinely complex vectors also depends on $\left\|V^{\top} \theta^{\star}\right\|_{2}$. However, the infimum in (4.2) is not always achieved. Hence, we have the following definition.

Definition 4.4. For a real-valued $2 \times m$ matrix $V$, define

$$
\begin{aligned}
\Delta(V):=\liminf _{\tau \rightarrow 1+}\left\{\left\|V^{\top} \theta\right\|_{2}: \operatorname{dist}\left(V^{\top} \theta, \mathbb{Z}^{m}\right)<L \sqrt{\log _{1} \frac{\left\|V^{\top} \theta\right\|_{2}}{2^{8} L}},\right. & \\
\|\theta\|_{2} & \left.\leq \tau D_{2}(V)\right\} .
\end{aligned}
$$

As before, for a $z \in \mathbb{C}^{m}$, we define $\Delta(z):=\Delta(V)$ where $V=V(z)$. For later use let us note that for any $z \in S_{\mathbb{C}}^{m-1}$

$$
d(z) D_{2}(z) \leq \Delta(z) \leq D_{2}(z)
$$

where $d(z)$ denotes the real-imaginary de-correlation of $z$ appearing in Definition 2.9. Indeed, the inequalities (4.3) are immediate from the fact that the singular values of $V^{\top}$ are bounded by one and $d(z)$ is the product of the singular values of $V^{\top}$.

Remark 4.5. We take $L=\left(\delta_{0} p\right)^{-1 / 2}$, where $\delta_{0} \in(0,1)$ is a universal constant as in [9, Remark 2.7].

Equipped with the above definitions, we consider the following simple reduction. Fix $M<n / 2, z \in S_{\mathbb{C}}^{n-1}$, and let $J=\operatorname{small}(z)$. It can be easily verified that for any $z \in \mathbb{C}^{n}$ there exists a $\tau \in[0,2 \pi)$ such that $z_{J}=e^{\mathrm{i} \tau}\left(w_{1}+\mathrm{i} w_{2}\right)$, where $w_{1} \perp w_{2}$ and $\left\|w_{2}\right\|_{2} \leq\left\|w_{1}\right\|_{2}$. As $z_{J} \in \operatorname{Ker}(B)$ if and only if $e^{-\mathrm{i} \tau} z_{J} \in \operatorname{Ker}(B)$, without loss of generality, we can only consider the following set

$$
\begin{aligned}
\mathcal{Z}:=\left\{z \in S_{\mathbb{C}}^{n-1} \backslash\left(\operatorname{Dom}\left(M,\left(C_{3.4}(K+R)\right)^{-4}\right) \cup \operatorname{Comp}(M, \rho)\right):\right. & \\
& \left.z_{\text {small }}=w_{1}+\mathrm{i} w_{2}, w_{1} \perp w_{2},\left\|w_{1}\right\|_{2} \geq\left\|w_{2}\right\|_{2}\right\}
\end{aligned}
$$

instead of $S_{\mathbb{C}}^{n-1} \backslash\left(\operatorname{Dom}\left(M,\left(C_{3.4}(K+R)\right)^{-4}\right) \cup \operatorname{Comp}(M, \rho)\right)$. Therefore our revised goal is to show that the set of genuinely complex vectors, when specialized to $\mathcal{Z}$, admits a net of small cardinality. 
To this end, fixing a set $J \subset[n]$, we start with constructing a small net for the set of pairs $(\phi, \psi)$ with $\phi \perp \psi$ in the unit sphere of $\mathbb{R}^{J} \times \mathbb{R}^{J}$ for which the value of the two-dimensional LCD, the auxiliary parameter $\Delta(\cdot)$, and the de-correlation $d(\phi, \psi)=\|\phi\|_{2}\|\psi\|_{2}$ are approximately constant. The condition on the two-dimensional LCD means that there exists a linear combination of the vectors $\phi$ and $\psi$ which is close to an integer point. Our aim is to use this linear combination to construct separate approximations of $\phi$ and $\psi$.

For any $\gamma>0$, let us denote $\mathbb{Z}_{\gamma}^{J}:=\mathbb{Z}^{J} \cap \gamma B_{2}^{|J|}$. Using a simple volumetric comparison argument we have following estimate on $\left|\mathbb{Z}_{\gamma}^{J}\right|$ :

$$
\left|\mathbb{Z}_{\gamma}^{J}\right| \leq\left(C_{0}\left(\frac{\gamma}{\sqrt{|J|}}+1\right)\right)^{|J|}
$$

for some absolute constant $C_{0}$. The main technical result of this section is the following lemma.

Lemma 4.6. Let $d \in(0,1)$, and $0<\alpha \leq d \mathfrak{D} \leq \Delta \leq \mathfrak{D}$. Define the set

$$
\begin{aligned}
S_{J}(\mathfrak{D}, \Delta, d):= & \left\{(\phi, \psi) \in \mathbb{R}^{J} \times \mathbb{R}^{J}: \phi \perp \psi,\|\phi\|_{2} \in[1 / 2,1],\|\psi\|_{2} \in[d, 3 d]\right. \\
& \exists \zeta \in \mathbb{R}^{2}\|\zeta\|_{2} \in[\mathfrak{D}, 2 \mathfrak{D}],\left\|\zeta_{1} \phi+\zeta_{2} \psi\right\|_{2} \in[\Delta, 2 \Delta], \\
& \text { and } \left.\operatorname{dist}\left(\zeta_{1} \phi+\zeta_{2} \psi, \mathbb{Z}^{J}\right)<\alpha\right\}
\end{aligned}
$$

Then, there exists a $\left(\frac{C_{4.5^{\alpha}}}{\mathfrak{D}}\right)$-net $\mathcal{M}_{J}(\mathfrak{D}, \Delta, d) \subset S_{J}(\mathfrak{D}, \Delta, d)$ such that

$$
\left|\mathcal{M}_{J}(\mathfrak{D}, \Delta, d)\right| \leq\left(\bar{C}_{4.5} \frac{d \mathfrak{D}^{2}}{\alpha} \cdot\left(\frac{1}{\sqrt{|J|}}+\frac{1}{\Delta}\right)\right)^{|J|} \cdot\left(\frac{\mathfrak{D}}{\alpha}\right)^{2},
$$

for some absolute constants $C_{4.5}$, and $\bar{C}_{4.5}$.

This lemma provides a significant improvement over the standard volumetric estimate yielding $\left(c \mathfrak{D}^{2} / \alpha^{2}\right)^{|J|}$. This improved bound precisely balances the term appearing in the small ball probability estimate. Note that the bounds on $\|\phi\|_{2}$ and $\|\psi\|_{2}$ imply that the de-correlation $d(\phi, \psi)$ is approximately constant in the set $S_{J}(\mathfrak{D}, \Delta, d)$, whereas the bounds on $\|\zeta\|_{2}$ and $\operatorname{dist}\left(\zeta_{1} \phi+\zeta_{2} \psi, \mathbb{Z}^{J}\right)$ ensure that the two-dimensional LCD and the auxiliary parameter $\Delta(\cdot)$ are approximately constant. Lemma 4.6 deals with the case when the de-correlation between $\phi$ and $\psi$ is relatively large, represented by the assumption $d \geq \alpha / \mathfrak{D}$, which in turn implies that the angle between the real and the imaginary part of the vectors is non-negligible. In Section 5 we use this criterion to formally define the notion of genuinely complex vectors.

We also point out to the reader that a net for the genuinely complex vectors was constructed in [39] (see Lemma 11.2 there). Using that net and the bound on the Lévy concentration function (see [39, Theorem 10.3]) it was then showed that there are no vectors with LCD less than $O(n)$ in the kernel of the matrix in context, with high probability. Repeating the same argument here one can 
at best hope to show that there does not exist any vector in $\operatorname{Ker}\left(B^{D}\right)$ with LCD $O(n p)$, with high probability. To treat the remaining vectors again one needs to apply bounds on Lévy concentration function (for example, see the bound derived in Proposition 5.3). However, for such vectors the bound is too weak to deduce almost sure convergence of the ESD of $\frac{1}{\sqrt{n p}} A_{n}$.

Hence, we need to proceed differently. In particular, we use all the parameters $D, \Delta$, and $d$ to find a net of appropriate size such that its cardinality balances with the small ball probability derived in Proposition 5.5 so that we are able to show that there are no vectors in $\operatorname{Ker}\left(B^{D}\right)$ with LCD less than $\exp \left(O\left(n p \rho^{4}\right)\right)$ with high probability.

Proof of Lemma 4.6. Assume that there exists $\zeta:=\left(\zeta_{1}, \zeta_{2}\right) \in \mathbb{R}^{2}$ and $q \in \mathbb{Z}^{J}$ satisfying

$$
\left\|\zeta_{1} \phi+\zeta_{2} \psi\right\|_{2} \in[\Delta, 2 \Delta] \text { and }\left\|\zeta_{1} \phi+\zeta_{2} \psi-q\right\|_{2}<\alpha .
$$

We consider two cases depending on the size of $\zeta_{1}$. Let us start with the case when this value is small. Consider the set

$$
\begin{gathered}
S_{J}^{0}(\mathfrak{D}, \Delta, d):=\left\{(\phi, \psi) \in S_{J}(\mathfrak{D}, \Delta, d): \exists\left(\zeta_{1}, \zeta_{2}\right) \in \mathbb{R}^{2},\left\|\left(\zeta_{1}, \zeta_{2}\right)\right\|_{2} \in[\mathfrak{D}, 2 \mathfrak{D}],\right. \\
\left|\zeta_{1}\right| \leq \frac{1}{2} d \mathfrak{D},\left\|\zeta_{1} \phi+\zeta_{2} \psi\right\|_{2} \in[\Delta, 2 \Delta], \text { and } \exists q \in \mathbb{Z}^{J} \text { such that } \\
\left.\left\|\zeta_{1} \phi+\zeta_{2} \psi-q\right\|_{2}<\alpha\right\} .
\end{gathered}
$$

Since $d<1$, note that the condition on $\zeta_{1}$ implies that $\mathfrak{D} / 2 \leq\left|\zeta_{2}\right| \leq 2 \mathfrak{D}$. Hence

$$
\Delta \leq\left\|\zeta_{1} \phi+\zeta_{2} \psi\right\|_{2} \leq \frac{1}{2} d \mathfrak{D}\|\phi\|_{2}+2 \mathfrak{D}\|\psi\|_{2} \leq 7 d \mathfrak{D} .
$$

We will approximate $\phi$ using the standard volumetric net and use (4.6) to construct a small net for $\psi$. To this end, consider $(\phi, \psi) \in S_{J}^{0}(\mathfrak{D}, \Delta, d)$ and let $\left(\zeta_{1}, \zeta_{2}\right) \in \mathbb{R}^{2}$ be the corresponding vector (i.e. for which (4.6) holds). Then, by the triangle inequality,

$$
\|q\|_{2}<\alpha+2 \Delta \leq 3 \Delta
$$

i.e. $q \in \mathbb{Z}_{3 \Delta}^{J}$. Denote by $\mathcal{N}_{\phi}$ an $(\alpha / \mathfrak{D})$-net in $B_{2}^{J}$ with

$$
\left|\mathcal{N}_{\phi}\right| \leq\left(\frac{3 \mathfrak{D}}{\alpha}\right)^{|J|} .
$$

Choose $\phi^{\prime} \in \mathcal{N}_{\phi}$ such that $\left\|\phi-\phi^{\prime}\right\|_{2}<\alpha / \mathfrak{D}$. Then

$$
\left\|\zeta_{1} \phi^{\prime}+\zeta_{2} \psi-q\right\|_{2}<\alpha+\left|\zeta_{1}\right| \cdot\left\|\phi-\phi^{\prime}\right\|_{2}<2 \alpha,
$$

as $\left|\zeta_{1}\right| \leq \frac{1}{2} d \mathfrak{D} \leq \mathfrak{D}$. Therefore

$$
\left\|\psi+\frac{\zeta_{1}}{\zeta_{2}} \phi^{\prime}-\frac{\mathfrak{D} / 2}{\zeta_{2}} \cdot \frac{q}{\mathfrak{D} / 2}\right\|_{2}<\frac{2 \alpha}{\left|\zeta_{2}\right|}<\frac{4 \alpha}{\mathfrak{D}} .
$$


We observe that

$$
\left|\frac{\zeta_{1}}{\zeta_{2}}\right| \bigvee\left|\frac{\mathfrak{D} / 2}{\zeta_{2}}\right| \leq 1, \quad\left\|\phi^{\prime}\right\|_{2} \leq 1, \quad \text { and } \quad\left\|\frac{q}{\mathfrak{D} / 2}\right\|_{2} \leq 6 \frac{\Delta}{\mathfrak{D}} \leq 6
$$

where the last inequality follows from our assumption $\Delta \leq \mathfrak{D}$. Next let $\mathcal{N}_{\square}$ be an $(\alpha / \mathfrak{D})$-net in the unit square in $\mathbb{R}^{2}$ with $\left|\mathcal{N}_{\square}\right| \leq(6 \mathfrak{D} / \alpha)^{2}$. Using (4.8), and applying the triangle inequality, we now see that there exists $\left(x_{1}, x_{2}\right) \in \mathcal{N}_{\square}$ such that

$$
\left\|\psi-x_{1} \phi^{\prime}-x_{2} \frac{q}{\mathfrak{D} / 2}\right\|_{2}<\frac{11 \alpha}{\mathfrak{D}} .
$$

Hence,

$$
\mathcal{M}_{J}^{0}(\mathfrak{D}, \Delta, d):=\left\{\left(\phi^{\prime}, x_{1} \phi^{\prime}+x_{2} \frac{q}{\mathfrak{D} / 2}\right): \phi^{\prime} \in \mathcal{N}_{\phi}, q \in \mathbb{Z}_{3 \Delta}^{J},\left(x_{1}, x_{2}\right) \in \mathcal{N}_{\square}\right\},
$$

is a $\frac{12 \alpha}{\mathfrak{D}}$-net of $S_{J}^{0}(\mathfrak{D}, \Delta, d)$, with

$$
\begin{aligned}
\left|\mathcal{M}_{J}^{0}(\mathfrak{D}, \Delta, d)\right| \leq\left|\mathcal{N}_{\phi}\right| \cdot\left|\mathbb{Z}_{3 \Delta}^{J}\right| & \cdot\left|\mathcal{N}_{\square}\right| \leq\left(\frac{3 C_{0} \mathfrak{D}}{\alpha} \cdot\left(\frac{3 \Delta}{\sqrt{|J|}}+1\right)\right)^{|J|} \cdot\left(\frac{6 \mathfrak{D}}{\alpha}\right)^{2} \\
& \leq\left(63 C_{0} \frac{d \mathfrak{D}^{2}}{\alpha} \cdot\left(\frac{1}{\sqrt{|J|}}+\frac{1}{\Delta}\right)\right)^{|J|} \cdot\left(\frac{6 \mathfrak{D}}{\alpha}\right)^{2}
\end{aligned}
$$

where (4.5) has been used to bound $\left|\mathbb{Z}_{3 \Delta}^{J}\right|$ and (4.7) has been used to replace $\Delta$ by $d \mathfrak{D}$ in the last inequality.

Turning to prove the case of $\left|\zeta_{1}\right|>\frac{1}{2} d \mathfrak{D}$ we denote

$$
S_{J}^{1}(\mathfrak{D}, \Delta, d):=S_{J}(\mathfrak{D}, \Delta, d) \backslash S_{J}^{0}(\mathfrak{D}, \Delta, d) .
$$

That is,

$$
\begin{gathered}
S_{J}^{1}(\mathfrak{D}, \Delta, d):=\left\{(\phi, \psi) \in S_{J}(\mathfrak{D}, \Delta, d): \exists\left(\zeta_{1}, \zeta_{2}\right) \in \mathbb{R}^{2},\left\|\left(\zeta_{1}, \zeta_{2}\right)\right\|_{2} \in[\mathfrak{D}, 2 \mathfrak{D}],\right. \\
\left|\zeta_{1}\right| \in\left[\frac{1}{2} d \mathfrak{D}, 2 \mathfrak{D}\right],\left\|\zeta_{1} \phi+\zeta_{2} \psi\right\|_{2} \in[\Delta, 2 \Delta] \text { and } \exists q \in \mathbb{Z}^{J} \text { such that } \\
\left.\left\|\zeta_{1} \phi+\zeta_{2} \psi-q\right\|_{2}<\alpha\right\} .
\end{gathered}
$$

Now let us construct a net in $S_{J}^{1}(\mathfrak{D}, \Delta, d)$. Our strategy here is opposite to what we used in the previous case. Namely, we use the volumetric approximation for $\psi$ and then use (4.6) to approximate $\phi$. To this end, consider any $(\phi, \psi) \in$ $S_{J}^{1}(\mathfrak{D}, \Delta, d)$ and let $\left(\zeta_{1}, \zeta_{2}\right) \in \mathbb{R}^{2}$ be the corresponding vector. As in the previous case we see $\|q\|_{2}<3 \Delta$, i.e. $q \in \mathbb{Z}_{3 \Delta}^{J}$. Since $\left|\zeta_{1}\right| \geq \frac{1}{2} d \mathfrak{D}$ and $\left|\zeta_{2}\right| \leq 2 \mathfrak{D}$ we also see that $24\left\|\zeta_{1} \phi\right\|_{2} \geq 6 d \mathfrak{D} \geq\left\|\zeta_{2} \psi\right\|_{2}$. Therefore

$$
\Delta \leq\left\|\zeta_{1} \phi\right\|_{2}+\left\|\zeta_{2} \psi\right\|_{2} \leq 25\left\|\zeta_{1} \phi\right\|_{2} \leq 25\left|\zeta_{1}\right| .
$$


Recall that by assumption, $\alpha / \mathfrak{D} \leq d$. Hence, we see that

$$
\left|\mathcal{N}_{\psi}\right| \leq\left(\frac{9 d \mathfrak{D}}{\alpha}\right)^{|J|}
$$

where $\mathcal{N}_{\psi}$ is an $(\alpha / \mathfrak{D})$-net in $3 d B_{2}^{J}$. Since $\|\psi\|_{2} \leq 3 d$, there exists $\psi^{\prime} \in \mathcal{N}_{\psi}$ such that $\left\|\psi-\psi^{\prime}\right\|_{2}<\alpha / \mathfrak{D}$. As in the previous case, this yields

$$
\left\|\zeta_{1} \phi+\zeta_{2} \psi^{\prime}-q\right\|_{2}<\alpha+\left|\zeta_{2}\right| \cdot\left\|\psi-\psi^{\prime}\right\|_{2} \leq 3 \alpha
$$

and so

$$
\left\|\phi+\frac{\Delta \zeta_{2}}{50 \mathfrak{D} \zeta_{1}} \cdot \frac{50 \mathfrak{D} \psi^{\prime}}{\Delta}-\frac{\Delta}{25 \zeta_{1}} \cdot \frac{25 q}{\Delta}\right\|_{2}<\frac{3 \alpha}{\left|\zeta_{1}\right|} \leq \frac{75 \alpha}{\Delta},
$$

where we have used (4.9) in the last step. Note that

$$
\left|\frac{\Delta \zeta_{2}}{50 \mathfrak{D} \zeta_{1}}\right| \bigvee\left|\frac{\Delta}{25 \zeta_{1}}\right| \leq 1, \quad\left\|\frac{50 \mathfrak{D} \psi^{\prime}}{\Delta}\right\|_{2} \leq \frac{150 d \mathfrak{D}}{\Delta} \leq 150, \quad \text { and } \quad\left\|\frac{25 q}{\Delta}\right\|_{2} \leq 75
$$

Let $\mathcal{N}_{\square}$ be the same $(\alpha / \mathfrak{D})$-net in the unit square as in the previous case. Since $\Delta \leq \mathfrak{D}$, combining the previous estimates with the triangle inequality, we have that there exists a $\left(x_{1}, x_{2}\right) \in \mathcal{N}_{\square}$ such that

$$
\left\|\phi-x_{1} \cdot \frac{50 \mathfrak{D} \psi^{\prime}}{\Delta}-x_{2} \cdot \frac{25 q}{\Delta}\right\|_{2}<\frac{300 \alpha}{\Delta}
$$

Using the fact $\Delta \leq \mathfrak{D}$ again, we now obtain an $(\alpha / \mathfrak{D})$-net $\mathcal{M}_{\phi}$ in $\left(\frac{300 \alpha}{\Delta}\right) \cdot B_{2}^{J}$ with

$$
\left|\mathcal{M}_{\phi}\right| \leq\left(\frac{900 \mathfrak{D}}{\Delta}\right)^{|J|}
$$

Thus there exists $\nu \in \mathcal{M}_{\phi}$ such that

$$
\left\|\phi-x_{1} \cdot \frac{50 \mathfrak{D} \psi^{\prime}}{\Delta}-x_{2} \cdot \frac{25 q}{\Delta}-\nu\right\|_{2}<\frac{\alpha}{\mathfrak{D}} .
$$

This implies that the set

$$
\begin{aligned}
& \mathcal{M}_{J}^{1}(\mathfrak{D}, \Delta, d):=\left\{\left(x_{1} \cdot \frac{50 \mathfrak{D} \psi^{\prime}}{\Delta}+x_{2} \cdot \frac{25 q}{\Delta}+\nu, \psi^{\prime}\right):\right. \\
&\left.\psi^{\prime} \in \mathcal{N}_{\psi}, q \in \mathbb{Z}_{3 \Delta}^{\gamma}, \nu \in \mathcal{M}_{\phi},\left(x_{1}, x_{2}\right) \in \mathcal{N}_{\square}\right\}
\end{aligned}
$$

is a $(2 \alpha / \mathfrak{D})$-net for $S_{J}^{1}(\mathfrak{D}, \Delta, d)$. We observe that

$$
\begin{aligned}
\left|\mathcal{M}_{J}^{1}(\mathfrak{D}, \Delta, d)\right| \leq & \left|\mathcal{N}_{\psi}\right| \cdot\left|\mathbb{Z}_{3 \Delta}\right| \cdot\left|\mathcal{M}_{\phi}\right| \cdot\left|\mathcal{N}_{\square}\right| \\
& \leq\left(\bar{C} \frac{d \mathfrak{D}}{\alpha} \cdot\left(\frac{\Delta}{\sqrt{|J|}}+1\right) \cdot \frac{\mathfrak{D}}{\Delta}\right)^{|J|} \cdot\left(\frac{\mathfrak{D}}{\alpha}\right)^{2} \\
& \leq\left(\bar{C} \frac{d \mathfrak{D}^{2}}{\alpha} \cdot\left(\frac{1}{\sqrt{|J|}}+\frac{1}{\Delta}\right)\right)^{|J|} \cdot\left(\frac{\mathfrak{D}}{\alpha}\right)^{2},
\end{aligned}
$$


where $\bar{C}$ is some absolute constant.

Since $S_{J}(\mathfrak{D}, \Delta, d)=S_{J}^{0}(\mathfrak{D}, \Delta, d) \cup S_{J}^{1}(\mathfrak{D}, \Delta, d)$, it therefore means that

$$
\mathcal{M}_{J}(\mathfrak{D}, \Delta, d):=\mathcal{M}_{J}^{0}(\mathfrak{D}, \Delta, d) \cup \mathcal{M}_{J}^{1}(\mathfrak{D}, \Delta, d)
$$

is a $(C \alpha / \mathfrak{D})$-net for the set $S_{J}(\mathfrak{D}, \Delta, d)$, where $C$ is an absolute constant.

The net $\mathcal{M}_{J}(\mathfrak{D}, \Delta, d)$ constructed above is not necessarily contained in $S_{J}(\mathfrak{D}, \Delta, d)$. However, we can construct a new net by replacing each point of this net by a point of the set $S_{J}(\mathfrak{D}, \Delta, d)$ which is within distance $C \alpha / \mathfrak{D}$ from this point. If a $(C \alpha / \mathfrak{D})$-close point does not exist, we skip the original point. Such process creates a $(2 C \alpha / \mathfrak{D})$-net contained in $S_{J}(\mathfrak{D}, \Delta, d)$ without increasing the cardinality. Thus the lemma is proved.

Building on Lemma 4.6 we now obtain a net with small cardinality for the collection of vectors $z$ for which $D_{2}\left(z_{\text {small }} /\left\|z_{\text {small }}\right\|_{2}\right) \approx \mathfrak{D}, \Delta\left(z_{\text {small }} /\left\|z_{\text {small }}\right\|_{2}\right) \approx$ $\Delta$, and $d\left(z_{\text {small }} /\left\|z_{\text {small }}\right\|_{2}\right) \approx d$, where we recall that the vector $z_{\text {small }}$ contains $n-M>n / 2$ coordinates of $z$ having the smallest magnitude. To this end, let us define the following set:

$$
\begin{aligned}
& \mathcal{Z}(\mathfrak{D}, \Delta, d):=\left\{z \in \mathcal{Z}: D_{2}\left(z_{\text {small }} /\left\|z_{\text {small }}\right\|_{2}\right) \in[\mathfrak{D},(3 / 2) \mathfrak{D}]\right. \\
& \left.\Delta\left(z_{\text {small }} /\left\|z_{\text {small }}\right\|_{2}\right) \in[\Delta,(3 / 2) \Delta], d\left(z_{\text {small }} /\left\|z_{\text {small }}\right\|_{2}\right) \in[d,(3 / 2) d]\right\} .
\end{aligned}
$$

As will be seen in Section 5, the small ball probability for the images of such vectors is controlled by the values of the two-dimensional LCD and the realimaginary de-correlation. So we partition this set according to $D_{2}(\cdot), \Delta(\cdot)$, and $d(\cdot)$. The net $\mathcal{M}_{J}(\mathfrak{D}, \Delta, d)$ provides a net for the vectors which have $D_{2}(\cdot) \approx$ $\mathfrak{D}, \Delta(\cdot) \approx \Delta$, and $d(\cdot) \approx d$. This is shown in the proposition below.

Proposition 4.7. Let $d \in(0,1), \mathfrak{D}, \Delta>1$, and denote

$$
\alpha:=L \sqrt{\log _{1} \frac{\Delta}{2^{7} L}} .
$$

Assume that $\alpha \leq d \mathfrak{D} \leq \Delta \leq \mathfrak{D}$. Then there exist absolute constants $C_{4.7}, \bar{C}_{4.7}$, and $a \operatorname{set} \mathcal{N}(\mathfrak{D}, \Delta, d) \subset \mathcal{Z}(\mathfrak{D}, \Delta, d)$ with

$$
|\mathcal{N}(\mathfrak{D}, \Delta, d)| \leq \bar{C}_{4.7}^{n}\left(\frac{n}{\rho M} \cdot \frac{\mathfrak{D}}{\alpha}\right)^{5 M} \cdot\left(\frac{d \mathfrak{D}^{2}}{\alpha} \cdot\left(\frac{1}{\sqrt{n}}+\frac{1}{\Delta}\right)\right)^{n-M}
$$

having the following approximation properties: Let $z \in \mathcal{Z}(\mathfrak{D}, \Delta, d)$ be any vector and denote $J=\operatorname{small}(z)$. Then there exists $w \in \mathcal{N}(\mathfrak{D}, \Delta, d)$ such that

$$
\begin{array}{r}
\left\|\frac{z_{J}}{\left\|z_{J}\right\|_{2}}-\frac{w_{J}}{\left\|w_{J}\right\|_{2}}\right\|_{2}<C_{4.7} \frac{\alpha}{\mathfrak{D}}, \quad\left\|z_{J^{c}}-w_{J^{c}}\right\|_{2} \leq C_{4.7} \frac{\rho \alpha}{\mathfrak{D}}, \\
\quad\left|\left\|z_{J}\right\|_{2}-\left\|w_{J}\right\|_{2}\right| \leq C_{4.7} \frac{\rho \alpha}{\mathfrak{D}} .
\end{array}
$$


Remark 4.8. Note that Lemma 4.6 holds also for any subset of $S_{J}(\mathfrak{D}, \Delta, d)$. That is, given any $\mathcal{S} \subset S_{J}(\mathfrak{D}, \Delta, d)$ there exists a net $\mathcal{M}_{J}^{\mathcal{S}}(\mathfrak{D}, \Delta, d) \subset \mathcal{S}$ with the same properties as in Lemma 4.6. We use this version of Lemma 4.6 to prove Proposition 4.7. Similarly, we will see that given any $\mathcal{S} \subset \mathcal{Z}(\mathfrak{D}, \Delta, d)$ there exists a set $\mathcal{N}^{\mathcal{S}}(\mathfrak{D}, \Delta, d) \subset \mathcal{S}$ with the same approximation properties and the cardinality bound as in Proposition 4.7. This version of Proposition 4.7 will be used in Section 5 .

In proving Proposition 4.7 our strategy will be to use the net $\mathcal{M}_{J}^{\mathcal{S}}(\mathfrak{D}, \Delta, d)$, for some suitable choice of $\mathcal{S}$, obtained from Lemma 4.6, to approximate the small coordinates. The cardinality of the net to approximate the large ones will be obtained by a simple volumetric estimate.

Proof of Proposition 4.7. Fix a set $J \subset[n],|J|=n-M$, and denote

$$
\mathcal{Z}_{J}(\mathfrak{D}, \Delta, d):=\{z \in \mathcal{Z}(\mathfrak{D}, \Delta, d): \operatorname{small}(z)=J\} .
$$

Let us now construct an approximating set for this subset. Denote $\phi+\mathrm{i} v=$ $\phi(z)+\mathrm{i} \psi(z):=z_{J} /\left\|z_{J}\right\|_{2} \in \mathbb{C}^{J}$. Recalling the definition of $\Delta(\phi+\mathrm{i} \psi)$ we see that there exists $\zeta \in \mathbb{R}^{2}$ such that

$$
\begin{aligned}
\|\zeta\|_{2} & \leq(4 / 3) D_{2}(\phi+\mathrm{i} \psi) \leq 2 \mathfrak{D}, \quad\left\|\zeta_{1} \phi+\zeta_{2} \psi\right\|_{2} \leq(4 / 3) \Delta(\phi+\mathrm{i} \psi) \leq 2 \Delta \\
& \text { and } \operatorname{dist}\left(\zeta_{1} \phi+\zeta_{2} \psi, \mathbb{Z}^{n}\right)<L \sqrt{\log _{1} \frac{\left\|\zeta_{1} \phi+\zeta_{2} \psi\right\|_{2}}{2^{8} L}} \leq L \sqrt{\log _{1} \frac{\Delta}{2^{7} L}}=\alpha .
\end{aligned}
$$

Further recall that $d\left(z_{\text {small }} /\left\|z_{\text {small }}\right\|_{2}\right)=\|\phi\|_{2}\|\psi\|_{2}$ and note that by our convention we have $\|\phi\|_{2} \in[1 / 2,1]$. Thus we deduce that $\|\psi\|_{2} \in[d, 3 d]$. Hence $(\phi, \psi) \in S_{J}(\mathfrak{D}, \Delta, d)$, and in particular $(\phi, \psi) \in \mathcal{S}$ where $\mathcal{S}:=\{(\phi(z), \psi(z))$ : $z \in \mathcal{Z}(\mathfrak{D}, \Delta, d)\}$. So it can be approximated by an element of $\mathcal{M}_{J}^{\mathcal{S}}(\mathfrak{D}, \Delta, d)$. Set

$$
\mathcal{M}_{J}:=\left\{\phi+\mathrm{i} \psi:(\phi, \psi) \in \mathcal{M}_{J}^{\mathcal{S}}(\mathfrak{D}, \Delta, d)\right\} .
$$

Then for any $z \in \mathcal{Z}_{J}(\mathfrak{D}, \Delta, d)$, there exists $w^{\prime} \in \mathcal{M}_{J}$ such that

$$
\left\|\frac{z_{J}}{\left\|z_{J}\right\|_{2}}-w^{\prime}\right\|_{2}<C_{4.5} \frac{\alpha}{\mathfrak{D}} .
$$

For the set $J^{c}$, we will use a net satisfying the volumetric estimate. Since $z \in$ $S_{\mathbb{C}}^{n-1}$, there exists a set $\mathcal{N}_{J^{c}}$ with

$$
\left|\mathcal{N}_{J^{c}}\right| \leq\left(\frac{3}{C_{4.5^{\rho}}} \cdot \frac{\mathfrak{D}}{\alpha}\right)^{2 M} .
$$

such that for every $z \in \mathcal{Z}_{J}(D, \Delta, d)$ there exists a $w_{J^{c}} \in \mathcal{N}_{J^{c}}$ for which

$$
\left\|z_{J^{c}}-w_{J^{c}}\right\|_{2} \leq C_{4.5} \frac{\rho \alpha}{\mathfrak{D}} .
$$


Finally consider a net $\mathcal{N}_{[0,1]}$ with $\left|\mathcal{N}_{[0,1]}\right| \leq 3 \mathfrak{D} /\left(C_{4.5} \rho \alpha\right)$ such that for every $z \in \mathcal{Z}_{J}(\mathfrak{D}, \Delta, d)$ there exists a $\rho^{\prime} \in[0,1]$ for which

$$
\left|\left\|z_{J}\right\|_{2}-\rho^{\prime}\right| \leq C_{4.5} \frac{\rho \alpha}{\mathfrak{D}} .
$$

Now let us define

$$
\mathcal{N}(\mathfrak{D}, \Delta, d):=\bigcup_{|J|=n-M}\left\{\rho^{\prime} w^{\prime}+w^{\prime \prime}: \rho^{\prime} \in \mathcal{N}_{[0,1]}, w^{\prime} \in \mathcal{M}_{J}, w^{\prime \prime} \in \mathcal{N}_{J^{c}}\right\} .
$$

Then for any $z \in \mathcal{Z}(\mathfrak{D}, \Delta, d)$ there exists a $w=w_{J}+w_{J^{c}} \in \mathcal{N}(\mathfrak{D}, \Delta, d)$ such that

$$
\begin{aligned}
\left\|\frac{z_{J}}{\left\|z_{J}\right\|_{2}}-\frac{w_{J}}{\left\|w_{J}\right\|_{2}}\right\|_{2}<C_{4.5} \frac{\alpha}{\mathfrak{D}}, \quad\left\|z_{J^{c}}-w_{J^{c}}\right\|_{2} & \leq C_{4.5} \frac{\rho \alpha}{\mathfrak{D}}, \\
& \left|\left\|z_{J}\right\|_{2}-\left\|w_{J}\right\|_{2}\right| \leq C_{4.5} \frac{\rho \alpha}{\mathfrak{D}} .
\end{aligned}
$$

The set $\mathcal{N}(\mathfrak{D}, \Delta, d)$ thus constructed may not be contained in $\mathcal{Z}(\mathfrak{D}, \Delta, d)$. However, as in the proof of Lemma 4.5 this can be rectified easily. It thus remains to bound the cardinality of $\mathcal{N}(\mathfrak{D}, \Delta, d)$. By Lemma 4.6 , we have

$$
\begin{aligned}
& |\mathcal{N}(\mathfrak{D}, \Delta, d)| \leq \sum_{|J|=n-M}\left|\mathcal{N}_{J^{c}}\right| \cdot\left|\mathcal{N}_{[0,1]}\right| \cdot\left|\mathcal{M}_{J}\right| \\
& \leq\left(\begin{array}{c}
n \\
M
\end{array}\right) \cdot\left(\frac{3}{C_{4.6} \rho} \cdot \frac{\mathfrak{D}}{\alpha}\right)^{2 M+1} \cdot\left(\bar{C}_{4.6} \frac{d \mathfrak{D}^{2}}{\alpha} \cdot\left(\frac{1}{\sqrt{n-M}}+\frac{1}{\Delta}\right)\right)^{n-M} \cdot\left(\frac{\mathfrak{D}}{\alpha}\right)^{2} .
\end{aligned}
$$

Since $1 \leq M<n / 2$ the required estimate follows from a straightforward calculation. This completes the proof.

\section{The structure of the kernel in the genuinely complex case}

In this section, our goal is to show that with high probability, any genuinely complex vector in $\operatorname{Ker}\left(B^{D}\right)$ has a large two-dimensional LCD. Before proceeding any further let us formally define the notion of genuinely complex vectors:

$$
\begin{aligned}
& \operatorname{Compl}(\mathcal{Z}):=\{z \in \mathcal{Z}: \\
& \left.d\left(z_{\text {small }} /\left\|z_{\text {small }}\right\|_{2}\right) \geq \frac{4 L}{D_{2}\left(z_{\text {small }} /\left\|z_{\text {small }}\right\|_{2}\right)} \sqrt{\log _{1} \frac{\Delta\left(z_{\text {small }} /\left\|z_{\text {small }}\right\|_{2}\right)}{2^{7} L}}\right\},
\end{aligned}
$$

where we recall the definition of $\mathcal{Z}$ from (4.4). Equipped with the notion of genuinely complex vectors we state the main result of this section. 
Theorem 5.1. Let $B^{D}, A_{n}, \rho, K, R, r$, and $r^{\prime}$ be as in Proposition 3.1. Then there exist constants $c_{5.1}, c_{5.1}^{\prime}$, depending only on $K, R, r$, and the fourth moment of $\left\{\xi_{i j}\right\}$, such that if $p$ satisfies the inequality

$$
c_{5.1} \rho^{5} p n>1
$$

then we have

$$
\begin{array}{r}
\mathbb{P}\left(\exists z \in \operatorname{Compl}(\mathcal{Z}) \cap \operatorname{Ker}\left(B^{D}\right): D_{2}\left(z_{\text {small }} /\left\|z_{\text {small }}\right\|_{2}\right) \leq \exp \left(c_{5.1}^{\prime} \frac{n}{M}\right),\right. \\
\left.\left\|A_{n}\right\| \leq K \sqrt{p n}\right) \leq \exp \left(-\bar{c}_{5.1} n p\right),
\end{array}
$$

where $M=C_{3.1} \rho^{-4} p^{-1}$ and $z_{\text {small }}$ is the smallest $(n-M)$ coordinates of $z$ in modulus.

The proof of Theorem 5.1 is carried out by the following two-fold argument. Using Proposition 4.7 we show that the subset of vectors in $\operatorname{Compl}(\mathcal{Z})$ that have a large value of $\Delta\left(z_{\text {small }} /\left\|z_{\text {small }}\right\|_{2}\right)$ admits a net of small cardinality. This observation together with an estimate on the small ball probability, obtained from [39, Theorem 7.5], yields the desired conclusion for vectors $z \in \operatorname{Compl}(\mathcal{Z})$ which possess a large value of $\Delta\left(z_{\text {small }} /\left\|z_{\text {small }}\right\|_{2}\right)$ (see Proposition 5.2). For the other case, we first show that such vectors, upon rotation, must have a dominated real part. Applying Proposition 3.1 we show that this is impossible with high probability, which finishes the proof of Theorem 5.1. The rest of this section is devoted to implementing this idea.

First let us consider the case of large $\Delta\left(z_{\text {small }} /\left\|z_{\text {small }}\right\|_{2}\right)$. For such vectors we prove that the following holds:

Proposition 5.2. Let $s \geq 1$. Define the set $\mathcal{Z}(s)$ by

$$
\mathcal{Z}(s):=\left\{z \in \operatorname{Compl}(\mathcal{Z}): \Delta\left(z_{\text {small }} /\left\|z_{\text {small }}\right\|_{2}\right) \geq s L\right\} .
$$

Let $A_{n}, B^{D}, K, R$, and $\rho$ be as in Theorem 5.1. Then there exist $s_{5.2}, r_{\star}>1$, and $c_{5.2}^{\prime}>0$, depending only on $K, R$, and the fourth moment of $\left\{\xi_{i j}\right\}$ such that for any $r_{\star}^{2} p^{-1} \leq M \leq \rho n$ we have,

$$
\begin{array}{r}
\mathbb{P}\left(\exists z \in \mathcal{Z}\left(s_{5.2}\right) \cap \operatorname{Ker}\left(B^{D}\right): D_{2}\left(z_{\text {small }} /\left\|z_{\text {small }}\right\|_{2}\right) \leq \exp \left(c_{\left.5.2^{\prime} / M\right)}\right.\right. \\
\text { and } \left.\left\|A_{n}\right\| \leq K \sqrt{p n}\right) \leq e^{-n} .
\end{array}
$$

To prove Proposition 5.2 we use bounds on Lévy concentration function. Using such bounds we show that for any vector $z \in \mathcal{Z}(s)$ the $\ell_{2}$ norm of $B^{D} z$ cannot be too small with large probability. From the net constructed in Section 4 it follows that $\mathcal{Z}(s)$ admits a net of small cardinality which enables us to take the union bound and complete the proof of Proposition 5.2.

As mentioned above to prove Proposition 5.2 we need to derive bounds on the small ball probability, in particular on Lévy concentration function. Before 
deriving the bounds such bounds we need to fix some notation. Let $z \in \mathbb{C}^{m}$ and $J \subset[m]$. Denote $z_{J}:=\left(z_{i}\right)_{i \in J} \in \mathbb{C}^{J}$ and $V_{J}:=V\left(z_{J}\right)$, where we recall that for any $z^{\prime}=x+\mathrm{i} y \in \mathbb{C}^{m^{\prime}}$ we define $V\left(z^{\prime}\right):=\left(\begin{array}{l}x^{\top} \\ y^{\top}\end{array}\right)$. Further denote the real-imaginary de-correlation of $V_{J}$ by

$$
d\left(V_{J}\right):=d\left(z_{J}\right)=\left(\operatorname{det}\left(V_{J} V_{J}^{\top}\right)\right)^{1 / 2} .
$$

This parameter, along with the LCD of $z_{J} /\left\|z_{J}\right\|_{2}$ controls the Lévy concentration function of $\sum_{j=1}^{m} \Xi_{j} z_{j}$, for a sequence of independent random variables $\left\{\Xi_{j}\right\}_{j \in[m]}$. Below is the desired estimate on the Lévy concentration function, which is a direct corollary of [39, Theorem 7.5].

Proposition 5.3. Fix any positive integer $m$ and let $\Xi:=\left(\Xi_{1}, \ldots, \Xi_{m}\right) \in$ $\mathbb{R}^{m}, \Xi_{j}:=\delta_{j} \xi_{j}, j=1, \ldots, m$, where $\delta_{1}, \ldots, \delta_{m}$ are i.i.d. $\operatorname{Ber}(p)$, and $\xi_{j}$ are i.i.d. random variables satisfying

$$
\mathcal{L}\left(\xi_{j}, 1\right) \leq 1-c_{1} \quad \text { and } \quad \mathbb{P}\left(\left|\xi_{j}\right|>C_{1}\right) \leq c_{1} / 2
$$

for some absolute constants $C_{1}$ and $c_{1} \in(0,1)$. Then for any $z \in \mathbb{C}^{m}, J \in[m]$ such that $z_{J} \neq 0$, and $\varepsilon>0$, we have

$$
\mathcal{L}\left(V \Xi, \varepsilon \sqrt{p}\left\|V_{J}\right\|\right) \leq \frac{C_{5.3}}{d\left(V_{J}\right) /\left\|V_{J}\right\|}\left(\varepsilon+\frac{1}{\sqrt{p} D_{2}\left(V_{J} /\left\|V_{J}\right\|\right)}\right)^{2}
$$

and if $\left\|\operatorname{Re}\left(z_{J}\right)\right\|_{2} \geq\left\|\operatorname{Im}\left(z_{J}\right)\right\|_{2}$, then

$$
\mathcal{L}\left(V \Xi, \varepsilon \sqrt{p}\left\|V_{J}\right\|\right) \leq \bar{C}_{5.3}\left(\varepsilon+\frac{1}{\sqrt{p} D_{1}\left(\operatorname{Re}\left(z_{J}\right)\right) /\left\|V_{J}\right\|}\right)
$$

where $V:=V(z), C_{5.3}$ and $\bar{C}_{5.3}$ are some constants, depending only on $c_{1}$ and $C_{1}$.

Remark 5.4. We point out to the reader that the definition of LCD in [39] and that of ours are slightly different from each other. For example, to define LCD in [39] the function $\log _{+}(x):=\max \{\log x, 0\}$ was used instead of $\log _{1}(\cdot)$. Moreover the constants appearing in front of $L$ are different (compare Definition 4.1 with [39, Definition 7.1]). However, upon investigating the proof of [39, Theorem 7.5] it becomes evident that the same proof can be carried out for the LCDs $D_{2}(\cdot)$ and $D_{1}(\cdot)$ to obtain the same estimates on the Lévy concentration function. It only changes the constant that appears in [39, Eqn. (7.3)]. Below we apply this version of [39, Theorem 7.5].

Proof of Proposition 5.3. As mentioned above the proof is a straightforward application of [39, Theorem 7.5]. Indeed, we note that $\mathcal{L}(V \Xi, t) \leq \mathcal{L}\left(V_{J} \Xi_{J}, t\right)$, for any $t>0$, where $\Xi_{J}:=\left(\Xi_{j}\right)_{j \in J}$. The assertion (5.3) implies that

$$
\mathcal{L}\left(\Xi_{j}, 1\right) \leq 1-p c_{1} \quad \text { and } \quad \mathbb{P}\left(\left|\Xi_{j}\right|>C_{1}\right) \leq p c_{1} / 2 .
$$


Since $L=\left(\delta_{0} p\right)^{-1 / 2}$ (see Remark 4.5), shrinking $\delta_{0}$ if necessary, the inequality (5.4) follows directly from [39, Theorem 7.5], applied with $m=2$. To prove (5.5), using the triangle inequality we further note that $\mathcal{L}\left(V_{J} \Xi_{J}, t\right) \leq \mathcal{L}\left(x_{J}^{\top} \Xi_{J}, t\right)$. Thus applying [39, Theorem 7.5], with $m=1$ we obtain (5.5).

Applying Proposition 5.3 and standard tensorization techniques we obtain the following result, the proof of which is omitted.

Proposition 5.5. Let $B^{D}$ be as in Proposition 3.1. Fix any $z \in \mathbb{C}^{n}$ and $J:=$ $\operatorname{small}(z)$ such that $z_{J} \neq 0$. Then for any $\varepsilon>0$, we have

$$
\mathcal{L}\left(B^{D} z, \varepsilon \sqrt{p(n-1)}\left\|z_{J}\right\|_{2}\right) \leq\left[\frac{C_{5.5}}{d\left(z_{J} /\left\|z_{J}\right\|_{2}\right)}\left(\varepsilon+\frac{1}{\sqrt{p} D_{2}\left(z_{J} /\left\|z_{J}\right\|_{2}\right)}\right)^{2}\right]^{n-1},
$$

and if $\left\|\operatorname{Re}\left(z_{J}\right)\right\|_{2} \geq\left\|\operatorname{Im}\left(z_{J}\right)\right\|_{2}$, then

$$
\mathcal{L}\left(B^{D} z, \varepsilon \sqrt{p(n-1)}\left\|z_{J}\right\|_{2}\right) \leq\left[\bar{C}_{5.5}\left(\varepsilon+\frac{1}{\sqrt{p} D_{1}\left(\operatorname{Re}\left(z_{J}\right) /\left\|z_{J}\right\|_{2}\right)}\right)\right]^{n-1},
$$

for some constants $C_{5.5}$, and $\bar{C}_{5.5}$, depending only on $\mathbb{E}\left|\xi_{i j}\right|$ and $\mathbb{E}\left(\xi_{i j}^{4}\right)$.

Remark 5.6. The inequality (5.8) provides bounds on Lévy concentration function based on one-dimensional LCD. It will be used later in Section 6 to treat essentially real vectors.

To prove Proposition 5.2 we also need the following elementary lower bound on the LCD of non-dominated vectors. Its proof follows from [39, Proposition 7.4] and the definition of dominated vectors.

Lemma 5.7. If $z \notin \operatorname{Dom}\left(\mathrm{M}, \alpha_{\star}\right)$ for some $M<n$ and $\alpha_{\star}>0$, then we have

$$
D_{2}\left(z_{\text {small }} /\left\|z_{\text {small }}\right\|_{2}\right) \geq \frac{\alpha_{\star} \sqrt{M}}{2} .
$$

We are now ready to prove Proposition 5.2.

Proof of Proposition 5.2. The set in question can be partitioned into the subsets of $\mathcal{Z}(\mathfrak{D}, \Delta, d)$ appearing in Section 4. Indeed, using Lemma 5.7 we note that for any $z \in \mathcal{Z}(s)$ we have $D_{2}\left(z_{\text {small }} /\left\|z_{\text {small }}\right\|_{2}\right) \geq \frac{1}{2} \alpha_{\star} \sqrt{M} \geq \frac{\alpha_{\star} r_{\star}}{2} p^{-1 / 2}$ for some $\alpha_{\star}>0$. Since $L=\left(\delta_{0} p\right)^{-1 / 2}$, choosing $r_{\star}$ sufficiently large, we therefore obtain that

$$
\left\{z \in \mathcal{Z}(s): D_{2}\left(z_{\text {small }} /\left\|z_{\text {small }}\right\|_{2}\right) \leq \exp \left(c^{\prime} n / M\right)\right\} \subset \bigcup_{\mathcal{D}, \Delta, d} \mathcal{Z}(\mathfrak{D}, \Delta, d) \cap \mathcal{Z}(s),
$$

where the union is taken over all $\mathfrak{D}=2^{k}, C_{\star} L \leq \mathfrak{D} \leq \exp \left(c^{\prime} n / M\right)$, for some large constant $C_{\star}$, and over all $\Delta=2^{m}, d=2^{-\ell}$ satisfying $d \mathfrak{D} \leq \Delta \leq \mathfrak{D}$. Also note that for any $z \in \operatorname{Compl}(\mathcal{Z})$ we have

$$
d\left(z_{\text {small }} /\left\|z_{\text {small }}\right\|_{2}\right) \geq \frac{4 L}{D_{2}\left(z_{\text {small }} /\left\|z_{\text {small }}\right\|_{2}\right)} \sqrt{\log _{1} \frac{\Delta\left(z_{\text {small }} /\left\|z_{\text {small }}\right\|_{2}\right)}{2^{7} L}} .
$$


If $z \in \mathcal{Z}(\mathfrak{D}, \Delta, d)$ we further have that $D_{2}\left(z_{\text {small }} /\left\|z_{\text {small }}\right\|_{2}\right) \leq 2 \mathfrak{D}, d\left(z_{\text {small }} /\left\|z_{\text {small }}\right\|_{2}\right) \leq$ $2 d$, and $\Delta\left(z_{\text {small }} /\left\|z_{\text {small }}\right\|_{2}\right) \geq \Delta$. Therefore, it follows from (5.9) that

$$
\alpha:=L \sqrt{\log _{1} \frac{\Delta}{2^{7} L}} \leq d \mathfrak{D} .
$$

So it allows us to use Proposition 4.7. Recalling that $M \geq r_{\star}^{2} p^{-1} \geq p^{-1}$, we see that the number of different values of $\mathfrak{D}$ appearing in the partitions is bounded by $c^{\prime} p n$. Using the fact that $\alpha \geq L$, we see that the number of different values of $d$ is bounded by the same number, and so is the number of different values of $\Delta$. Therefore, using the union bound, we deduce that it is enough to show that

$$
\mathbb{P}\left(\exists z \in \mathcal{Z}(D, \Delta, d) \cap \mathcal{Z}(s): B^{D} z=0, \text { and }\left\|B^{D}\right\| \leq(K+R) \sqrt{p n}\right) \leq e^{-2 n},
$$

for each such tripple $(\mathfrak{D}, \Delta, d)$.

To this end, we note that $\mathcal{Z}(\mathfrak{D}, \Delta, d) \cap \mathcal{Z}(s)$ admits a net $\mathcal{N}(\mathfrak{D}, \Delta, d) \subset$ $\mathcal{Z}(\mathfrak{D}, \Delta, d) \cap \mathcal{Z}(s)$. Therefore, from Proposition 5.5 it follows that

$$
\mathcal{L}\left(B^{D} w, \varepsilon \sqrt{p(n-1)}\left\|w_{\text {small }}\right\|_{2}\right) \leq\left[\frac{C_{5.5}}{d}\left(\varepsilon+\frac{1}{\sqrt{p} \mathfrak{D}}\right)^{2}\right]^{n-1},
$$

for any $w \in \mathcal{N}(\mathfrak{D}, \Delta, d)$ and $\varepsilon>0$. Set

$$
\varepsilon_{0}:=40 C_{4.7}(K+R) \frac{\alpha}{\mathfrak{D}} .
$$

Since $\alpha \geq L=\left(\delta_{0} p\right)^{-1 / 2} \geq p^{-1 / 2}$ and $K, R \geq 1$ we note that $\varepsilon_{0} \geq \frac{1}{\sqrt{p} \mathfrak{D}}$. Therefore

$$
\mathbb{P}\left(\left\|B^{D} w\right\|_{2} \leq \frac{\varepsilon_{0}}{2}\left\|w_{\mathrm{small}}\right\|_{2} \cdot \sqrt{p n}\right) \leq\left[\frac{C}{d}\left((K+R) \frac{\alpha}{\mathfrak{D}}\right)^{2}\right]^{n-1}
$$

for some positive constant $C$. Hence, by the union bound and applying Proposition 4.7

$$
\begin{aligned}
& \mathbb{P}\left(\exists w \in \mathcal{N}(\mathfrak{D}, \Delta, d):\left\|B^{D} w\right\|_{2} \leq \frac{\varepsilon_{0}}{2}\left\|w_{\text {small }}\right\|_{2} \cdot \sqrt{p n}\right) \\
\leq & |\mathcal{N}(\mathfrak{D}, \Delta, d)| \cdot\left[\frac{C}{d}\left((K+R) \frac{\alpha}{\mathfrak{D}}\right)^{2}\right]^{n-1} \\
\leq & {\left[\frac{C}{d}\left((K+R) \frac{\alpha}{\mathfrak{D}}\right)^{2}\right]^{n-1} \cdot \bar{C}_{4.7}^{n}\left(\frac{n}{\rho M} \cdot \frac{\mathfrak{D}}{\alpha}\right)^{5 M} \cdot\left(\frac{d \mathfrak{D}^{2}}{\alpha} \cdot\left(\frac{1}{\sqrt{n}}+\frac{1}{\Delta}\right)\right)^{n-M} . }
\end{aligned}
$$

Recalling the definition of $\alpha$ and using the inequalities $L \leq \alpha \leq d \mathfrak{D}$ and $\Delta \leq \mathfrak{D}$ we note that

$$
\frac{1}{d} \cdot\left(\frac{\alpha}{\mathfrak{D}}\right)^{2} \leq \frac{\alpha}{\mathfrak{D}}=\frac{L}{\mathfrak{D}} \sqrt{\log _{1} \frac{\Delta}{2^{7} L}} \leq \frac{L}{\mathfrak{D}} \sqrt{\log _{1} \frac{\mathfrak{D}}{2^{7} L}} \leq \frac{1}{2^{7}} \sqrt{\frac{2^{7} L}{\mathfrak{D}}},
$$


where the last step follows from the fact that $\log x \leq x$ for any $x \geq e$. As we have already noted that $\mathfrak{D} \geq C_{\star} L$, for some large $C_{\star}$, we can enlarge $C_{\star}$ further (i.e. we increase $\left.r_{\star}\right)$ so that $\frac{C}{d}\left((K+R) \frac{\alpha}{\mathfrak{D}}\right)^{2} \bar{C}_{4.7}<1$. This means that we can drop the term

$$
\left[\frac{C}{d}\left((K+R) \frac{\alpha}{\mathfrak{D}}\right)^{2}\right]^{M-1} \cdot \bar{C}_{4.7}^{M}<1
$$

in (5.12). Therefore, from (5.12) we obtain

$$
\mathbb{P}\left(\exists w \in \mathcal{N}(\mathfrak{D}, \Delta, d):\left\|B^{D} w\right\|_{2} \leq \frac{\varepsilon_{0}}{2}\left\|w_{\text {small }}\right\|_{2} \cdot \sqrt{p n}\right) \leq \exp (-\Gamma n),
$$

where

$\Gamma:=-\left(1-\frac{M}{n}\right) \cdot \log \left(\frac{C^{\prime}(K+R)^{2} \alpha}{\sqrt{n}}+\frac{C^{\prime}(K+R)^{2} \alpha}{\Delta}\right)-\frac{5 M}{n} \log \left(\frac{n}{\rho M} \cdot \frac{\mathfrak{D}}{\alpha}\right)$,

and $C^{\prime}:=C \cdot \bar{C}_{4.7}$. To finish the proof we need to show that $\Gamma \geq 2$.

Turning to proof of this, we recall that $\frac{n}{M} \geq \frac{1}{\rho}, \alpha \geq L \geq 1$ and $\mathfrak{D} \leq \exp \left(c^{\prime} \frac{n}{M}\right)$. So, choosing $c^{\prime}$ sufficiently small we obtain

$$
\frac{5 M}{n} \log \left(\frac{n}{\rho M} \cdot \frac{\mathfrak{D}}{\alpha}\right) \leq \frac{10 M}{n} \log \left(\frac{n}{M}\right)+\frac{5 M}{n} \log \mathfrak{D} \leq 20 .
$$

Next recall that $L=\left(\delta_{0} p\right)^{-1 / 2}$ and $\Delta \leq \mathfrak{D} \leq \exp \left(c^{\prime} n / M\right)$. So

$$
\alpha=L \sqrt{\log _{1} \frac{\Delta}{2^{7} L}} \leq \sqrt{\frac{c^{\prime} n}{\delta_{0} M p}} .
$$

Therefore, using the fact that $M<n / 2$ we obtain

$$
\Gamma \geq-\frac{1}{2} \cdot \log \left(\frac{C^{\prime}(K+R)^{2} \sqrt{c^{\prime}}}{\sqrt{\delta_{0} M p}}+\frac{C^{\prime}(K+R)^{2} \alpha}{\Delta}\right)-20 .
$$

We claim that by choosing $s$ to be a sufficiently large constant, we can guarantee that

$$
\frac{C^{\prime}(K+R)^{2} \alpha}{\Delta}<e^{-50}
$$

Thus choosing $c^{\prime}$ small enough and recalling that $M p \geq r_{\star}^{2}$, from claim (5.14) we see that $\Gamma \geq 2$, providing the required bound for the probability.

Now let us check our claim (5.14). Using the definition of $\alpha$, choosing $s_{5.2}$ sufficiently large, and using the fact that the function $f(x):=x^{-1} \sqrt{\log _{1} x}$ tends to 0 as $x \rightarrow \infty$, we note that

$$
e^{50} \frac{C^{\prime}(K+R)^{2} \alpha}{\Delta}=e^{50} C^{\prime}(K+R)^{2} \frac{L}{\Delta} \sqrt{\log _{1} \frac{\Delta}{2^{7} L}} \leq 1,
$$

for any $\Delta$ such that $\Delta \geq s_{5.2} L$. This proves the claim (5.14). 
Thus we have shown that for a sufficiently large value of $s_{5.2}$,

$$
\mathbb{P}\left(\exists w \in \mathcal{N}(\mathfrak{D}, \Delta, d):\left\|B^{D} w\right\|_{2} \leq \frac{\varepsilon_{0}}{2}\left\|w_{\text {small }}\right\|_{2} \cdot \sqrt{p n}\right) \leq \exp (-2 n) .
$$

To deduce (5.10) from (5.15) we simply use the property of the net $\mathcal{N}(\mathfrak{D}, \Delta, d)$. Indeed, let us assume that there exists a $z \in \mathcal{Z}\left(s_{5.2}\right) \cap \mathcal{Z}(\mathfrak{D}, \Delta, d)$ so that $B^{D} z=$ 0 . Denoting $J=\operatorname{small}(z)$, using Proposition 4.7 , and the triangle inequality we see that there exists a $w \in \mathcal{N}(\mathfrak{D}, \Delta, d)$ such that

$$
\begin{aligned}
& \left\|B^{D} w\right\|_{2}=\left\|B^{D}(w-z)\right\|_{2} \\
& \leq\left\|B^{D}\right\|_{2} \cdot\left(\left\|z_{J^{c}}-w_{J^{c}}\right\|_{2}+\left\|w_{J}\right\|_{2}\left\|\frac{z_{J}}{\left\|z_{J}\right\|_{2}}-\frac{w_{J}}{\left\|w_{J}\right\|_{2}}\right\|_{2}+\left|\left\|w_{J}\right\|-\left\|z_{J}\right\|_{2}\right|\right) \\
\leq & (K+R) \sqrt{n p} \cdot\left(2 C_{4.7} \frac{\rho \alpha}{\mathfrak{D}}+C_{4.7} \frac{\alpha}{\mathfrak{D}}\left\|w_{J}\right\|_{2}\right) \leq 3 C_{4.7}(K+R) \frac{\alpha}{\mathfrak{D}}\left\|w_{J}\right\|_{2} \sqrt{n p},
\end{aligned}
$$

where the last inequality follows from the fact that $w \in \mathcal{N}(\mathfrak{D}, \Delta, d) \subset \mathcal{Z}\left(s_{5.2}\right) \subset$ $\operatorname{Comp}(M, \rho)^{c}$. To complete the proof, let us show that $\left\|w_{J}\right\|_{2} \leq 4\left\|w_{\text {small }}\right\|_{2}$. Assume for a moment that the opposite inequality holds. Since $\mathfrak{D} \geq \frac{\alpha_{\star} r_{\star}}{2} p^{-1 / 2}$, and $L=\left(\delta_{0} p\right)^{-1 / 2}$, choosing a sufficiently large $r_{\star}$, we may assume that

$$
C_{4.7} \frac{\alpha}{\mathfrak{D}} \leq C_{4.7} \frac{L}{\mathfrak{D}} \sqrt{\log _{1} \frac{\mathfrak{D}}{2^{7} L}} \leq \frac{1}{8} .
$$

Denote $\operatorname{small}(w)=I$. Combining the estimate above and Proposition 4.7, we see that

$$
\begin{aligned}
\left\|z_{I}\right\|_{2} & \leq\left\|w_{I}\right\|_{2}+\left\|z_{I \cap J}-w_{I \cap J}\right\|_{2}+\left\|z_{I \backslash J}-w_{I \backslash J}\right\|_{2} \\
& \leq \frac{1}{4}\left\|w_{J}\right\|_{2}+\left\|z_{J}-w_{J}\right\|_{2}+\left\|z_{J^{c}}-w_{J c}\right\|_{2} \\
& \leq \frac{1}{2}\left\|z_{J}\right\|_{2}+\frac{1}{4}\left\|z_{J}\right\|_{2}+\frac{1}{8} \rho<\left\|z_{J}\right\|_{2},
\end{aligned}
$$

where we used $\left\|z_{J}\right\|_{2} \geq \rho$ in the last inequality. This contradicts the definition of $J$ as $J=\operatorname{small}(z)$ and proves the desired inequality $\left\|w_{J}\right\|_{2} \leq 4\left\|w_{\text {small }}\right\|_{2}$.

Recalling the definition of $\varepsilon_{0}$ in (5.11), we now deduce (5.10) from (5.15). This finishes the proof.

Using Proposition 5.2 we now complete the proof of Theorem 5.1. The final ingredient for the proof of Theorem 5.1 is a lower bound on the one-dimensional LCD, the proof of which follows from [44, Lemma 6.2].

Lemma 5.8. For any $x \in \mathbb{R}^{m}, D_{1}(x) \geq \frac{1}{2\|x\|_{\infty}}$.

Proof of Theorem 5.1. We first claim that

$$
\mathbb{P}\left(\exists z \in \operatorname{Ker}\left(B^{D}\right) \cap S_{\mathbb{C}}^{n-1}: \Delta\left(z_{\text {small }} /\left\|z_{\text {small }}\right\|_{2}\right) \leq \frac{\rho^{-1}}{4 \sqrt{p}}\right) \leq \exp \left(-\bar{c}_{3.1} n p\right) .
$$


The probability bound above would follow from Proposition 3.1 if for any such $z$, we find a number $\nu \in \mathbb{C},|\nu|=1$ such that the vector $\nu z_{\text {small }}$ has a dominated real part. To implement this idea and show (5.16), we fix $z \in \operatorname{Ker}\left(B^{D}\right) \cap S_{\mathbb{C}}^{n-1}$ and denote $z_{\text {small }} /\left\|z_{\text {small }}\right\|_{2}=\phi+\mathrm{i} \psi$, where $\phi, \psi \in \mathbb{R}^{J}, J=\operatorname{small}(z)$. Let $\theta=\left(\theta_{1}, \theta_{2}\right) \in \mathbb{R}^{2}$ be such that

$$
\operatorname{dist}\left(\theta_{1} \phi+\theta_{2} \psi, \mathbb{Z}^{J}\right)<L \sqrt{\log _{1} \frac{\left\|\theta_{1} \phi+\theta_{2} \psi\right\|_{2}}{2^{8} L}}
$$

and $\left\|\theta_{1} \phi+\theta_{2} \psi\right\|_{2} \leq 2 \Delta\left(z_{\text {small }} /\left\|z_{\text {small }}\right\|_{2}\right)$. Denote

$$
w:=\frac{\theta_{1}-\mathrm{i} \theta_{2}}{\left|\theta_{1}-\mathrm{i} \theta_{2}\right|} z
$$

Then $w \in \operatorname{Ker}\left(B^{D}\right) \cap S_{\mathbb{C}}^{n-1}$ and $w_{\text {small }}=z_{\text {small }}$. Therefore (5.17) implies that

$$
D_{1}\left(\operatorname{Re}\left(w_{\text {small }}\right) /\left\|\operatorname{Re}\left(w_{\text {small }}\right)\right\|_{2}\right) \leq 2 \Delta\left(z_{\text {small }} /\left\|z_{\text {small }}\right\|_{2}\right) .
$$

Upon applying Lemma 5.8 we find that

$$
\left\|\frac{\operatorname{Re}\left(w_{\text {small }}\right)}{\left\|\operatorname{Re}\left(w_{\text {small }}\right)\right\|_{2}}\right\|_{\infty} \geq\left[4 \Delta\left(\frac{z_{\text {small }}}{\left\|z_{\text {small }}\right\|_{2}}\right)\right]^{-1} \geq \rho p^{1 / 2} .
$$

Since $w \in \operatorname{Ker}\left(B^{D}\right) \cap S_{\mathbb{C}}^{n-1}$ the claim (5.16) follows from Proposition 3.1.

Next, recalling the fact that $L=\left(\delta_{0} p\right)^{-1 / 2}$, and shrinking $\rho$, if necessary, we obtain that

$$
s_{5.2} L<\frac{\rho^{-1}}{4 \sqrt{p}} .
$$

The desired result then follows upon combining Proposition 5.2 and (5.16).

\section{Construction of the net and the structure of the kernel in the essentially real case}

In this section, we consider the class of vectors whose real and imaginary parts are almost linearly dependent. Namely, we introduce the set of essentially real vectors $\operatorname{Real}(\mathcal{Z})$ defined by

$$
\operatorname{Real}(\mathcal{Z}):=\mathcal{Z} \backslash \operatorname{Compl}(\mathcal{Z}),
$$

where we recall the definitions of $\mathcal{Z}$ and $\operatorname{Compl}(\mathcal{Z})$ from (4.4) and (5.1) respectively. Having shown that there does not exist any vector in $\operatorname{Compl}(\mathcal{Z}) \cap$ $\operatorname{Ker}\left(B^{D}\right)$ such that its two-dimensional LCD is small, it remains to show the same for $\operatorname{Real}(\mathcal{Z}) \cap \operatorname{Ker}\left(B^{D}\right)$. For essentially real vectors, the real-imaginary de-correlation $d(\cdot)$ is very small which precludes using (5.7). Instead we have to rely on the probability bound obtained in (5.8), which depends on the onedimensional LCD. As the bound on $D_{1}(u)$ implies a much more rigid arithmetic structure than a bound on $D_{2}(u)$, construction of a net of $\operatorname{real}(\mathcal{Z})$ would be 
easier. To construct such a net we will follow the method of [39]. Before finding a net let us remind the reader that the definition of $\operatorname{Compl}(\mathcal{Z})$ and hence that of $\operatorname{Real}(\mathcal{Z})$, depends on the two-dimensional LCD (see (5.1)). Since the bound on Lévy concentration function, for vectors in $\operatorname{Real}(\mathcal{Z})$, depends on the onedimensional LCD, we need a result that connects $D_{1}(\cdot)$ with $D_{2}(\cdot)$. The lemma below does that job and this is the sole reason of introducing different powers of 2 in the definitions (4.1) and (4.2) (recall Remark 4.2).

Lemma 6.1. Fix $z \in \operatorname{Real}(\mathcal{Z})$ and let $z_{\text {small }} /\left\|z_{\text {small }}\right\|_{2}=: \phi+\mathrm{i} \psi$. Then $D_{1}(\phi) \leq$ $2 D_{2}\left(z_{\text {small }} /\left\|z_{\text {small }}\right\|_{2}\right)$. In particular, if $D_{2}\left(z_{\text {small }} /\left\|z_{\text {small }}\right\|_{2}\right) \leq \mathfrak{D}$ then $D_{1}(\phi) \leq$ $2 \mathfrak{D}$.

Proof. Let us denote $J=\operatorname{small}(z)$. Denoting $\mathfrak{D}=D_{2}(\phi+\mathrm{i} \psi)$, we see that there exists $\theta=\left(\theta_{1}, \theta_{2}\right) \in \mathbb{R}^{2}$ with $\|\theta\|_{2} \leq 2 \mathfrak{D}$ and $\left\|\phi \theta_{1}+\psi \theta_{2}\right\|_{2} \geq \Delta\left(z_{J} /\left\|z_{J}\right\|_{2}\right) / \sqrt{2}$, and $q \in \mathbb{Z}^{J}$ such that

$$
\left\|\theta_{1} \phi+\theta_{2} \psi-q\right\|_{2}<L \sqrt{\log _{1} \frac{\left\|\theta_{1} \phi+\theta_{2} \psi\right\|_{2}}{2^{8} L}} .
$$

Using the triangle inequality, and the facts that $\left|\theta_{2}\right| \leq\|\theta\|_{2},\|\phi\|_{2} \cdot\|\psi\|_{2}=$ $d\left(z_{J} /\left\|z_{J}\right\|_{2}\right)$, and $\|\phi\|_{2} \geq 1 / 2$, we also obtain

$$
\left\|\theta_{1} \phi+\theta_{2} \psi\right\|_{2} \leq\left\|\theta_{1} \phi\right\|_{2}+4 d\left(z_{J} /\left\|z_{J}\right\|_{2}\right) D_{2}\left(z_{J} /\left\|z_{J}\right\|_{2}\right) .
$$

Since $\phi+\mathrm{i} \psi \in \operatorname{Real}(\mathcal{Z})$ we further note that

$$
d\left(z_{J} /\left\|z_{J}\right\|_{2}\right) D_{2}\left(z_{J} /\left\|z_{J}\right\|_{2}\right) \leq 4 L \sqrt{\log _{1} \frac{\Delta\left(z_{J} /\left\|z_{J}\right\|_{2}\right)}{2^{7} L}}
$$

(see (5.1) and (6.1)). Therefore denoting

$$
\alpha_{0}:=L \sqrt{\log _{1} \frac{\left\|\theta_{1} \phi+\theta_{2} \psi\right\|_{2}}{2^{6} \sqrt{2} L}},
$$

from (6.2)-(6.3) we note that

$$
\left\|\theta_{1} \phi+\theta_{2} \psi-q\right\|_{2}<\alpha_{0} \leq L \sqrt{\log _{1} \frac{\left\|\theta_{1} \phi\right\|_{2}+16 \alpha_{0}}{2^{6} \sqrt{2} L}} .
$$

It is easy to check that

$$
s \leq \sqrt{\log _{1}(t+s / 4 \sqrt{2})}, s>0 \text { and } t \geq 0 \Rightarrow s \leq \sqrt{\log _{1}(\sqrt{2} t)} .
$$

Hence we deduce that

$$
\left\|\theta_{1} \phi+\theta_{2} \psi-q\right\|_{2}<L \sqrt{\log _{1} \frac{\left\|\theta_{1} \phi\right\|_{2}}{2^{6} L}} .
$$


As we have already noted $\left\|\theta_{2} \psi\right\|_{2} \leq 4 d\left(z_{J} /\left\|z_{J}\right\|_{2}\right) D_{2}\left(z_{J} /\left\|z_{J}\right\|_{2}\right)$, using the fact $z \in \operatorname{Real}(\mathcal{Z})$, the triangle inequality, and $(6.4)$, we conclude

$$
\left\|\theta_{1} \phi-q\right\|_{2} \leq\left\|\theta_{1} \phi+\theta_{2} \psi-q\right\|_{2}+\left\|\theta_{2} \psi\right\|_{2} \leq 17 \alpha_{0}<2^{5} L \sqrt{\log _{1} \frac{\left\|\theta_{1} \phi\right\|_{2}}{2^{6} L}} .
$$

Since $\left|\theta_{1}\right| \leq\|\theta\|_{2} \leq 2 \mathfrak{D}$, the proof of the lemma is now complete.

Next we find a small net for $\operatorname{Real}(\mathcal{Z})$. As in the genuinely complex case, we start with constructing a small net for the set of the small coordinates.

Lemma 6.2. Fix $J \subset[n]$ and $0<\widetilde{\alpha} \leq \mathfrak{D}$. Define

$$
\begin{aligned}
S_{J}(\mathfrak{D}) & :=\left\{(u, v) \in \mathbb{R}^{J} \times \mathbb{R}^{J}:\|u\|_{2}^{2}+\|v\|_{2}^{2}=1,\|u\|_{2} \geq\|v\|_{2},\right. \\
& \left.d(u, v) \leq \widetilde{\alpha} / \mathfrak{D}, \text { and } \exists \theta \in[\mathfrak{D}, 3 \mathfrak{D}], \text { such that } \operatorname{dist}\left(\theta u, \mathbb{Z}^{J}\right)<\widetilde{\alpha}\right\} .
\end{aligned}
$$

Then, there exists a $\left(\frac{C_{6.2^{\widetilde{\alpha}}}}{\mathfrak{D}}\right)$-net $\mathcal{M}_{J}(\mathfrak{D}) \subset S_{J}(\mathfrak{D})$ with

$$
\left|\mathcal{M}_{J}(\mathfrak{D})\right| \leq \frac{\mathfrak{D}}{\widetilde{\alpha}} \cdot\left(\bar{C}_{6.2}\left(\frac{\mathfrak{D}}{\sqrt{|J|}}+1\right)\right)^{|J|}
$$

where $C_{6.2}$ and $\bar{C}_{6.2}$ are some absolute constants.

Proof. Let $(u, v) \in S_{J}(\mathfrak{D})$, and let $\theta \in[\mathfrak{D}, 3 \mathfrak{D}], q \in \mathbb{Z}^{J}$ be such that

$$
\|\theta u-q\|_{2}<\widetilde{\alpha} .
$$

Then, using the triangle inequality, $\|q\|_{2}<\widetilde{\alpha}+|\theta| \leq 4 \mathfrak{D}$, and so $q \in \mathbb{Z}_{4 \mathfrak{D}}$. This implies that

$$
\left\|u-\frac{\mathfrak{D}}{\theta} \cdot \frac{q}{\mathfrak{D}}\right\|_{2}<\frac{\alpha}{\mathfrak{D}} \quad \text { where }\left|\frac{\mathfrak{D}}{\theta}\right| \leq 1,\left\|\frac{q}{\mathfrak{D}}\right\|_{2} \leq 4 .
$$

From the definition of real-imaginary de-correlation it also follows that

$$
\|v\|_{2} \leq 2 d(u, v) \leq \frac{2 \widetilde{\alpha}}{\mathfrak{D}}
$$

Let $\mathcal{N}_{1}$ be an $(\widetilde{\alpha} / \mathfrak{D})$-net in $[-1,1]$ with $\left|\mathcal{N}_{1}\right| \leq 2 \mathfrak{D} / \widetilde{\alpha}$. Define $\mathcal{M}_{J}^{1}(\mathfrak{D})$ by

$$
\mathcal{M}_{J}^{1}(\mathfrak{D}):=\left\{\left(x \frac{q}{\mathfrak{D}}, 0\right): q \in \mathbb{Z}_{4 \mathfrak{D}}, x \in \mathcal{N}_{1}\right\} .
$$

Then from (6.5)-(6.6) we deduce that $\mathcal{M}_{J}^{1}(\mathfrak{D})$ is a $(7 \widetilde{\alpha} / \mathfrak{D})$-net for $S_{J}(\mathfrak{D})$ and $\left|\mathcal{M}_{J}^{1}(\mathfrak{D})\right|=\left|\mathbb{Z}_{4 \mathfrak{D}}\right| \cdot\left|\mathcal{N}_{1}\right|$. This in combination with the bound in (4.5) yields the required estimate for the cardinality of the net. To complete the proof, we have to replace the constructed set of vectors by a subset of $S_{J}(\mathfrak{D})$. This is done in the same way as in Lemma 4.6. We skip the details. 
Now we use Lemma 6.2 to construct a small net in the set of essentially real vectors with an approximately constant value of the one-dimensional LCD. Define the set $\widetilde{\mathcal{Z}}(\mathfrak{D})$ by

$$
\begin{array}{r}
\widetilde{\mathcal{Z}}(\mathfrak{D}):=\left\{z \in \operatorname{Real}(\mathcal{Z}): \frac{z_{\text {small }}}{\left\|z_{\text {small }}\right\|_{2}}=\phi+\mathrm{i} \psi,\|\phi\|_{2} \geq\|\psi\|_{2}, D_{1}(\phi) \in[\mathfrak{D}, 2 \mathfrak{D}],\right. \\
d(\phi, \psi) \leq \widetilde{\alpha} / \mathfrak{D}\},
\end{array}
$$

where

$$
\widetilde{\alpha}:=2^{5} L \sqrt{\log _{1} \frac{\mathfrak{D}}{2^{5} L}},
$$

The set $\widetilde{\mathcal{Z}}(\mathfrak{D})$ is the collection of vectors in $\operatorname{Real}(\mathcal{Z})$ for which $D_{1}\left(z_{\text {small }} /\left\|z_{\text {small }}\right\|_{2}\right) \approx$ $\mathfrak{D}$. The condition $d(\phi, \psi) \leq \widetilde{\alpha} / \mathfrak{D}$ ensures that the real-imaginary de-correlation is small.

Proposition 6.3. Fix $\mathfrak{D}>1$. Let $\widetilde{\alpha}$ be as in (6.7) and assume $0<\widetilde{\alpha} \leq \mathfrak{D}$. Then there exist absolute constants $C_{6.3}, \bar{C}_{6.3}$, and a set $\widetilde{\mathcal{N}}(\mathfrak{D}) \subset \widetilde{\mathcal{Z}}(\mathfrak{D})$ with

$$
|\widetilde{\mathcal{N}}(\mathfrak{D})| \leq \bar{C}_{6.3}^{n}\left(\frac{n}{\rho M} \cdot \frac{\mathfrak{D}}{\widetilde{\alpha}}\right)^{4 M} \cdot\left(\frac{\mathfrak{D}}{\sqrt{n}}+1\right)^{n-M}
$$

having the following approximation property: Let $z \in \widetilde{\mathcal{Z}}(D)$ be any vector and denote $J=\operatorname{small}(z)$. Then there exists $w \in \tilde{\mathcal{N}}(D)$ such that

$$
\begin{aligned}
\left\|\frac{z_{J}}{\left\|z_{J}\right\|_{2}}-\frac{w_{J}}{\left\|w_{J}\right\|_{2}}\right\|_{2}<C_{6.3} \frac{\widetilde{\alpha}}{\mathfrak{D}}, \quad\left\|z_{J^{c}}-w_{J^{c}}\right\|_{2} & \leq C_{6.3} \frac{\rho \widetilde{\alpha}}{\mathfrak{D}}, \\
& \left|\left\|z_{J}\right\|_{2}-\left\|w_{J}\right\|_{2}\right| \leq C_{6.3} \frac{\rho \widetilde{\alpha}}{\mathfrak{D}} .
\end{aligned}
$$

Proposition 6.3 is derived from Lemma 6.2 in the same way as Proposition 4.7 was derived from Lemma 4.6. We omit the details.

Now, we are ready to prove the main result of this section which shows that with high probability, there are no essentially real vectors with a subexponential LCD in the kernel of $B^{D}$.

Proposition 6.4. Let $B^{D}, A_{n}, \rho, K, R, r$, and $r^{\prime}$ be as in Proposition 3.1. Then there exists a positive constant $c_{6.4}^{\prime}$, depending only on $K, R$, and the fourth moment of $\left\{\xi_{i j}\right\}$, such that

$$
\begin{array}{r}
\mathbb{P}\left(\exists z \in \operatorname{Real}(\mathcal{Z}) \cap \operatorname{Ker}\left(B^{D}\right): D_{2}\left(z_{\text {small }} /\left\|z_{\text {small }}\right\|_{2}\right) \leq \exp \left(c_{\left.6.4^{n} / M\right)}^{\prime}\right.\right. \\
\text { and } \left.\left\|A_{n}\right\| \leq K \sqrt{p n}\right) \leq e^{-n},
\end{array}
$$

where $M=C_{3.1} \rho^{-4} p^{-1}$. 
Proof. The proof of this proposition is very similar to that of Proposition 5.2. First we note that using Lemma 6.1 it follows that it is enough to show that, with high probability, there does not exist $z \in \operatorname{Ker}\left(B^{D}\right) \cap \operatorname{Real}(\mathcal{Z})$ such that $D_{1}(\phi(z)) \leq \exp \left(c^{\prime} n / M\right)$ for some small constant $c^{\prime}$, where $z_{\text {small }} /\left\|z_{\text {small }}\right\|_{2}=$ : $\phi(z)+\mathrm{i} \psi(z)$ with $\|\phi(z)\|_{2} \geq\|\psi(z)\|_{2}$. We then claim that the subset of $\operatorname{Real}(\mathcal{Z})$ in context can be partitioned into the sets $\widetilde{\mathcal{Z}}(\mathfrak{D})$ as follows:

$$
\left\{z \in \operatorname{Real}(\mathcal{Z}): D_{1}(\phi(z)) \leq \exp \left(c^{\prime} n / M\right)\right\} \subset \bigcup_{\mathfrak{D}} \widetilde{\mathcal{Z}}(\mathfrak{D})
$$

where the union is taken over all $\mathfrak{D}=2^{k}, \mathfrak{D} \leq \exp \left(c^{\prime} n / M\right)$. Note that the claim in (6.8) is obvious if we drop the requirement $d\left(z_{\text {small }} /\left\|z_{\text {small }}\right\|_{2}\right)=d(\phi(z), \psi(z)) \leq$ $\widetilde{\alpha} / \mathfrak{D}$ from the definition of $\widetilde{\mathcal{Z}}(\mathfrak{D})$. We show that the required condition on the real-imaginary de-correlation is automatically satisfied for all $z \in \operatorname{Real}(\mathcal{Z})$. Indeed, recalling the definition of $\operatorname{Real}(\mathcal{Z})$, and the fact that

$$
\Delta\left(z_{\text {small }} /\left\|z_{\text {small }}\right\|_{2}\right) \leq D_{2}\left(z_{\text {small }} /\left\|z_{\text {small }}\right\|_{2}\right)
$$

we see that for any $z \in \operatorname{Real}(\mathcal{Z})$,

$$
\begin{aligned}
& d\left(z_{\text {small }} /\left\|z_{\text {small }}\right\|_{2}\right) \leq \frac{4 L}{D_{2}\left(z_{\text {small }} /\left\|z_{\text {small }}\right\|_{2}\right)} \sqrt{\log _{1} \frac{D_{2}\left(z_{\text {small }} /\left\|z_{\text {small }}\right\|_{2}\right)}{2^{7} L}} \\
& \leq \frac{8 L}{D_{1}(\phi(z))} \sqrt{\log _{1} \frac{D_{1}(\phi(z))}{2^{8} L}},
\end{aligned}
$$

where the last inequality is obtained upon noting that $x \sqrt{\log _{1}(1 / x)}$ is an increasing function for $x \in\left(0, e^{-1}\right]$ together with an application of Lemma 6.1. If $z \in \operatorname{Real}(\mathcal{Z})$ such that $D_{1}(\phi(z)) \in[\mathfrak{D}, 2 \mathfrak{D}]$ then recalling the definition of $\widetilde{\alpha}$, from (6.9) we see that

$$
d(\phi(z), \psi(z))=d\left(z_{\text {small }} /\left\|z_{\text {small }}\right\|_{2}\right) \leq \widetilde{\alpha} / \mathfrak{D},
$$

which in turn proves the claim (6.8). We further claim that the lower bound on $\mathfrak{D}$ in $(6.8)$ can be improved to

$$
\mathfrak{D}_{0}:=C_{0} \rho^{-1} p^{-1 / 2},
$$

where $C_{0}:=\sqrt{C_{3.1}} / 2$. To see this we note that $\operatorname{Real}(\mathcal{Z}) \subset \operatorname{Incomp}(M, \rho)$. Therefore for any $z \in \operatorname{Real}(\mathcal{Z})$ we have

$$
\|\phi(z)\|_{\infty} \leq 2 \frac{\left\|z_{\text {small }}\right\|_{\infty}}{\left\|z_{\text {small }}\right\|_{2}} \leq \frac{2}{\rho \sqrt{M}}=\frac{2 \sqrt{p}}{\rho^{-1} \sqrt{C_{3.1}}}
$$

where the last step follows from our choice of $M$. Hence, using Lemma 5.8 we see that for any $z \in \operatorname{Real}(\mathcal{Z})$ we must have $D_{1}(\phi(z)) \geq \mathfrak{D}_{0}$. This establishes that the union in the RHS of (6.8) can be taken over all $\mathfrak{D}=2^{k}, \mathfrak{D}_{0} \leq \mathfrak{D} \leq \exp \left(c^{\prime} n / M\right)$. So using the union bound, we deduce that it is enough to show that

$$
\mathbb{P}\left(\exists z \in \widetilde{\mathcal{Z}}(\mathfrak{D}): B^{D} z=0 \text {, and }\left\|B^{D}\right\| \leq(K+R) \sqrt{p n}\right) \leq e^{-2 n} .
$$


for each such $\mathfrak{D}$.

To this end, using Proposition 5.5 we see that for any $w \in \tilde{\mathcal{N}}(\mathfrak{D})$ we have

$$
\mathcal{L}\left(B^{D} w, \varepsilon \sqrt{p(n-1)}\left\|w_{\text {small }}\right\|_{2}\right) \leq\left[\bar{C}_{5.5}\left(\varepsilon+\frac{1}{\sqrt{p} \mathfrak{D}}\right)\right]^{n-1} .
$$

Now set

$$
\widetilde{\varepsilon}_{0}:=40 C_{6.3}(K+R) \frac{\widetilde{\alpha}}{\mathfrak{D}}
$$

Since the fact $\widetilde{\alpha} \geq L=\left(\delta_{0} p\right)^{-1 / 2}$ implies that $\widetilde{\varepsilon}_{0} \geq \frac{1}{\sqrt{p} \mathfrak{D}}$, we obtain that for any $w \in \tilde{\mathcal{N}}(\mathfrak{D})$,

$$
\begin{aligned}
\mathbb{P}\left(\left\|B^{D} w\right\|_{2} \leq \frac{\varepsilon_{0}}{2}\left\|w_{\text {small }}\right\|_{2} \cdot \sqrt{p n}\right) \leq\left[\overline { C } _ { 5 . 5 } \left(\widetilde{\varepsilon}_{0}+\right.\right. & \left.\left.\frac{1}{\sqrt{p} \mathfrak{D}}\right)\right]^{n-1} \\
& \leq\left(\widetilde{C}(K+R) \frac{\widetilde{\alpha}}{\mathfrak{D}}\right)^{n-1},
\end{aligned}
$$

for some constant $\widetilde{C}$. Hence, by the union bound and applying Proposition 6.3 we obtain

$$
\begin{aligned}
\mathbb{P}\left(\exists w \in \tilde{\mathcal{N}}(\mathfrak{D}):\left\|B^{D} w\right\|_{2} \leq \frac{\varepsilon_{0}}{2}\left\|w_{\text {small }}\right\|_{2} \cdot \sqrt{p n}\right) \\
\leq|\widetilde{\mathcal{N}}(\mathfrak{D})| \cdot\left(\widetilde{C}(K+R) \frac{\widetilde{\alpha}}{\mathfrak{D}}\right)^{n-1} \\
\leq\left(C^{\prime}(K+R) \frac{\widetilde{\alpha}}{\mathfrak{D}}\right)^{n-1}\left(\frac{n}{\rho M} \cdot \frac{\mathfrak{D}}{\widetilde{\alpha}}\right)^{4 M}\left(\frac{\mathfrak{D}}{\sqrt{n}}+1\right)^{n-M},
\end{aligned}
$$

where $C^{\prime}$ is some large constant. Next recalling the definitions of $\widetilde{\alpha}$ and $\mathfrak{D}_{0}$, using the facts that $\mathfrak{D} \geq \mathfrak{D}_{0}, L=\left(\delta_{0} p\right)^{-1 / 2}$ and the function $f(x):=x \sqrt{\log _{1}(1 / x)}$ is increasing for $x \in\left(0, e^{-1}\right)$ we find that

$$
\frac{\widetilde{\alpha}}{\mathfrak{D}}=\frac{2^{5} L}{\mathfrak{D}} \sqrt{\log _{1} \frac{\mathfrak{D}}{2^{5} L}} \leq \frac{2^{5} L}{\mathfrak{D}_{0}} \sqrt{\log _{1} \frac{\mathfrak{D}_{0}}{2^{5} L}}=f\left(\frac{2^{5}}{C_{0} \rho^{-1} \delta_{0}^{1 / 2}}\right) .
$$

Recalling the definition of $C_{0}$ and enlarging $C_{3.1}$ we therefore note from above that we can assume $\widetilde{C}(K+R) \widetilde{\alpha} / \mathfrak{D}<1$. This yields

$$
\mathbb{P}\left(\exists w \in \widetilde{\mathcal{N}}(\mathfrak{D}):\left\|B^{D} w\right\|_{2} \leq \frac{\varepsilon_{0}}{2}\left\|w_{\text {small }}\right\|_{2} \cdot \sqrt{p n}\right) \leq \exp (-\widetilde{\Gamma} n),
$$

where

$$
\widetilde{\Gamma}:=-\left(1-\frac{M}{n}\right) \cdot \log \left(\frac{C^{\prime}(K+R) \widetilde{\alpha}}{\sqrt{n}}+\frac{C^{\prime}(K+R) \widetilde{\alpha}}{\mathfrak{D}}\right)-\frac{4 M}{n} \log \left(\frac{n}{\rho M} \cdot \frac{\mathfrak{D}}{\widetilde{\alpha}}\right) .
$$

We next show that $\widetilde{\Gamma} \geq 2$ which allows us to deduce that

$$
\mathbb{P}\left(\exists w \in \tilde{\mathcal{N}}(\mathfrak{D}):\left\|B^{D} w\right\|_{2} \leq \frac{\varepsilon_{0}}{2}\left\|w_{\text {small }}\right\|_{2} \cdot \sqrt{p n}\right) \leq \exp (-2 n) .
$$


To prove that $\widetilde{\Gamma} \geq 2$, we recall that $\frac{n}{M} \geq \frac{1}{\rho}$ and $L \leq \widetilde{\alpha} \leq \mathfrak{D} \leq \exp \left(c^{\prime} \frac{n}{M}\right)$. Therefore

$$
\frac{4 M}{n} \log \left(\frac{n}{\rho M} \cdot \frac{\mathfrak{D}}{\widetilde{\alpha}}\right) \leq 10,
$$

upon choosing $c^{\prime}$ sufficiently small. Using the fact $M \leq n / 2$, this yields

$$
\widetilde{\Gamma} \geq-\frac{1}{2} \cdot \log \left(\frac{C^{\prime}(K+R) \widetilde{\alpha}}{\sqrt{n}}+\frac{C^{\prime}(K+R) \widetilde{\alpha}}{\mathfrak{D}}\right)-10 .
$$

Recalling (6.10) we see that we may enlarge $C_{3.1}$ (and thus, the minimal value of $\mathfrak{D})$ further so that $\widetilde{C}(K+R) \widetilde{\alpha} / \mathfrak{D}<e^{-30}$. Using the upper bound for $\mathfrak{D}$, we also note that

$$
\frac{\widetilde{\alpha}}{\sqrt{n}} \leq \frac{2^{5} L \sqrt{\log _{1} \frac{\mathfrak{D}}{2^{5} L}}}{\sqrt{n}} \leq \frac{2^{5} L \sqrt{c^{\prime} \frac{n}{M}}}{\sqrt{n}}=\frac{2^{5} \sqrt{c^{\prime}}}{\sqrt{\delta_{0} M p}} \leq \frac{2^{5} \rho^{2} \sqrt{c^{\prime}}}{\sqrt{C_{3.1} \delta_{0}}} \leq e^{-30}
$$

where the second last inequality follows from our choice of $M$, and the last inequality results from enlarging $C_{3.1}$ once more. This completes the proof of the claim that $\widetilde{\Gamma} \geq 2$. Thus we have shown that (6.11) holds. The rest of the proof relies on the approximation of a general point of $\widetilde{\mathcal{Z}}(\mathfrak{D})$ by a point of the set $\widetilde{\mathcal{N}}(\mathfrak{D})$, and is exactly the same as that of Proposition 5.2. We leave the details to the reader. This completes the proof.

\section{Proof of Theorem 2.2}

In this section our goal is to combine the results of previous sections and finish the proof of Theorem 2.2. First let us state the following general result from which Theorem 2.2 follows.

Theorem 7.1. Let $A_{n}$ be an $n \times n$ matrix with i.i.d. entries $a_{i, j}=\delta_{i, j} \xi_{i, j}$, where $\left\{\delta_{i, j}\right\}$ are independent Bernoulli random variables taking value 1 with probability $p_{n} \in(0,1]$, and $\left\{\xi_{i, j}\right\}$ are i.i.d. centered real-valued random variables with unit variance and finite fourth moment. Fix $K, R \geq 1$, and $r \in(0,1]$ and let $\Omega_{K}:=\left\{\left\|A_{n}\right\| \leq K \sqrt{n p_{n}}\right\}$. Assume that $D_{n}$ is a diagonal matrix such that $\left\|D_{n}\right\| \leq R \sqrt{n p_{n}}$ and $\operatorname{Im}\left(D_{n}\right)=r^{\prime} \sqrt{n p_{n}} I_{n}$ with $\left|r^{\prime}\right| \in[r, 1]$. Then there exists constants $0<c_{7.1}, \bar{c}_{7.1}, c_{7.1}^{\prime}, C_{7.1}, C_{7.1}^{\prime}, \bar{C}_{7.1}<\infty$, depending only on $K, R, r$, and the fourth moment of $\left\{\xi_{i, j}\right\}$, such that for any $\varepsilon>0$ we have the following:

(i) If

$$
p_{n} \geq \frac{\bar{C}_{7.1} \log n}{n}
$$

then

$$
\begin{array}{r}
\mathbb{P}\left(\left\{s_{\min }\left(A_{n}+D_{n}\right) \leq c_{7.1} \varepsilon \exp \left(-C_{7.1} \frac{\log \left(1 / p_{n}\right)}{\log \left(n p_{n}\right)}\right) \sqrt{\frac{p_{n}}{n}}\right\} \cap \Omega_{K}\right) \\
\leq \varepsilon+\frac{C_{7.1}^{\prime}}{\sqrt{n p_{n}}} .
\end{array}
$$


(ii) Additionally, if

$$
\log \left(1 / p_{n}\right)<\bar{c}_{7.1}\left(\log n p_{n}\right)^{2}
$$

then

$$
\begin{array}{r}
\mathbb{P}\left(\left\{s_{\min }\left(A_{n}+D_{n}\right) \leq c_{7.1} \varepsilon \exp \left(-C_{7.1} \frac{\log \left(1 / p_{n}\right)}{\log \left(n p_{n}\right)}\right) \sqrt{\frac{p_{n}}{n}}\right\} \bigcap \Omega_{K}\right) \\
\leq \varepsilon+\exp \left(-c_{7.1}^{\prime} \sqrt{n p_{n}}\right) .
\end{array}
$$

The proof of part (i) of Theorem 7.1 follows from Berry-Esséen theorem and Proposition 3.4. The proof of part (ii) uses results from Section 5 and Section 6. Recall that in Section 5 and Section 6 we have shown that there does not exist any vector in $\operatorname{Ker}\left(B^{D}\right)$ with a sub-exponential two-dimensional LCD, with high probability. To prove the second part of Theorem 7.1, we use LCD based bounds on Lévy concentration function. At this moment, we know that with high probability, any vector in in $\operatorname{Ker}\left(B^{D}\right)$ has an exponential two-dimensional LCD. However, we do not have any control the real-imaginary de-correlation of this vector. This means that we cannot use the bound (5.7), and have to rely on (5.8).

To apply (5.8), we therefore need to show that any vector with a large twodimensional LCD must also admit a large value of one-dimensional LCD. This calls for another modification to the definition of the one-dimensional LCD.

Definition 7.2. For a non-zero vector $x \in \mathbb{R}^{m}$, we set

$$
\widehat{D}_{1}(x):=\inf \left\{\theta>0: \operatorname{dist}\left(\theta x, \mathbb{Z}^{m}\right)<L \sqrt{\log _{1} \frac{\|\theta x\|_{2}}{2^{8} L}}\right\} .
$$

The advantage of working with this one-dimensional LCD $\widehat{D}_{1}(\cdot)$ can be seen from the following result.

Lemma 7.3. For $z:=x+\mathrm{i} y \in \mathbb{C}^{m}$ we have $\widehat{D}_{1}(x) \geq D_{2}(z)$.

Proof. The proof follows by simply noting that if there exists a $\theta^{\prime}>0$ such that

$$
\operatorname{dist}\left(\theta^{\prime} x, \mathbb{Z}^{m}\right)<L \sqrt{\log _{1} \frac{\left\|\theta^{\prime} x\right\|_{2}}{2^{8} L}}
$$

then for $\theta=\left(\theta^{\prime}, 0\right)$ we also have that

$$
\operatorname{dist}\left(V^{\top} \theta, \mathbb{Z}^{m}\right)<L \sqrt{\log _{1} \frac{\left\|V^{\top} \theta\right\|_{2}}{2^{8} L}} .
$$

Now we are ready to prove Theorem 7.1. 
Proof of Theorem 7.1. The proof is similar to that of [9, Theorem 1.1]. We include it for completeness. Note that for any $\vartheta>0$,

$$
\begin{aligned}
& \mathbb{P}\left(\left\{s_{\min }\left(A_{n}+D_{n}\right) \leq \vartheta\right\} \cap \Omega_{K}\right) \\
\leq & \mathbb{P}\left(\left\{\inf _{x \in V^{c}}\left\|\left(A_{n}+D_{n}\right) x\right\|_{2} \leq \vartheta\right\} \cap \Omega_{K}\right)+\mathbb{P}\left(\left\{\inf _{x \in V}\left\|\left(A_{n}+D_{n}\right) x\right\|_{2} \leq \vartheta\right\} \cap \Omega_{K}\right),
\end{aligned}
$$

where

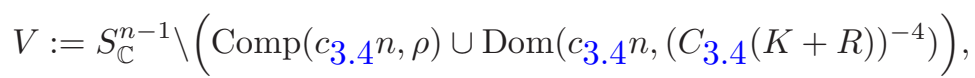

and $\rho$ as in Proposition 3.4. Using Proposition 3.4 with $M=c_{3.4}$, we obtain that

$\mathbb{P}\left(\inf _{x \in V^{c}}\left\|\left(A_{n}+D_{n}\right) x\right\|_{2} \leq c_{3.4}^{\prime}(K+R) \rho \sqrt{n p},\left\|A_{n}\right\| \leq K \sqrt{p n}\right) \leq \exp \left(-\bar{c}_{3.4^{n p}}\right)$.

Therefore it only remains to find an upper bound on the second term in the RHS of (7.2). Applying Lemma 2.7 we see that to find an upper bound of

$$
\mathbb{P}\left(\left\{\inf _{x \in V}\left\|\left(A_{n}+D_{n}\right) x\right\|_{2} \leq \varepsilon \rho^{2} \sqrt{\frac{p}{n}}\right\} \cap \Omega_{K}\right)
$$

is enough to find the same for

$$
\mathbb{P}\left(\left\{\operatorname{dist}\left(A_{n, j}, H_{n, j}\right) \leq \rho \sqrt{p} \varepsilon\right\} \cap \Omega_{K}\right) \text { for a fixed } j,
$$

where $A_{n, j}$ are columns of $\left(A_{n}+D_{n}\right)$. As these estimates are the same for different $j$ 's we only need to consider the case $j=1$. Recall that $B^{D}$ is the matrix whose rows are the columns $A_{n, 2}, \ldots, A_{n, n}$. Therefore

$$
\operatorname{dist}\left(A_{n, 1}, H_{n, 1}\right) \geq\left|\left\langle v, A_{n, 1}\right\rangle\right|,
$$

for any $v \in S_{\mathbb{C}}^{n-1} \cap \operatorname{Ker}\left(B^{D}\right)$. Thus it is enough to find an upper bound on

$$
\mathbb{P}\left(\left\{\exists v \in \mathcal{Z} \cap \operatorname{Ker}\left(B^{D}\right):\left|\left\langle A_{n, 1}, v\right\rangle\right| \leq \rho \varepsilon \sqrt{p}\right\} \cap \Omega_{K}\right) .
$$

First we obtain a bound on (7.3) under the assumption of part (i). This follows from a simple Berry-Esséen bound.

Since $v \in S_{\mathbb{C}}^{n-1} \cap \operatorname{Ker}\left(B^{D}\right)$ using Proposition 3.4 again, we may assume that

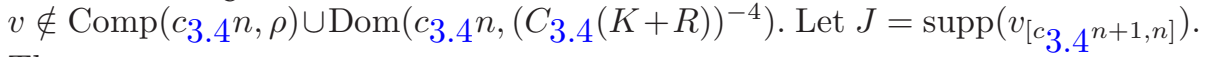
Then

$$
\mathbb{P}\left(\left|\left\langle A_{n, 1}, v\right\rangle\right| \leq \rho \varepsilon \sqrt{p}\right) \leq \mathcal{L}\left(\sum_{i \in J} v_{i} \delta_{i} \xi_{i}, \rho \sqrt{p} \varepsilon\right) .
$$

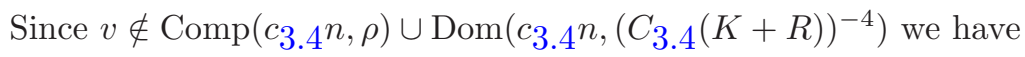

$$
\left\|v_{J}\right\|_{\infty} \leq \frac{\left.C_{3.4}(K+R)\right)^{4}}{\sqrt{c_{3.4^{n}}}}\left\|v_{J}\right\|_{2} \quad \text { and } \quad\left\|v_{J}\right\|_{2} \geq \rho .
$$


The Berry-Esséen Theorem (see [35, Theorem 2.2.17]) then yields that

$\mathcal{L}\left(\sum_{i \in J} v_{i} \delta_{i} \xi_{i}, \rho \sqrt{p} \varepsilon\right) \leq C \varepsilon+C^{\prime} \frac{p\left\|v_{J}\right\|_{3}^{3}}{p^{3 / 2}\left\|v_{J}\right\|_{2}^{3}} \leq C \varepsilon+C^{\prime} \frac{\left\|v_{J}\right\|_{\infty}}{p^{1 / 2}\left\|v_{J}\right\|_{2}} \leq C \varepsilon+\frac{C^{\prime \prime}}{\sqrt{p n}}$

where $C$ is an absolute constant, the constant $C^{\prime}$ depends only on the fourth moment of $\left\{\xi_{i, j}\right\}$, and

$$
C^{\prime \prime}=\frac{\left.C_{3.4}(K+R)\right)^{4}}{\sqrt{c_{3.4}}} \cdot C^{\prime}
$$

Replacing $\varepsilon$ by $\varepsilon / C$ finishes the proof of part (i) of the theorem.

It remains to prove part (ii). As seen above, we only need to obtain a bound on (7.3) under the stronger assumption of $p_{n}$ of part (ii). To this end, we apply Proposition 3.4 again. Setting $M_{0}=C_{3.1} \rho^{-4} p^{-1}$ from Proposition 3.4 we find that it is enough to bound

$$
\mathbb{P}\left(\left\{\exists v \in V_{0} \cap \operatorname{Ker}\left(B^{D}\right):\left|\left\langle A_{n, 1}, v\right\rangle\right| \leq \rho \varepsilon \sqrt{p}\right\} \cap \Omega_{K}\right),
$$

where

$$
V_{0}:=S_{\mathbb{C}}^{n-1} \backslash\left(\operatorname{Comp}\left(M_{0}, \rho\right) \cup \operatorname{Dom}\left(M_{0},\left(C_{3.4}(K+R)\right)^{-4}\right)\right) .
$$

Further denote

$$
V_{1}:=\left\{w \in V_{0}: D_{2}\left(w_{\text {small }} /\left\|w_{\text {small }}\right\|_{2}\right) \leq \exp \left(c^{\prime} n / M_{0}\right)\right\} \quad \text { and } \quad V_{2}:=V_{0} \backslash V_{1},
$$

where $c^{\prime}:=\min \left\{c_{5.1}^{\prime}, c_{6.4}^{\prime}\right\}$. We will show that

$$
\mathbb{P}\left(\left\{\exists v \in V_{1} \cap \operatorname{Ker}\left(B^{D}\right)\right\} \cap \Omega_{K}\right) \leq \exp (-\bar{c} n p),
$$

for some $\bar{c}>0$. Since $\operatorname{Ker}\left(B^{D}\right)$ is invariant under rotation, recalling the definition of the set $\mathcal{Z}$ (see (4.4)), we see that it is enough to show that

$$
\begin{aligned}
\mathbb{P}\left(\left\{\exists v \in \mathcal{Z} \cap \operatorname{Ker}\left(B^{D}\right): D_{2}\left(v_{\text {small }} /\left\|v_{\text {small }}\right\|_{2}\right) \leq \exp \left(c^{\prime} n / M_{0}\right)\right\}\right. & \left.\cap \Omega_{K}\right) \\
& \leq \exp (-\bar{c} n p) .
\end{aligned}
$$

Note that, if $p$ satisfies (7.1) with a sufficiently small $\bar{c}_{7.1}$, then it also satisfies the assumption (5.2). So we can apply Theorem 5.1. Applying Theorem 5.1 and Proposition 6.4 we then immediately obtain our claim (7.6). Therefore it only remains to find an upper bound on

$$
\begin{array}{r}
\mathbb{P}\left(\left\{\exists v \in \mathcal{Z} \cap \operatorname{Ker}\left(B^{D}\right): D_{2}\left(v_{\text {small }} /\left\|v_{\text {small }}\right\|_{2}\right)>\exp \left(c^{\prime} n / M_{0}\right)\right.\right. \text { and } \\
\left.\left.\left|\left\langle A_{n, 1}, v\right\rangle\right| \leq \rho \varepsilon \sqrt{p}\right\} \cap \Omega_{K}\right) .
\end{array}
$$


To obtain the desired bound we condition on $B^{D}$ which fixes the vector $v$ for which

$$
D_{2}\left(v_{\text {small }} /\left\|v_{\text {small }}\right\|_{2}\right)>\exp \left(c^{\prime} n / M_{0}\right) .
$$

Lemma 7.3 implies that

$$
\widehat{D}_{1}(\phi(v))>\exp \left(c^{\prime} n / M_{0}\right),
$$

where we recall that $v_{\text {small }} /\left\|v_{\text {small }}\right\|_{2}=\phi(v)+\mathrm{i} \psi(v)$. Inequality (5.8) holds with $\widehat{D}_{1}(\cdot)$ instead of $D_{1}(\cdot)$ if the constant $\bar{C}_{5.5}$ is appropriately adjusted. Recalling the definition of $M_{0}$ we deduce that

$\mathbb{P}\left(\left|\left\langle A_{n, 1}, v\right\rangle\right| \leq \varepsilon \rho \sqrt{p}\right) \leq \bar{C}\left(\varepsilon+\frac{1}{\sqrt{p} \widehat{D}_{1}(\phi(v))}\right) \leq \bar{C}\left(\varepsilon+\frac{1}{\sqrt{p}} \exp \left(-c^{\prime \prime} n p \rho^{4}\right)\right)$,

for some constants $\bar{C}$ and $c^{\prime \prime}$. Choosing $\bar{c}_{7.1}$ sufficiently small and recalling the definition of $\rho$ we further deduce that

$$
\frac{1}{\sqrt{p}} \exp \left(-c^{\prime \prime} n p \rho^{4}\right) \leq \exp \left(-c^{\prime \prime} \sqrt{n p}\right) .
$$

Therefore replacing $\varepsilon$ by $\varepsilon / \bar{C}$ we conclude that (7.7) is bounded by

$$
\varepsilon+\bar{C} \exp \left(-c^{\prime \prime} \sqrt{n p}\right)
$$

This completes the proof of the theorem.

Proof of Theorem 2.2. The proof immediately follows from Theorem 7.1, [9, Theorem 1.7], and the triangle inequality.

Remark 7.4. From the proof of Theorem 7.1 we note that the assumption (1.2) (equivalently (7.1)) was needed to show that the assumption (5.2) holds. From [9, Proposition 3.1] we have $\rho=\exp (-C \log (1 / p) / \log (n p))$, for some large $C$. If one can improve the conclusion of [9, Proposition 3.1] to accommodate $\rho=\Omega(1)$ then it is obvious that (5.2) holds without the assumption (1.2), and therefore Theorem 1.3(ii) can be extended without any extra assumption.

\section{Intermediate Singular Values}

The goal of this short section is to prove Theorem 2.10 which shows that there are not too many singular values of the matrix $\frac{1}{\sqrt{n p}} A_{n}-w I_{n}$ near zero. In proving Theorem 2.10, we employ the same strategy as in [13, 43, 47]. Namely, we first show that the distance of any row of $A_{n}$ from any given subspace of not very large dimension cannot be too small with large probability.

Lemma 8.1. Let $\boldsymbol{a}:=\left(\xi_{i} \delta_{i}\right)_{i=1}^{n}$ be an $n$-dimensional vector where $\left\{\xi_{i}\right\}_{i=1}^{n}$ are i.i.d. with zero mean and unit variance and $\{\delta\}_{i=1}^{n}$ are i.i.d. $\operatorname{Ber}(p)$. Let $\psi$ : $\mathbb{N} \mapsto \mathbb{N}$ be such that $\psi(n) \rightarrow \infty$ and $\psi(n)<n$. Then there exists a positive 
finite constant $c_{8.1^{2}}$ such that for every sub-space $H$ of $\mathbb{C}^{n}$ with $1 \leq \operatorname{dim}(H) \leq$ $n-\psi(n)$, we have

$\mathbb{P}\left(\operatorname{dist}(\boldsymbol{a}, H) \leq c_{8.1} \sqrt{p(n-\operatorname{dim}(H))}\right) \leq \exp \left(-c_{8.1} p \psi(n)\right)+\exp \left(-c_{8.1} \psi^{2}(n) / n\right)$.

A result similar to Lemma 8.1 was obtained in [43] (see Proposition 5.1 there) for the dense case. Later in [13] (and [47]) it was improved for the sparse case. Our Lemma 8.1 follows from [13, Lemma 3.5] when applied to the set-up of this paper. So we omit the proof and refer the reader to the proof of [13, Lemma 3.5].

We now complete the proof of Theorem 2.10 using Lemma 8.1. We use same approach as in [43, pp. 2055-2056] (see also the proof of [13, Lemma 3.14]).

Proof of Theorem 2.10. To lighten the notation, let us denote by $s_{1} \geq s_{2} \geq$ $\cdots \geq s_{n}$ the singular values of $\left(A_{n}-\sqrt{n p} w I_{n}\right)$. Fix $i$ such that $3 \psi(n) \leq i \leq$ $n-1$ and denote by $A_{n}^{m, w}$ the sub-matrix formed by first $m$ rows of the matrix $\left(A_{n}-\sqrt{n p} w I_{n}\right)$, where $m=n-\lceil i / 2\rceil$. Further denote by $s_{1}^{\prime} \geq s_{2}^{\prime} \geq \cdots \geq s_{m}^{\prime}$ the singular values of $A_{n}^{m, w}$. Using Cauchy's interlacing inequality we see that

$$
s_{n-i}^{\prime} \leq s_{n-i} .
$$

Next from [43, Lemma A.4] it follows that

$$
s_{1}^{\prime-2}+s_{2}^{\prime-2}+\cdots+s_{m}^{\prime-2}=\operatorname{dist}_{1}^{\prime-2}+\operatorname{dist}_{2}^{\prime-2}+\cdots+\operatorname{dist}_{m}^{\prime-2},
$$

where $\operatorname{dist}_{j}^{\prime}:=\operatorname{dist}\left(\boldsymbol{a}_{j}-w \sqrt{n p} e_{j}, H_{j, n}^{m, w}\right), \boldsymbol{a}_{j}^{\top}$ is the $j$-th row of the matrix $A_{n}, H_{j, n}^{m, w}$ is the subspace spanned by all the rows of $A_{n}^{m, w}$ except the $j$-th row, and $e_{j}$ is the $j$-th canonical basis. We also note that $\operatorname{dist}_{j} \leq \operatorname{dist}_{j}^{\prime}$, where $\operatorname{dist}_{j}:=\operatorname{dist}\left(\boldsymbol{a}_{j}, \operatorname{span}\left(H_{j, n}^{m, w}, e_{j}\right)\right)$. Thus from (8.1)-(8.2) we deduce

$$
\frac{i}{2 n} s_{n-i}^{-2} \leq \frac{1}{n} \sum_{j=n-i}^{m} s_{j}^{\prime-2} \leq \frac{1}{n} \sum_{j=1}^{m} \operatorname{dist}_{j}^{-2} .
$$

It is easy to note that $\operatorname{dim}\left(\operatorname{span}\left(H_{j, n}^{m, w}, e_{j}\right)\right) \leq m+1 \leq n-\psi(n)$ for all $j=$ $1,2, \ldots, m$. Therefore from Lemma 8.1 we further obtain

$$
\mathbb{P}\left(\text { dist }_{j} \leq c_{8.1} \sqrt{p \cdot i / 3}\right) \leq 2 n^{-4}, \quad j=1,2, \ldots, m,
$$

where we used the fact that $n-\operatorname{dim}\left(\operatorname{span}\left(H_{j, n}^{m, w}, e_{j}\right)\right) \geq n-(m+1) \geq i / 3$ and chose $C_{2.10} \geq 4 c_{8.1}^{-1}$. Hence, from (8.3) we see that

$$
\mathbb{P}\left(s_{n-i} \leq \frac{c_{8.1}}{\sqrt{3}} \cdot \sqrt{n p} \cdot \frac{i}{n}\right) \leq 2 n^{-3},
$$

for all $i$ such that $3 \psi(n) \leq i \leq n-1$. After taking the union over $i$, the proof of the theorem completes.

\footnotetext{
${ }^{2}$ the constant $c_{8.1}$ may depend on the tail of the distribution of $\left\{\xi_{i}\right\}_{i=1}^{n}$
} 


\section{Weak Convergence}

Here our goal is to prove Theorem 2.11. As mentioned in Section 2.3, using a truncation argument, we first show that it is enough to restrict to the case of bounded $\left\{\xi_{i, j}\right\}_{i, j=1}^{n}$. To this end, we have the following lemma.

Lemma 9.1. If the conclusion of Theorem 2.11 holds for $\left\{\xi_{i, j}\right\}_{i, j=1}^{n}$ bounded then it continues to hold without the boundedness assumption.

The proof of the truncation argument has now become standard in the random matrix literature which follows from an application of Hoffman-Wielandt inequality (see [3, Lemma 2.1.19]) upon using the fact that the bounded Lipschitz metric on the space of all probability measures on $\mathbb{R}$ metrizes the weak convergence of probability measures (see [3, Theorem C.8]). We refer the reader to [3, Appendix C.2] for a definition of the bounded Lipschitz metric. Using the above two ingredients and proceeding similarly as in the the proof of $[16$, Proposition 4.1] one can complete the proof of Lemma 9.1. Further details are omitted.

Equipped with Lemma 9.1, hereafter we may and will assume that $\left\{\xi_{i, j}\right\}_{i, j=1}^{n}$ are bounded by some constant $\mathfrak{K}_{0}$. It is well known that the conclusion of Theorem 2.11 holds for $\nu_{G_{n}}^{w}$, the symmetrized version of the empirical law of the singular values of $\left(n^{-1 / 2} G_{n}-w I_{n}\right)$, where $G_{n}$ is a real Ginibre matrix. Thus to prove Theorem 2.11 it is enough to show that

$$
\int f(x) d \nu_{G_{n}}^{w}(x)-\int f(x) d \nu_{n}^{w}(x) \rightarrow 0, \quad \text { as } n \rightarrow \infty, \quad \text { almost surely, }
$$

for every $f \in C_{c}(\mathbb{R})$, the set of all continuous and compactly supported functions on $\mathbb{R}$, and $w \in \mathbb{C}$. To prove (9.1) we first show that both the random probability measures $\nu_{n}^{w}$ and $\nu_{G_{n}}^{w}$ are close to their expectations, $\mathbb{E} \nu_{n}^{w}$ and $\mathbb{E} \nu_{G_{n}}^{w}$, respectively, and then we establish that $\mathbb{E} \nu_{n}^{w}$ and $\mathbb{E} \nu_{G_{n}}^{w}$ are close to each other as well. To carry out the first step we use the following concentration inequality.

Lemma 9.2. Let $H_{n}:=\left(h_{i, j}\right)_{i, j=1}^{n}$ be a Hermitian random matrix with entries on and above the diagonal are jointly independent. Let $f: \mathbb{R} \mapsto \mathbb{R}$ be an L-Lipschitz function supported on a compact interval $I \subset \mathbb{R}$, with $\|f\|_{\infty}:=$ $\sup _{x \in I}|f(x)| \leq 1$. Further let $H_{0}$ be an arbitrary $n \times n$ deterministic matrix and denote $\mathrm{H}_{n}:=H_{n}+H_{0}$. Fix an $\varepsilon>0$.

(i) If the entries of $H_{n}$ are uniformly bounded by $\mathfrak{K} / \sqrt{n}$ for some $\mathfrak{K}<\infty$ then

$\mathbb{P}\left(\left|\int f(x) d L_{\mathrm{H}_{n}}(x)-\mathbb{E} \int f(x) d L_{\mathrm{H}_{n}}(x)\right| \geq \varepsilon\right) \leq \frac{C_{9.2} L|I|}{\varepsilon} \exp \left(-\frac{c_{9.2^{n^{2}} \varepsilon^{4}}}{\mathfrak{K}^{2} L^{4}|I|^{2}}\right)$,

for some absolute constants $c_{9.2}, C_{9.2}>0$.

(ii) If the entries of $H_{n}$ satisfy the logarithmic Sobolev inequality with uniform constant $\mathfrak{L}$ then

$\mathbb{P}\left(\left|\int f(x) d L_{\mathrm{H}_{n}}(x)-\mathbb{E} \int f(x) d L_{\mathrm{H}_{n}}(x)\right| \geq \varepsilon\right) \leq 2 \exp \left(-\frac{c_{9.2} n^{2} \varepsilon^{2}}{8 \mathfrak{L} L^{2}}\right)$. 
Proof. Part (i) is a consequence of [19, Lemma 3.2]. For $H_{0}=0$, the proof part (ii) is immediate from [30, Theorem 1.1(b)]. A key step in the proof [30, Theorem 1.1(b)] is to show that for any Lipschitz function $f: \mathbb{R} \mapsto \mathbb{R}$ the map $H \mapsto \int f(x) d_{L_{H}}(x)$ is also a Lipschitz function (see [30, Lemma 1.2(b)]). The same proof shows that for any deterministic $H_{0}$ the map $H \mapsto \int f(x) d_{L_{H+H_{0}}}(x)$ is again Lipschitz. Therefore the general case follows.

Next we need to show that $\mathbb{E} \nu_{n}^{w}$ and $\mathbb{E} \nu_{G_{n}}^{w}$ are close to each other. This will be obtained upon showing that the corresponding Stieltjes transforms asymptotically are the same. Before stating the relevant result let us define the Stieltjes transform of a probability measure on $\mathbb{R}$.

Definition 9.3. Let $\mu$ be a probability measure on $\mathbb{R}$. Its Stieltjes transform is given by

$$
G_{\mu}(\zeta):=\int_{\mathbb{R}} \frac{1}{x-\zeta} d \mu(x), \quad \zeta \in \mathbb{C} \backslash \mathbb{R} .
$$

We write $m_{n}(\zeta):=m_{n}(\zeta, w)$ and $m_{G_{n}}(\zeta):=m_{G_{n}}(\zeta, w)$ to denote the Stieltjes transform of $\nu_{n}^{w}$ and $\nu_{G_{n}}^{w}$, respectively. We now have the following lemma.

Lemma 9.4. Let $A_{n}$ be an $n \times n$ matrix with entries $a_{i, j}=\delta_{i, j} \cdot \xi_{i, j}$, where $\delta_{i, j}$ are i.i.d. Bernoulli random variables with $\mathbb{P}\left(\delta_{i, j}=1\right)=p$, and $\xi_{i, j}$ are centered i.i.d. random variables with unit variance bounded by $\mathfrak{K}_{0}$ for some $\mathfrak{K}_{0}$. Fix any $w \in \mathbb{C}$ and $\zeta \in \mathbb{C}^{+}$. Then

$$
\left|\mathbb{E} m_{n}(\zeta)-\mathbb{E} m_{G_{n}}(\zeta)\right| \leq \frac{C_{9.4}}{\sqrt{n p}(\operatorname{Im} \zeta)^{4}}\left(1+\frac{1}{(n \operatorname{Im} \zeta)^{2}}\right),
$$

for some constant $C_{9.4}$ depending only on $\mathfrak{K}_{0}$.

A version of Lemma 9.4 was proved in [20] when $A_{n}$ is replaced by $B_{n}$, a matrix of i.i.d. centered $\operatorname{Ber}(p)$ random variables. A crucial step in the proof of [20, Lemma 8.2] is the Lindeberg replacement strategy of [17]. The latter depends only the bounds on the first three moments of the entries of $B_{n}$. Since $\left\{\xi_{i, j}\right\}_{i, j=1}^{n}$ are uniformly bounded by $\mathfrak{K}_{0}$ one can obtain the bounds necessary to apply [17, Theorem 1.1, Corollary 1.2]. Hence, following the same lines of arguments as in [20, Lemma 8.2] one completes the proof of Lemma 9.4.

Equipped with all the required ingredients we now finish the proof of Theorem 2.11 .

Proof of Theorem 2.11. It is known that for any $w \in \mathbb{C}$ the random probability measure $\nu_{G_{n}}^{w}$ converges weakly to $\nu_{\infty}^{w}$, for some probability measure, almost surely. For example, see [4]. Therefore, it is enough show that

$$
\int f(x) d \nu_{n}^{w}(x)-\int f(x) d \nu_{G_{n}}^{w}(x) \rightarrow 0, \quad \text { almost surely, } \quad \text { as } n \rightarrow \infty,
$$

for every bounded continuous function $f: \mathbb{R} \mapsto \mathbb{R}$. Since $\left\{\xi_{i, j}\right\}_{i, j=1}^{n}$ are uniformly bounded, by Chernoff's bound and Borel-Cantelli lemma it follows that the sequence of probability measures $\left\{\nu_{n}^{w}\right\}_{n \in \mathbb{N}}$ are almost surely compactly supported, 
whenever $n p \geq C_{0} \log n$ for some large constant $C_{0}$. The strong law of large number further shows that $\left\{\nu_{G_{n}}^{w}\right\}_{n \in \mathbb{N}}$ are almost surely compactly supported. Hence, we only need to show that (9.2) holds for continuous and compactly supported functions $f$.

Now, given any $\delta>0$, and a continuous and compactly supported function $f$, one can construct a Lipschitz function $f_{\delta}$, such that $\left\|f-f_{\delta}\right\|_{\infty} \leq \delta$ with the Lipschitz constant of $f_{\delta}$ depending only on $f$ and $\delta$. Hence, it suffices to establish (9.2) only for compactly supported Lipschitz functions.

To this end, recalling that Gaussian random variables satisfy the logarithmic Sobolev inequality (see [29]), from Lemma 9.2 we deduce that

$$
\int f(x) d \mathfrak{u}_{n}(x)-\mathbb{E} \int f(x) d \mathfrak{u}_{n}(x) \rightarrow 0 \quad \text { as } n \rightarrow \infty,
$$

almost surely, for $\mathfrak{u}_{n}=\nu_{n}^{w}$ and $\nu_{G_{n}}^{w}$. Since $n p \rightarrow \infty$ as $n \rightarrow \infty$, from Lemma 9.4 it also follows that (one can argue similarly as in the proof of [3, Theorem 2.4.3])

$$
\mathbb{E} \int f(x) d \nu_{n}^{w}(x)-\mathbb{E} \int f(x) d \nu_{G_{n}}^{w}(x) \rightarrow 0, \quad \text { as } n \rightarrow \infty .
$$

Combining (9.3)-(9.4) we establish that (9.2) holds for any Lipschitz and compactly supported function $f$. This completes the proof.

\section{Proof of Theorem 1.3}

In this section we combine Theorem 2.2, Theorem 2.10, and Theorem 2.11 to prove Theorem 1.3. As already mentioned in Section 2, to prove Theorem 1.3 we need to invoke the replacement principle. We fix $r \in(0,1)$ and define $\mathbb{D}_{r}:=$ $\left\{w \in B_{\mathbb{C}}(0,1-r):|\operatorname{Im} w| \geq r\right\}$. Then applying Lemma 2.1, we show that for every $f \in C_{c}^{2}(\mathbb{C})$ supported on $\mathbb{D}_{r}$, we have $\int f(w) d L_{n}(w) \rightarrow \int f(w) d \mathfrak{m}(w)$ in probability or almost surely, depending on the choice of the sparsity parameter $p$, where $L_{n}$ is the ESD of $\frac{1}{\sqrt{n p}} A_{n}$. Afterwards letting $r \rightarrow 0$ we establish the circular law limit. Below we make this idea precise.

Before we prove Theorem 1.3, we need some properties of the probability measure $\nu_{\infty}^{w}$. Recall that $\nu_{\infty}^{w}$ is the limit of the ESD of $\boldsymbol{A}_{n}^{w}$ where $\boldsymbol{A}_{n}^{w}$ was defined in (2.7).

Lemma 10.1. (i) For any $w \in B_{\mathbb{C}}(0,1)$ the probability measure $\nu_{\infty}^{w}$ is supported on $\left[-\sqrt{\lambda_{+}}, \sqrt{\lambda_{+}}\right]$, where

$$
\lambda_{+}:=\lambda_{+}(w):=\frac{\left(\sqrt{1+8|w|^{2}}+3\right)^{3}}{8\left(\sqrt{1+8|w|^{2}}+1\right)} .
$$

(ii) There exists some absolute constant $r_{0} \in(0,1)$ such that for all $r \in\left(0, r_{0}\right)$, $\tau \in(0,1)$, and $w \in B_{\mathbb{C}}(0,1-r)$ we have

$$
\int_{-\tau}^{\tau}|\log | x|| d \nu_{\infty}^{w}(x) \leq C_{10.1} \tau|\log \tau|
$$


for some positive constant $C_{10.1}$ which depends only on $r$.

Proof. In [4, Lemma 4.2] it was shown that for any $w \in B_{\mathbb{C}}(0,1)$ the probability measure $\widetilde{\nu}_{\infty}^{w}$ is supported on $\left[0, \lambda_{+}(w)\right]$ where for any $t>0, \widetilde{\nu}_{\infty}^{w}\left(\left(0, t^{2}\right)\right)=$ $\nu_{\infty}^{w}((-t, t))$. From this part (i) of the lemma follows.

Turning to prove (ii), using integration by parts we note that for any probability measure $\mu$ on $\mathbb{R}$ and $0<\tau<1$,

$$
\int_{0}^{\tau}|\log (x)| d \mu(x) \leq|\log (\tau)| \mu((0, \tau))+\int_{0}^{\tau} \frac{\mu((0, t))}{t} d t .
$$

Using [7, Lemma 7.8] and [7, Lemma 8.3] we see that for any $t \in(0,1)$,

$$
\nu_{\infty}^{w}((0, t)) \leq \nu_{\infty}^{w}((-t, t)) \leq 2 t \cdot\left(\operatorname{Im} m_{\infty}(\mathrm{i} t)\right) \leq 2 C t,
$$

for some large constant $C$ depending on $r$. The rest follows from (10.1).

We are now ready to prove Theorem 1.3.

Proof of Theorem 1.3. We first prove part (i). That is we show that the ESD of $A_{n} / \sqrt{n p}$ converges weakly to the circular law, in probability. Fix $r \in(0,1 / 2)$ and denote $\mathbb{D}_{r}:=\left\{w \in B_{\mathbb{C}}(0,1-r):|\operatorname{Im} w| \geq r\right\}$. Let us also fix $w \in \mathbb{D}_{r}$. Define

$$
\Omega_{n}^{\prime}:=\left\{s_{\min }\left(\frac{A_{n}}{\sqrt{n p}}-w I_{n}\right) \geq c_{n}\right\}
$$

where

$$
c_{n}:=c_{2.2} \exp \left(-C_{2.2} \frac{\log (1 / p)}{\log (n p)}\right) \frac{1}{n \sqrt{n p}} .
$$

Setting $D_{n}:=-w \sqrt{n p} I_{n}$ and applying Theorem 2.2 we deduce that

$$
\mathbb{P}\left(\Omega_{n}^{\prime}\right) \geq 1-\frac{1+C_{2.2}^{\prime}}{\sqrt{n p}} .
$$

Fix any $\delta \in(0,1)$ and let $\tau:=\tau(\delta):=c_{2.10} \delta$. Further denote $\psi(n):=$ $\max \left\{\sqrt{n / p}, n /(\log n)^{2}\right\}$. Since $n p=\omega\left(\log ^{2} n\right)$ we note that $\psi(n)=o(n / \log n)$. Equipped with these notations we recall the definition of $\nu_{n}^{w}$ to see that

$$
\begin{aligned}
& \int_{-\tau}^{\tau}|\log (|x|)| d \nu_{n}^{w}(x) \\
= & \frac{1}{n} \sum_{i=1}^{n-3 \psi(n)}\left|\log \left(s_{i}\right)\right| \mathbb{I}\left(s_{i} \leq \tau\right)+\frac{1}{n} \sum_{i=n-3 \psi(n)+1}^{n}\left|\log \left(s_{i}\right)\right| \mathbb{I}\left(s_{i} \leq \tau\right) .
\end{aligned}
$$

We evaluate each term of the RHS of (10.3) separately. Focusing on the second term we see that on the event $\Omega_{n}^{\prime}$

$$
\frac{1}{n} \sum_{i=n-3 \psi(n)+1}^{n}\left|\log \left(s_{i}\right)\right| \mathbb{I}\left(s_{i} \leq \tau\right) \leq\left|\log \left(s_{n}\right)\right| \cdot \frac{3 \psi(n)}{n} \leq \log c_{n}^{-1} \cdot \frac{3 \psi(n)}{n}=o(1) .
$$


We next consider the first term of (10.3). Denote the event described in Theorem 2.10 by $\Omega_{n}^{\prime \prime}$. Since $\min \left\{p \psi(n), \psi^{2}(n) / n\right\} \geq C_{2.10} \log n$, on the event $\Omega_{n}^{\prime \prime}$ we have

$$
\begin{aligned}
& \frac{1}{n} \sum_{i=1}^{n-3 \psi(n)}\left|\log \left(s_{i}\right)\right| \mathbb{I}\left(s_{i} \leq \tau\right)=\frac{1}{n} \sum_{i=3 \psi(n)}^{n-1}\left|\log \left(s_{n-i}\right)\right| \mathbb{I}\left(s_{n-i} \leq \tau\right) \\
& \leq \frac{\log \left(1 / c_{2.10}\right)}{n} \sum_{i=3 \psi(n)}^{n-1} \mathbb{I}\left(s_{n-i} \leq \tau\right)+\frac{1}{n} \sum_{i=3 \psi(n)}^{n-1} \log \left(\frac{n}{i}\right) \mathbb{I}\left(s_{n-i} \leq \tau\right),
\end{aligned}
$$

and by Theorem 2.10, $\mathbb{P}\left(\left(\Omega_{n}^{\prime \prime}\right)^{c}\right) \leq 2 / n^{2}$. Recalling the definition of $\tau$, from Theorem 2.10 it also follows that

$$
s_{n-i} \leq \tau \Rightarrow i \leq \delta n
$$

on the event $\Omega_{n}^{\prime \prime}$. So from (10.5) we deduce that

$$
\begin{array}{r}
\frac{1}{n} \sum_{i=1}^{n-3 \psi(n)}\left|\log \left(s_{i}\right)\right| \mathbb{I}\left(s_{i} \leq \tau\right) \leq \delta \cdot \log \left(1 / c_{2.10}\right)+\frac{1}{n} \sum_{i=3 \psi(n)}^{\delta n} \log \left(\frac{n}{i}\right) \\
\leq \delta \cdot \log \left(1 / c_{2} .10\right)-2 \int_{0}^{\delta} \log x d x
\end{array}
$$

for all large $n$. Hence, denoting $\Omega_{n}:=\Omega_{n}^{\prime} \cup \Omega_{n}^{\prime \prime}$, from (10.3)-(10.4) and (10.6) we obtain that

$$
\int_{-\tau(\delta)}^{\tau(\delta)}|\log (|x|)| d \nu_{n}^{w}(x) \leq \kappa(\delta)
$$

for all large $n$, on the event $\Omega_{n}$, where $\kappa(\delta):=2 \delta \cdot \log \left(1 / c_{2.10}\right)-2 \int_{0}^{\delta} \log x d x$. Note that $\lim _{\delta \rightarrow 0} \kappa(\delta)=0$. Therefore given any $\kappa>0$ there exists $\tau_{\kappa}:=\tau(\kappa)$, with the property $\lim _{\kappa \rightarrow 0} \tau_{k}=0$, such that

$$
\begin{aligned}
\limsup _{n \rightarrow \infty} \mathbb{P}\left(\int_{-\tau_{\kappa}}^{\tau_{\kappa}}\right. & \left.|\log | x|| d \nu_{n}^{w}(x) \geq \kappa\right) \\
& \leq \limsup _{n \rightarrow \infty} \mathbb{P}\left(\left\{\int_{-\tau_{\kappa}}^{\tau_{\kappa}}|\log | x|| d \nu_{n}^{w}(x) \geq \kappa\right\} \cap \Omega_{n}\right)=0 .
\end{aligned}
$$

Next noting that $\log (\cdot)$ is a bounded function on a compact interval that is bounded away from zero, we apply Theorem 2.11 to deduce that

$$
\int_{\left(-R,-\tau_{\kappa}\right) \cup\left(\tau_{\kappa}, R\right)}|\log | x|| d \nu_{n}^{w}(x) \rightarrow \int_{\left(-R,-\tau_{\kappa}\right) \cup\left(\tau_{\kappa}, R\right)}|\log | x|| d \nu_{\infty}^{w}(x)
$$

in probability, for any $R \geq 1$. Recall that for $w \in \mathbb{D}_{r}$ the support of $\nu_{\infty}^{w}$ is contained in $[-6,6]$ (see Lemma 10.1(i)). On the other hand, from [9, Theorem 1.7] it follows that there exists a $K_{0}<\infty$ such that

$$
\mathbb{P}\left(\left\|A_{n}\right\| \geq K_{0} \sqrt{n p}\right) \leq \exp (-c n p)
$$


for some constant $c>0$. Therefore, using Borel-Cantelli lemma we deduce that

$$
\int_{\left(-R_{0}, R_{0}\right)^{c}}|\log | x|| d \nu_{n}^{w}(x) \rightarrow \int_{\left(-R_{0}, R_{0}\right)^{c}}|\log | x|| d \nu_{\infty}^{w}(x), \quad \text { almost surely, }
$$

for some $R_{0}>0$. From Lemma 10.1(ii) we also have that

$$
\int_{-\tau_{\kappa}}^{\tau_{\kappa}}|\log | x|| d \nu_{\infty}^{w}(x) \leq C \tau_{\kappa}\left|\log \tau_{\kappa}\right|
$$

for some constant $C$. As $\kappa>0$ is arbitrary and $\tau_{\kappa} \rightarrow 0$ as $\kappa \rightarrow 0$, combining (10.7)-(10.10) we deduce that

$$
\frac{1}{n} \log \left|\operatorname{det}\left(A_{n} / \sqrt{n p}-w I_{n}\right)\right|=\int_{-\infty}^{\infty} \log |x| d \nu_{n}^{w}(x) \rightarrow \int_{-\infty}^{\infty} \log |x| d \nu_{\infty}^{w}(x),
$$

in probability. Now the rest of the proof is completed using Lemma 2.1. Indeed, consider $\mathfrak{G}_{n}$ the $n \times n$ matrix with i.i.d. centered Gaussian entries with variance one. It is well-known that, for Lebesgue almost all $w$,

$$
\frac{1}{n} \log \left|\operatorname{det}\left(\mathfrak{G}_{n} / \sqrt{n}-w I_{n}\right)\right| \rightarrow \int_{-\infty}^{\infty} \log |x| d \nu_{\infty}^{w}(x), \text { almost surely. }
$$

For example, one can obtain a proof of (10.12) using [14, Lemma 4.11, Lemma 4.12], [15, Theorem 3.4], and [36, Lemma 3.3].

Thus setting $\mathbb{D}=\mathbb{D}_{r}, B_{n}^{(1)}=A_{n} / \sqrt{n p}$, and $B_{n}^{(2)}=\mathfrak{G}_{n} / \sqrt{n}$ in Lemma 2.1(a) we see that assumption (ii) there is satisfied. The assumption (i) of Lemma 2.1(a) follows from weak laws of large numbers for triangular arrays. Hence, using Lemma 2.1(i) and the Circular law for i.i.d. Gaussian matrix of unit variance (e.g. [43, Theorem 1.13]), we obtain that for every $r>0$ and every $f_{r} \in C_{c}^{2}(\mathbb{C})$, supported on $\mathbb{D}_{r}$,

$$
\int f_{r}(w) d L_{n}(w) \rightarrow \frac{1}{\pi} \int f_{r}(w) d \mathfrak{m}(w), \text { in probability. }
$$

To finish the proof it now remains to show that one can extend the convergence of (10.13) to all $f \in C_{c}^{2}(\mathbb{C})$. It follows from a standard argument.

Indeed, for any $r>0$ define $i_{r} \in C_{c}^{2}(\mathbb{C})$ such that $i_{r}$ is supported on $\mathbb{D}_{r}$ and $i_{r} \equiv 1$ on $\mathbb{D}_{2 r}$, and $i_{r}\left(\mathbb{D}_{r} \backslash \mathbb{D}_{2 r}\right) \subset[0,1]$. Fixing any $\delta>0$ choose an $r>0$ such that $r \leq \delta / 64 \mathfrak{K}$ where $\mathfrak{K}:=\|f\|_{\infty}:=\sup _{w \in \mathbb{C}}|f(w)|$. Denote $f_{r}:=f i_{r}$ and $\bar{f}_{r}:=f-f_{r}$. Applying (10.13) for the function $i_{r}$ and the triangle inequality we find that

$$
\begin{array}{r}
\mathbb{P}\left(\left|\int \bar{f}_{r}(w) d L_{n}(w)\right| \geq \delta / 4\right) \leq \mathbb{P}\left(\left|\int\left(1-i_{r}(w)\right) d L_{n}(w)\right| \geq \frac{\delta}{4 \mathfrak{K}}\right) \\
\leq \mathbb{P}\left(\left|\int i_{r}(w) d L_{n}(w)-\frac{1}{\pi} \int i_{r}(w) d \mathfrak{m}(w)\right| \geq \frac{\delta}{8 \mathfrak{K}}\right) \rightarrow 0
\end{array}
$$


as $n \rightarrow \infty$, where the last step follows from the fact that

$$
\left|\frac{1}{\pi} \int_{B_{\mathbb{C}}(0,1)}\left(1-i_{r}(w)\right) d \mathfrak{m}(w)\right| \leq \frac{1}{\pi} \int_{B_{\mathbb{C}}(0,1) \backslash \mathbb{D}_{2 r}} d \mathfrak{m}(w) \leq \frac{\delta}{8 \mathfrak{K}},
$$

by our choice of $r$. Thus combining (10.13)-(10.15) and the triangle inequality we find that for any $f \in C_{c}^{2}(\mathbb{C})$

$$
\begin{aligned}
\mathbb{P}\left(\left|f(w) d L_{n}(w)-\frac{1}{\pi} \int f(w) d \mathfrak{m}(w)\right|\right. & \geq \delta) \\
\leq \mathbb{P}\left(\left|f_{r}(w) d L_{n}(w)-\frac{1}{\pi} \int f_{r}(w) d \mathfrak{m}(w)\right| \geq \delta / 2\right) & +\mathbb{P}\left(\left|\int \bar{f}_{r}(w) d L_{n}(w)\right| \geq \delta / 4\right) \rightarrow 0,
\end{aligned}
$$

as $n \rightarrow \infty$. This completes the proof of the first part of the theorem. To prove the second part of the theorem we note that under the assumption (1.2), using Theorem 2.2 it follows

$$
\mathbb{P}\left(\tilde{\Omega}_{n}^{\prime}\right) \geq 1-O\left(\frac{1}{n^{2}}\right)
$$

where

$\tilde{\Omega}_{n}^{\prime}:=\left\{s_{\min }\left(\frac{A_{n}}{\sqrt{n p}}-w I_{n}\right) \geq \tilde{c}_{n}\right\}$, and $\tilde{c}_{n}:=c_{2.2} \exp \left(-C_{2.2} \frac{\log \left(1 / p_{n}\right)}{\log \left(n p_{n}\right)}\right) \cdot n^{-3}$.

Therefore, proceeding similarly as above, applying Borel-Cantelli lemma, and using Theorem 2.11(ii) we see that the conclusions of (10.7)-(10.8) hold almost surely.

Thus under the assumption (1.2) we have shown that (10.11) holds almost surely. Therefore proceeding same as above and using Lemma 2.1(ii) we obtain that for every $r>0$ and every $f \in C_{c}^{2}(\mathbb{C})$,

$$
\int f_{r}(w) d L_{n}(w) \rightarrow \frac{1}{\pi} \int f_{r}(w) d \mathfrak{m}(w), \text { almost surely, }
$$

where we recall that $f_{r}=f \cdot i_{r}$. The same proof shows that

$$
\limsup _{n \rightarrow \infty} \int\left(1-i_{r}(w)\right) d L_{n}(w) \leq \frac{1}{\pi} \int_{B_{\mathbb{C}}(0,1)}\left(1-i_{r}(w)\right) d \mathfrak{m}(w) \leq 8 r
$$

almost surely. Therefore,

$$
\limsup _{n \rightarrow \infty}\left|\int f(w) d L_{n}(w)-\frac{1}{\pi} \int_{B_{\mathbb{C}}(0,1)} f(w) d \mathfrak{m}(w)\right| \leq 16 \mathfrak{K} r \text {, almost surely, }
$$

where we recall $\mathfrak{K}=\|f\|_{\infty}$. Since $r$ is arbitrary the result follows. 


\section{Acknowledgements}

We thank the anonymous referee for her/his comments that lead to an improvement of the presentation and shortening of the paper. A.B. thanks Amir Dembo and Ofer Zeitouni for suggesting the problem and helpful conversations.

\section{References}

[1] R. Adamczak. On the Marchenko-Pastur and circular laws for some classes of random matrices with dependent entries. Electronic Journal of Probabability, 16(37), 1068-1095, 2011.

[2] R. Adamczak, D. Chafaï, and P. Wolff. Circular law for random matrices with exchangeable entries. Random Structures 83 Algorithms, 48, 454-479, 2016.

[3] G. W. Anderson, A. Guionnet, and O. Zeitouni. An introduction to random matrices. No. 118. Cambridge University Press, 2010.

[4] Z. D. Bai. Circular law. The Annals of Probability, 25(1), 494-529, 1997.

[5] Z. D. Bai. Methodologies in spectral analysis of large dimensional random matrices, a review. Statistica Sinica, 9, 611-677, 1999.

[6] Z. D. Bai and J. W. Silverstein. Spectral analysis of large dimensional random matrices, volume 20. Springer, 2010.

[7] A. Basak, N. Cook, and O. Zeitouni. Circular law for the sum of random permutation matrices. Electronic Journal of Probability, 23, paper no. 33, 51 pp., 2018.

[8] A. Basak and M. Rudelson. The circular law for sparse nonHermitian matrices. Extended version. ArXiv preprint, 2017. Available at https://arxiv.org/abs/1707.03675v1.

[9] A. Basak and M. Rudelson. Invertibility of sparse non-Hermitian matrices. Advances in Mathematics, 310, 426-483, 2017.

[10] A. Basak and M. Rudelson. The local circular law for sparse non-Hermitian matrices. In prepration, 2017.

[11] F. Benaych-Georges and A. Knowles. Lectures on the local semicircle law for Wigner matrices. To appear in SMF volume Panoramas et Synthéses, 2016.

[12] C. Bordenave, P. Caputo, and D. Chafaï. Circular law theorem for random Markov matrices. Probability Theory and Related Fields, 152(3-4), 751-779, 2012.

[13] C. Bordenave, P. Caputo, and D. Chafaï. Spectrum of Markov generators on sparse random graphs. Communications in Pure and Applied Mathematics, 67(4), 621-669, 2014.

[14] C. Bordenave and D. Chafaï. Around the circular law. Probability Surveys, $9(0), 1-89,2012$.

[15] P. Bourgade, H.-T. Yau, and J. Yin. Local circular law for random matrices. Probability Theory and Related Fields, 159(3), 545-595, 2014.

[16] W. Bryc, A. Dembo, and T. Jiang. Spectral measure of large random 
Hankel, Markov and Toeplitz matrices. Annals of Probability, 34(1), 1-38, 2006.

[17] S. Chatterjee. A simple invariance theorem. Preprint, 2005. Available at https://arxiv.org/abs/0508.213.

[18] S. Chatterjee. A generalization of the Lindeberg principle. The Annals of probability, 34(6), 2061-2076, 2006.

[19] N. Cook. The circular law for random regular digraphs with random edge weights. Random Matrices: Theory and Applications, 06, 1750012, 2017.

[20] N. Cook. The circular law for random regular digraphs. Preprint, 2017. Available at https://arxiv.org/abs/1703.05839.

[21] R. Durrett, Rick. Probability: theory and examples. Cambridge university press, 2010.

[22] A. Edelman. Eigenvalues and condition numbers of random matrices. SIAM Journal on Matrix Analysis and Applications, 9(4), 543-560, 1988.

[23] L. Erdős, H.-T. Yau, and J. Yin. Bulk universality for generalized Wigner matrices. Probability Theory and Related Fields, 154, 341-407, 2012.

[24] C. G. Esséen. On the Kolmogorov-Rogozin inequality for the concentration function. Zeitschrift für Wahrscheinlichkeitstheorie und Verwandte Gebiete. 5(3),210-216, 1966.

[25] S. Ge. Eigenvalue spacing of i.i.d. random matrices. Preprint, 2017.

[26] J. Ginibre. Statistical ensembles of complex, quaternion, and real matrices. Journal of Mathematical Physics, 6, 440-449, 1965.

[27] V. L. Girko. Circular law. Teoriya Veroyatnostei i ee Primeneniya, 29(4), 669-679, 1984.

[28] F. Götze and A. Tikhomirov. The circular law for random matrices. Annals of Probability, 38 (4), 1444-1491, 2010.

[29] L. Gross. Logarithmic Sobolev inequalities. American Journal of Mathematics, 97(4), 1061-1083, 1975.

[30] A. Guionnet and O. Zeitouni. Concentration of the spectral measure for large matrices. Electronic Communications in Probability, 5, 119-136, 2000.

[31] A. Litvak, A. Lytova, K. Tikhomirov, N. Tomczak-Jaegermann, and P. Youssef. Circular law for sparse random regular digraphs. Preprint, 2018. Available at https://arxiv.org/abs/1801.05576.

[32] H. H. Nguyen. Random doubly stochastic matrices: the circular law. The Annals of Probability, 42(3), 1161-1196, 2014.

[33] H. H. Nguyen and V. Vu. Circular law for random discrete matrices of given row sum. Journal of Combinatorics, 4(1), 1-30, 2013.

[34] L. A. Pastur. On the spectrum of random matrices. Theoretical and Mathematical Physics, 10(1), 67-74, 1972.

[35] D. W.Stroock. Probability theory: An analytic view. Second edition. Cambridge University Press, Cambridge, 2011.

[36] M. Rudelson. Invertibility of random matrices: Norm of the inverse. Annals of Mathematics, 168, 575-600, 2008.

[37] M. Rudelson and R. Vershynin. The Littlewood-Offord Problem and invertibility of random matrices. Advances in Mathematics, 218 (2), 600-633, 2008. 
[38] M. Rudelson and R. Vershynin. Smallest singular value of a random rectangular matrix. Communications on Pure and Applied Mathematics, 62, 1707-1739, 2009.

[39] M. Rudelson and R. Vershynin. No-gaps delocalization for general random matrices. Geometric and Functional Analysis, 26(6), 1716-1776, 2016.

[40] M. Rudelson and O. Zeitouni. Singular values of Gaussian matrices and permanent estimators. Random Structures Algorithms 48(1), 183-212, 2016.

[41] T. Tao. Topics in random matrix theory. Vol. 132, American Mathematical Society, 2012.

[42] T. Tao and V. Vu. Random matrices: the circular law. Communications in Contemporary Mathematics, 10(2), 261-307, 2008.

[43] T. Tao and V. Vu. Random matrices: universality of the ESDs and the circular law. Annals of Probability, 38, 2023-2065, 2010 (with an appendix by M. Krishnapur).

[44] R. Vershynin. Invertibility of symmetric random matrices. Random Structures \& Algorithms, 44(2), 135-182, 2014.

[45] E. P. Wigner. Characteristic vectors of bordered matrices. Annals of Mathematics, 62(3), 548-564, 1955.

[46] E. P. Wigner. On the distribution of the roots of certain symmetric matrices. Annals of Mathematics, 67(2), 325-327, 1958.

[47] P. M. Wood. Universality and the circular law for sparse random matrices. Annals of Applied Probability, 22(3), 1266-1300, 2012. 